\title{
A Computational Tool for the Rapid Design and Prototyping of Propellers for Underwater Vehicles
}

\author{
by \\ Kathryn Port D’Epagnier \\ B.S. Ocean Engineering \\ The United States Naval Academy (2005) \\ Submitted to the Department of Mechanical Engineering \\ in Partial Fulfillment of the Requirements for the Degree of \\ Master of Science in Mechanical Engineering \\ at the \\ Massachusetts Institute of Technology \\ and the \\ Woods Hole Oceanographic Institution
}

September 2007

(C2007 Kathryn Port D’Epagnier. All rights reserved.

The author hereby grants to MIT/WHOI permission to reproduce and to distribute publicly paper and electronic copies of this thesis document in whole or in part in any medium now known or hereafter created.

Signature of Author

Department of Mechanical Engineering

Aug 24, 2007

Certified by

Patrick J. Keenan

Professor of the Practice of Naval Architecture

Thesis Supervisor

Certified by

Richard W. Kimball

Thesis Supervisor

Accepted by

Lallit Anand

Professor of Mechanical Engineering Chairman, Departmental Committee on Graduate Students 
This page intentionally left blank. 


\section{A Computational Tool for the Rapid Design and Prototyping of Propellers for Underwater Vehicles \\ by}

Kathryn Port D'Epagnier

Submitted to the Department of Mechanical Engineering on August 24, 2007

in Partial Fulfillment of the Requirements for the Degree of

Master of Science in Mechanical Engineering

\section{Abstract}

An open source, MATLAB ${ }^{\text {TM }}$-based propeller design code MPVL was improved to include rapid prototyping capabilities as well as other upgrades as part of this effort. The resulting code, OpenPVL is described in this thesis. In addition, results from the development code BasicPVL are presented. An intermediate code, BasicPVL, was created by the author while OpenPVL was under development, and it provides guidance for initial propeller designs and propeller efficiency analysis. OpenPVL is part of the open source software suite of propeller design codes, OpenProp. OpenPVL is in the form of a Graphical User Interface (GUI) which features both a parametric design technique and a single propeller geometry generator. This code combines a user-friendly interface with a highly modifiable platform for advanced users. This tool offers graphical propeller design feedback while recording propeller input, output, geometry, and performance. OpenPVL features the ability to translate the propeller design geometry into a file readable by a Computer Aided Design (CAD) program and converted into a 3D-printable file.

Efficient propellers reduce the overall power requirements for Autonomous Underwater Vehicles (AUVs), and other propulsion-powered vehicles. The focus of this study is based on the need of propeller users to have an open source computer-based engineering tool for the rapid design of propellers suited to a wide range of underwater vehicles. Propeller vortex lattice lifting line (PVL) code in combination with 2D foil theory optimizes propeller design for AUVs. Several case studies demonstrate the functionality of OpenPVL, and serve as guides for future propeller designs. The first study analyzes propeller thruster performance characteristics for an off-the-shelf propeller, while the second study demonstrates the process for propeller optimization-from the initial design to the final file that can be read by a 3D printer. The third study reviews the complete process of the design and production of an AUV propeller. Thus, OpenPVL performs a variety of operations as a propeller lifting line code in streamlining the propeller optimization and prototyping process.

Thesis Supervisor: Patrick J. Keenan

Title: Professor of the Practice of Naval Architecture 


\section{Acknowledgements}

The author greatly appreciates the dedicated guidance of Richard W. Kimball, and is thankful for the time he has devoted to sharing his wealth of knowledge on the subject matter in this thesis.

The author expresses thanks to Rear Admiral David A. Gove, Oceanographer of the United States Navy, for academic funding and making this study possible. The author also would also like to thank CAPT P. Keenan, USN, for his support.

Additionally, the author would like to credit the Deep Submergence Lab at Woods Hole Oceanographic Institute, for use of their vehicles and resources in testing for this project.

Furthermore, the author is grateful for the other Navy and MIT/WHOI Joint Program students and the time spent studying and collaborating together. The author thanks the members of her propeller design group, for their contributions, and especially Hsin-Lung Chung for developing the foundations of the open source project OpenProp. The author would also like to thank Jordan Stanway, for providing information on automating RHINO ${ }^{\mathrm{TM}}$ scripting.

Finally, the author expresses deep gratitude towards her family and close friends for encouraging both her research and her US Navy career. 


\section{Table of Contents}

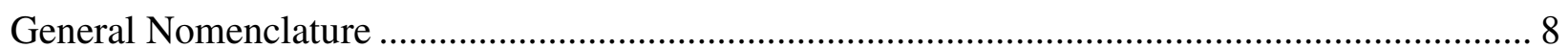

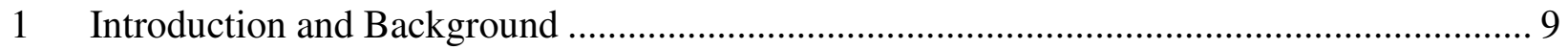

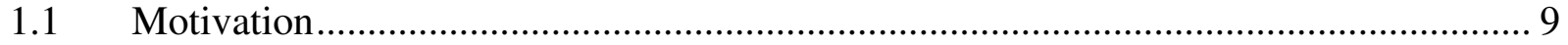

1.2 Recent Propeller Design Efforts ..................................................................... 10

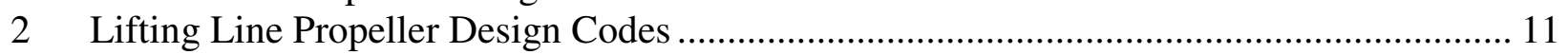

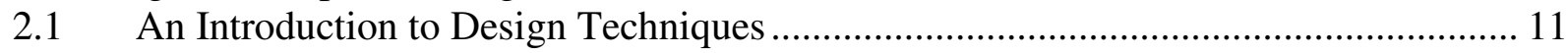

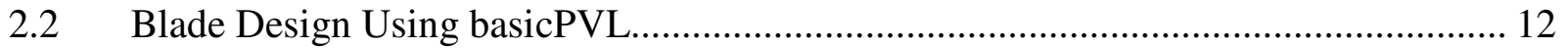

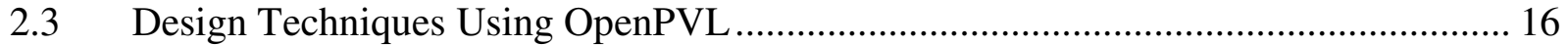

2.3.1 Parametric Analysis ........................................................................................ 17

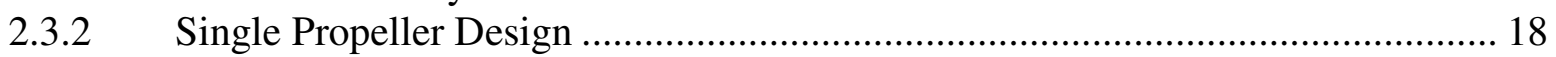

3 Propeller Printing: from Design Geometry to Prototype ………………………………...... 21

$3.1 \quad$ Blade Geometry from OpenPVL to .STL Files ........................................................ 21

3.2 Fusion Deposition Modeling (FDM) Printer Overview.............................................. 23

3.3 Analysis of Materials for Fusion Deposition Modeling ………………………......... 25

4 Studies on an Off-The-Shelf Propeller for the SeaBED class AUV ………........................ 28

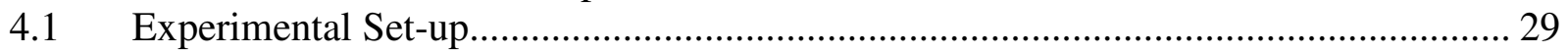

4.2 Method for Bollard and Mobile Cart Test Analysis ................................................... 31

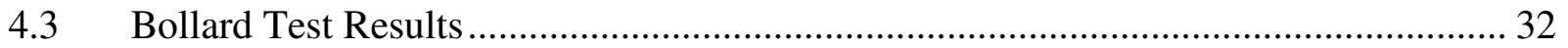

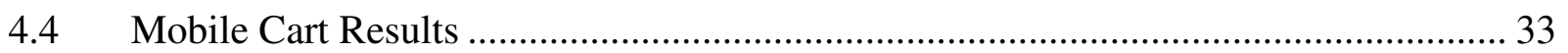

5 Case Study: Designing and Producing a SeaBED Class AUV Propeller ............................... 36

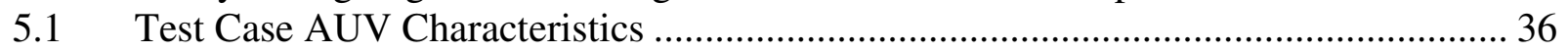

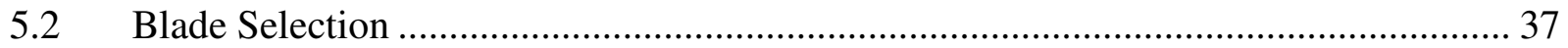

5.3 Propeller Performance Characteristics Comparison ................................................... 39

5.4 Off-the-Shelf Propeller Analysis and Improvement .................................................. 41

5.5 Propeller Hub Assembly and Shaft Design .............................................................. 44

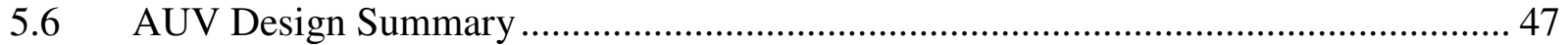

6 Case Study: OpenPVL Propeller Prototype Printable File …………………...................... 50

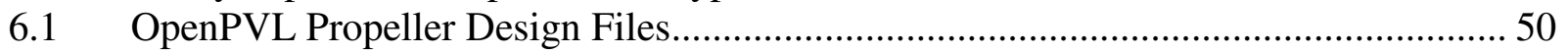

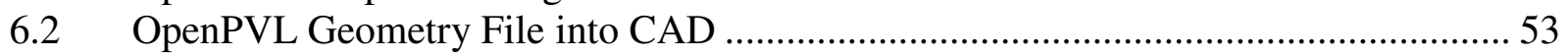

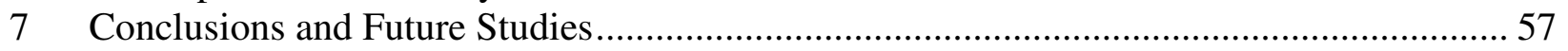

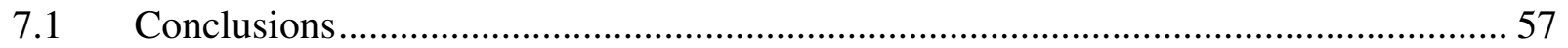

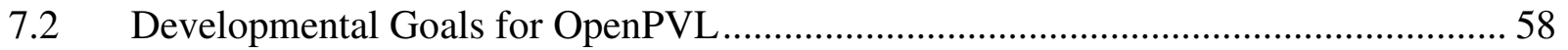

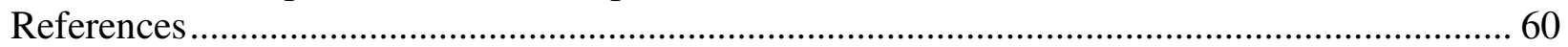

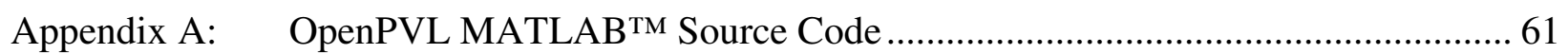

Appendix B: AUV Propeller Design Results ………........................................................... 82

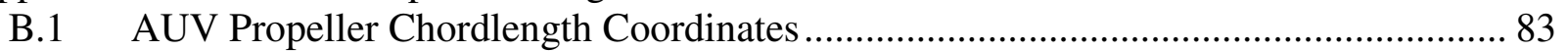

B.2 Geometry used for the US Navy 4148 Propeller Example ............................................ 84

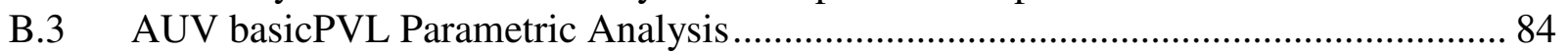

B.4 AUV Off-the-Shelf Propellers Tow-Tank Testing ........................................................ 86

B.5 Actual Measurements for the 3D Printed Propeller Blade............................................. 88

Appendix C: $\quad$ MATLAB ${ }^{\mathrm{TM}}$ Scripting for RHINO ${ }^{\mathrm{TM}}$ : OpenPVL_CADblade.txt..................... 89 


\section{List of Figures}

Figure 2-1: Parametric Blade Design using basicPVL.......................................................... 13

Figure 2-2: Geometry for determining total lifting line velocity, $\mathrm{V}^{*}$, as in [4] .......................... 15

Figure 2-3: Screenshot of the main input screen for OpenPVL code......................................... 16

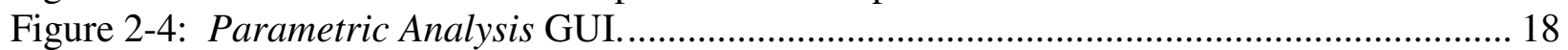

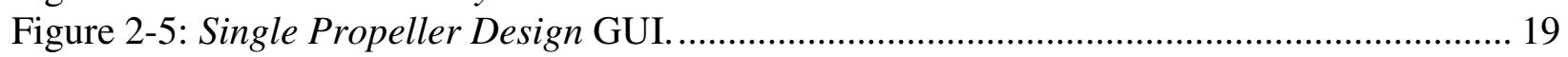

Figure 2-6: Propeller design in CAD (a) and the 3D printed result (b).................................... 20

Figure 3-1: OpenPVL design-to-prototype process............................................................... 22

Figure 3-2: Screenshot of the propeller blade and support structure generated by the StrataSys ${ }^{\mathrm{TM}}$

Insight software. This is the design blade described in more detail in Chapter 5................ 24

Figure 3-3: Time to print estimate for propellers of varying diameters. .................................... 25

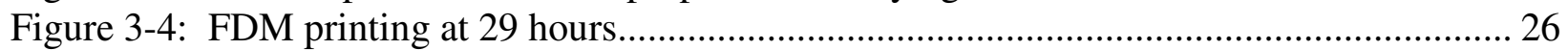

Figure 4-1: Required thrust estimate for a low-speed, high-drag AUV. .................................. 29

Figure 4-2: Tow-tank carriage. ...................................................................................... 30

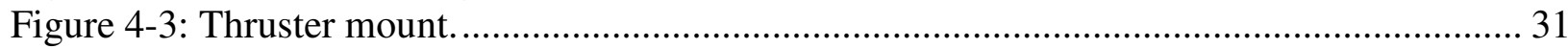

Figure 4-4: Off-the-shelf propeller Bollard Test results.......................................................... 32

Figure 4-5: Bollard Test comparison of thrust, propeller RPM and power input with respect to

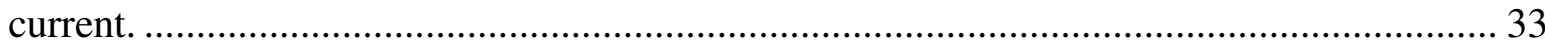

Figure 4-6: Moving Cart Test results. All data ABOVE the dotted line meet the calculated drag requirements for the vehicle..................................................................................... 34

Figure 4-7: Off-the-shelf propeller forward Mobile Cart test results, thrust coefficient, $\mathrm{K}_{\mathrm{T}}$ vs. the

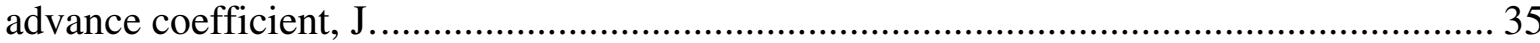

Figure 5-1: Efficiency for a 3-bladed propeller. .................................................................. 38

Figure 5-2: Efficiency and $\mathrm{C}_{\mathrm{L}}$ for varying hub diameters and $\mathrm{z}=3$ blades, $\mathrm{N}=2 \mathrm{RPS}, \mathrm{D}=$ $0.594 \mathrm{~m}$.

Figure 5-3: Efficiency vs. variation of blade hubs for different diameters of the off-the-shelf

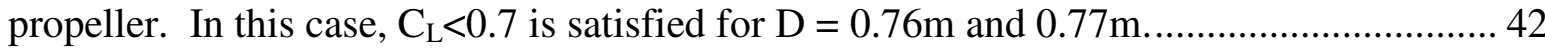

Figure 5-4: MATLAB ${ }^{\mathrm{TM}}$ representation of optimal modified off-the shelf propeller.................. 43

Figure 5-5: MATLAB ${ }^{\mathrm{TM}}$ representation of final propeller design................................................ 44

Figure 5-6: Hub design in CAD (a), and machined (b). ............................................................ 44

Figure 5-7: Modified thruster shaft design in CAD (a), and machined (b). ................................ 45

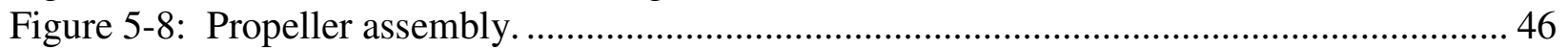

Figure 5-9: Propeller geometry validation, where the actual 3D printed blade is shown in comparison to the desired blade geometry........................................................................... 47

Figure 6-1: Using RHINO ${ }^{\mathrm{TM}}$ to create a .STL file with the 4148-based model propeller............ 55

Figure 6-2: Sample propeller .STL file using OpenPVL 4148 geometry....................................... 55

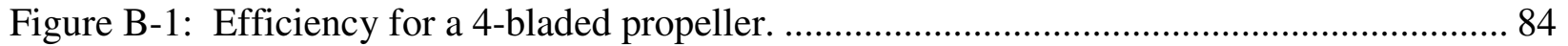

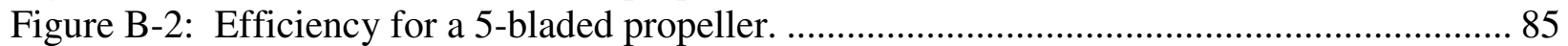

Figure B-3: Efficiency for a 6-bladed propeller. .................................................................... 85 


\section{List of Tables}

Table 3-1: StrataSys ${ }^{\text {TM }}$ Titan FDM Specifications. .......................................................... 23

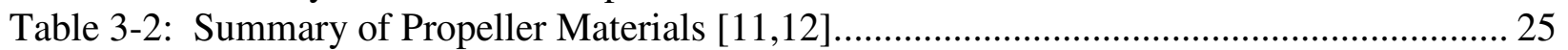

Table 5-1. Final Optimized Propeller Performance vs. Radius Position .................................. 39

Table 5-2: Blade geometry for optimized propeller, using ML type NACA, $a=0.8$, and NACA-

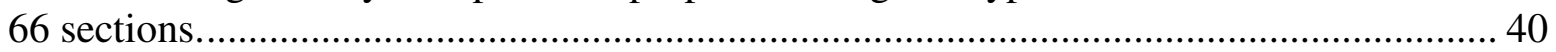

Table 5-3: Off-the-shelf propeller performance vs. radius position. ...................................... 40

Table 5-4: Blade geometry for the off-the-shelf propeller, using ML type NACA, a=0.8, and

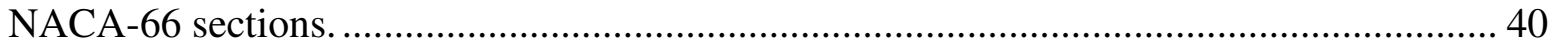

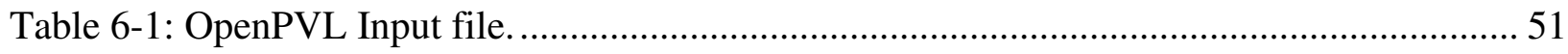

Table 6-2: OpenPVL Output file. .............................................................................. 52

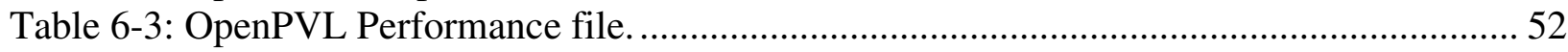

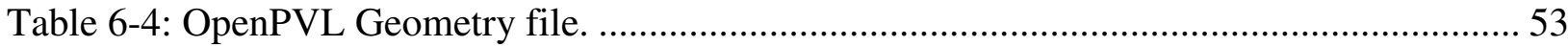

Table B-1: Chordlength distribution of an off-the-shelf propeller. ..................................... 83

Table B-2: Chordlength distribution of an optimized AUV propeller................................... 83

Table B-3: Forward and reverse numerical values for the Bollard Test................................. 86

Table B-4: Forward and reverse numerical values for the moving Cart Test...........................87

Table B-5: Actual Measurements of the 3D Printed Propeller.............................................. 88 


\section{General Nomenclature}

\begin{tabular}{|c|c|c|c|}
\hline $\mathrm{c}$ & section chord length & $\mathrm{t}_{\mathrm{o}} / \mathrm{c}$ & thickness ratio \\
\hline $\mathrm{c} / \mathrm{D}$ & ratio of chordlength to diameter & $\mathrm{u}_{\mathrm{a}}$ & induced axial velocity \\
\hline $\mathrm{C}_{\mathrm{D}}$ & drag coefficient & $\mathrm{u}_{\mathrm{t}}$ & induced tangential velocity \\
\hline $\mathrm{C}_{\mathrm{L}}$ & lift coefficient & $\mathrm{V}_{\mathrm{a}} / \mathrm{V}_{\mathrm{s}}$ & $\begin{array}{l}\text { axial inflow velocity ratio ( is } \\
1.0, \text { assuming uniform } \\
\text { inflow) }\end{array}$ \\
\hline$C_{L C H A R T}$ & $\begin{array}{l}\text { lift coef. From NACA } a=0.8 \text { data } \\
\left(\mathrm{C}_{\mathrm{L}}=1.0\right)\end{array}$ & $\mathrm{V}_{\mathrm{s}}$ & ship (vehicle) velocity \\
\hline$C_{p}$ & propeller power coefficient & $\mathrm{V}_{\mathrm{t}} / \mathrm{V}_{\mathrm{s}}$ & $\begin{array}{l}\text { tangential inflow velocity } \\
\text { ratio ( is } 0.0, \text { assuming } \\
\text { uniform inflow) }\end{array}$ \\
\hline $\mathrm{C}_{\text {Pmin }}$ & $\begin{array}{l}\text { local pressure distribution } \\
\text { coefficient }\end{array}$ & $\mathrm{V}^{*}$ & total inflow velocity \\
\hline $\mathrm{C}_{\mathrm{q}}$ & required torque & $\mathrm{Z}$ & number of blades \\
\hline$C_{T}$ & thrust loading coefficient & & \\
\hline $\mathrm{D}$ & Diameter & & \\
\hline$\underline{f_{o}}$ & camber ratio & $\Delta \alpha$ & Angle of attack variation due \\
\hline$c$ & & & to non-uniform flow \\
\hline$f_{o C H A R T}$ & $\begin{array}{l}\max \text { camber ratio from NACA } \\
\mathrm{a}=0.8 \text { data }\left(f_{o}=0.679\right)\end{array}$ & $\beta$ & $\begin{array}{l}\text { undisturbed flow angle } \\
\text { (relative to blade) }\end{array}$ \\
\hline G & $\begin{array}{l}\text { non-dimensional circulation, } \\
G=\frac{\Gamma}{\pi R V_{s}}\end{array}$ & $\beta_{i}$ & hydrodynamic pitch angle \\
\hline $\mathrm{H}$ & underwater vehicle operation depth & $\Gamma$ & vortex circulation strength \\
\hline$J$ & advance coefficient, $J=V_{s} / N D$ & $\eta$ & Efficiency \\
\hline$K_{q}$ & torque coefficient & $\rho$ & Water density \\
\hline$K_{T}$ & propeller thrust & $\sigma$ & cavitation number, \\
\hline & $K_{T}=C_{T} J^{2} \pi / 8$ & & $\sigma=\frac{P a t m+\rho g h-P v a p}{\frac{1}{2} \rho V^{*}}$ \\
\hline
\end{tabular}

N propeller Revolutions Per Second

$\mathrm{P}_{\mathrm{atm}} \quad$ atmospheric pressure

$\mathrm{P}_{\text {vap }} \quad$ vapor pressure

P/D ratio of pitch over diameter

$r / R \quad$ ratio of the radial location, $r$, to the total length of the blade radius, $R$ 


\section{Introduction and Background}

\subsection{Motivation}

Autonomous Underwater Vehicles (AUVs) serve in scientific research and in military operations. Engineers need an adaptable tool that can streamline the propeller design process for a wide range of vehicles, including AUVs. REMUS AUVs have experienced success through use of their own propeller designs, yet such design capabilities need to be made available in the form of open source code, such as OpenProp [1]. In some cases, off-the-shelf propellers are used for underwater vehicles. Off-the-shelf designs are easier to obtain than a customdesigned propeller, yet they are not optimized for the capabilities of a specific vehicle. It is important to consider that a wide variety of underwater vehicles exist. Unique vehicle characteristics call for a tool that has the capacity to design propellers for a specific application.

Underwater propellers are sometimes not optimized due to factors of cost and availability. In designing a vehicle, off-the-shelf components can be very desirable. Traditionally, it would be much more costly, difficult, and time-consuming to design and fabricate a propeller than it would be to order one that has already been tested and can easily be replaced. Some AUV designs, including the SeaBED and ABE varieties of AUVs developed in the Deep Submergence Lab at the Woods Hole Oceanographic Institute (WHOI), incorporate a commercially available model airplane propeller. Manufactured propellers can be appropriately analyzed and chosen by inputting various off-the-shelf blade geometries into OpenPVL and then evaluating the commercial options to determine the propeller that is best fit to serve a vehicle in operation. In this way, off-the-shelf propeller users can benefit from the streamlined design-toprototype process OpenPVL offers users.

In a situation in which fabricating and testing propellers is prohibitively expensive, or it is desirable to use existing propellers, modifications can be made to improve performance in propeller efficiency. This analysis seeks to provide a means of designing and manufacturing propellers for specific AUV operations, while contributing to methods for improving performance using off-the-shelf model airplane propellers. 
Use of propellers in aircraft and ships, both military and commercial, commands the development of modern propeller design tools [2]. Today, computers allow a detailed optimization of propellers. Propeller vortex lattice methods developed by J. Kerwin represent the propeller as a vortex lifting line. Efficiency is maximized by computing the optimal radial distribution of loading (circulation). Hub effects are represented with image vortices and the wake is represented as constant diameter helical vortices aligned with the hydrodynamic pitch of the propeller flow. After optimizing an initial design, the propeller performance undergoes two-dimensional analysis, including determining minimum pressure coefficients and local lift coefficients. Rigorous design methods use multiple iterations of many parameters to calculate a highly efficient propeller design [3].

\subsection{Recent Propeller Design Efforts}

Vortex lifting line codes provide a powerful method for assessing the characteristics of a propeller. In the initial analysis, Propeller Vortex Lifting Line (PVL) code, was used in combination with MATLAB ${ }^{T M}$ m-files [4]. The m-file created to use with PVL, basicPVL.m, was a developmental code used prior to to the May 2007 release of Hsin-Lung Chung's open source MIT Propeller Vortex Lifting Line code, (MPVL) [5]. MPVL can be utilized to perform both a parametric propeller analysis, and a single propeller design.

The most recently developed design tool is OpenPVL, which is an open source propeller design tool that has the capability to manufacture propeller blades from desired design inputs. The OpenProp suite (including OpenPVL) is based on the FORTRAN programs originally developed by Professor J. Kerwin at MIT in 2001 [4], translated into MATLAB ${ }^{\text {TM }}$ as an open source MPVL code released by Hsin-Lung Chung in May 2007 [5]. OpenPVL operates using an evolved MPVL code, while expanding upon MPVL's applications, and it has been modified to create scripting for $3 D$ printable files through the CAD interface commercial software RHINOTM. OpenPVL creates a user-friendly interface to quickly input design characteristics and output performance data and propeller blade geometry. Source code for OpenPVL.m can be found in Appendix A. OpenPVL files, including the most recent updates, can be obtained along with the OpenProp suite at http://web.mit.edu/openprop/. 


\section{Lifting Line Propeller Design Codes}

\subsection{An Introduction to Design Techniques}

There are many methods for propeller design [4,6], but in this study, propeller vortex lifting line code (both basicPVL.m and OpenPVL.m) were analyzed using cavitation considerations to meet the AUV specifications [3]. These methods lead to designing an optimal propeller, as well as calculating the efficiency of existing propellers, which determined performance flaws. The resulting analysis produced recommendations for altering the geometry of off-the-shelf propellers to improve overall efficiency, while considering the effects of minimum pressure coefficients and ranges of lift coefficient. Aspects of interest in propeller design included the following: the vehicle's desired thrust, cruise speed, number of propellers, propeller location, and depth of vehicle. The design additionally considered the affects of blade number, rate of propeller rotation, chord length distribution, propeller diameter, and hub size.

This study made several assumptions. First, the propellers were located far enough away from the hulls of the AUV, which left axial velocity, $V_{s}$, unaffected. The ratio of axial velocity to design speed was such that $V_{a} / V_{s}=1.0$. Hull wake and boundary layer effects were not considered in the cases studies presented. However, these effects can be accounted for by using basicPVL, which handles radial variation both axial and tangential velocities. Additionally, hull diameter was insignificant due to the location of the thrusters away from the body of the AUV; thruster diameter was small relative to the propeller diameter. Also, the propeller design code did not automatically check for cavitation effects, but was analyzed by hand using Brockett diagrams. Furthermore, the required thrust was determined for the design speed, and calculated per propeller, not as the total required thrust for the vehicle.

The code basicPVL was designed as an intermediate code to perform analysis during the time that the GUI for OpenPVL was being developed. The analysis considered both the optimal propeller design for the given design criteria, as well as the efficiency of the existing propeller. Efficiency $(\eta)$, is determined by the ratio of required thrust times the wake to the power coefficient. The actuator disk efficiency can be calculated in addition to $\eta$. If a value 
higher than the actuator disk efficiency is found, it can be eliminated as erroneous. The actuator disk efficiency is calculated as in Equation 2.1 [4]:

$$
\eta_{\text {ActuatorDisk }}=2 /\left(1+\left(1+K_{T}\right)^{1 / 2}\right)
$$

This calculation serves as an added check to determine if the calculated efficiency $(\eta)$ is correct. In finding an initial propeller design, PVL.exe was used with input variables, as in [4], including the advance coefficient, $\mathrm{J}$, the desired thrust coefficient, $C_{T}$, and the axial flow ratio, $\mathrm{V}_{\mathrm{a}} / \mathrm{V}_{\mathrm{s}}$.

A function was created to determine the effects of axial flow for alternative propeller placement or diameter of a body affecting fluid flow entering the propeller [7]. ${ }^{1}$ The axial flow modeling considers several important factors. The first factor is in the case that the ratio of the propeller blade diameter to the hull diameter is not equal to one. The data was extended to include a case of a ratio of up to 1.8. Additionally, the practical assumption was made that axial flow is constant for all $r / R$ values less than 0.225 . Otherwise, the spline would model a value of zero or less at the hub, which is not possible.

Other variables, such as the number of vortex panels over the radius, iterations in the wake alignment, hub image, hub vortex radius/hub radius, number of input radii, hub unloading factor, tip unloading factor, swirl cancellation factor, radial position ( $r / R)$, chord to diameter ratio (c/D), and blade section drag $\left(C_{D}\right)$, were kept at acceptable values from example input files to the PVL code. The $c / D$ values were scaled automatically with respect to $r / R$ values using a spline function.

\subsection{Blade Design Using basicPVL}

Blade selection was determined by parameterization of a range of acceptable vehicle design speeds, propeller diameters, and number of blades. A range of values were chosen for the blade number $(\mathrm{z})$, revolutions per second $(\mathrm{N})$, and the diameter of the propeller $(\mathrm{D})$. Values for $\mathrm{z}, \mathrm{N}$ and $\mathrm{D}$ were plotted versus propeller efficiency, and shown as contour lines on a 3D plot.

\footnotetext{
${ }^{1}$ Data from the experimental Huang Body 1 relating axial flow to radial position was splined such that it returns values for axial flow for any range and any spacing of values for radial position.
} 
The code for basicPVL presents an understanding of principles of propeller design. Chordlength optimization were crudely accomplished by a manual process. The input values were somewhat limited and desired values for the parameters were hand-typed for each case tested. This method of parameterization lead to the choice of an initial propeller design, as shown in Fig. 2-1.

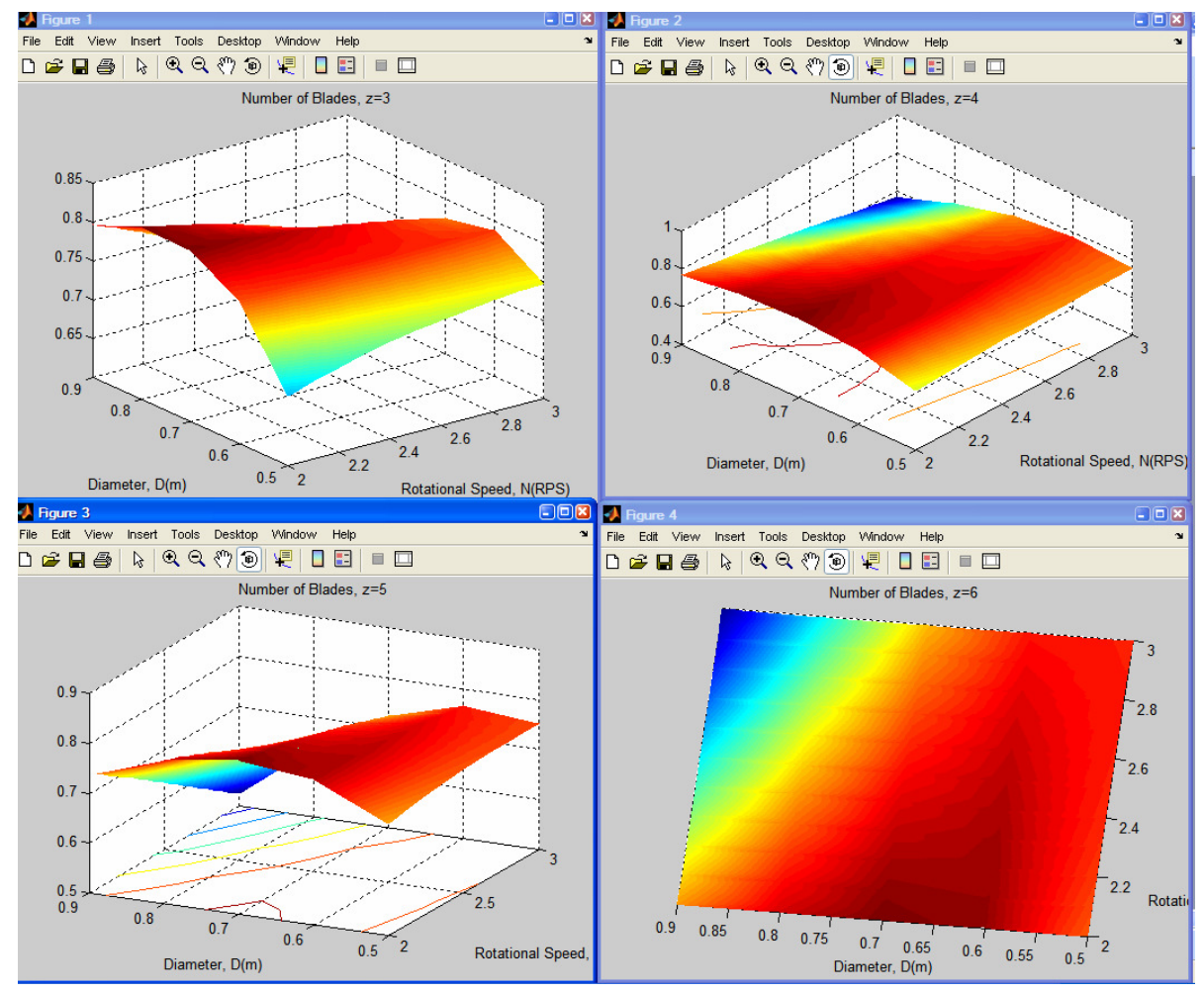

Figure 2-1: Parametric Blade Design using basicPVL.

The intial blade shape parameters, such as chord, section thickness, rake and skew radial distributions are input by the designer. After the intitial run of the code, the chord distribution can be adjusted manually to adjust the section lift and cavitation performance of the blade. The blade section drag coefficient distribution is also user input to account for blade viscous drag effects. For typical NACA foils operating with lift coefficients between 0-0.5 and an assumed uniform blade section drag, $C_{D}=0.008$ is used as an estimate in practical applications. Typically, the designer uses a distribution of lift coefficients which is higher at the hub, and tapers to lower at the tip for added strength and stall prevention. Once the shape of the propeller is chosen, the design process continues with an optimization of chordlength. Other 
slight parameter adjustments improve overall efficiency. It is in the section solver that pressure distribution and the lift coefficient are considered. The finely adjusted ratio of chordlength over diameter $(\mathrm{c} / \mathrm{D})$ depends on an appropriate lift coefficient, $\mathrm{C}_{\llcorner}$. The calculated drag coefficient, $C_{D}$, remains relatively constant for lift coefficients from $0.2-0.5$, with a higher lift coefficient at the hub, and a lower lift coefficient at the tip of the blade [8]. The lift coefficient at the tip must be lower than at the hub due to blade strength limitations [3]. By remaining within this range of lift coefficients, lift is maximized with minimal sacrifice of efficiency to drag. The lift coefficient can be determined by dimensionalizing gamma, $\mathrm{G}$, at each radial location, using the following two equations (2.1-2.2)[3]:

First, $\quad \Gamma=2 \pi G V_{s} R$

Then, $C_{L}=\frac{2 \Gamma}{V^{*} c}$

The total velocity of the lifting line, $\mathrm{V}^{*}$, can be found by using $\beta_{i}$, and calculating $\omega r$ less ut $^{\star}$ given from APLOT.plt in PVL. Figure 2.2 shows the representation of the velocity vectors at a particular radius on the lifting line blade. The onflow to the section is $V$ and the induced velocity components $u^{*}$ and $u^{*}{ }^{*}$ represent the contributions of the propeller, hub, and wake vortex structures to the overall flow. The total flow at the lifting line, including the propeller induction is $\mathrm{V}^{*}$, and the hydrodynamic pitch angle $\beta_{i}$ represents the flow angle seen by the blade section used for setting the section camber and angle of attack. The 2D sections are then set at the ideal angle of attack for the chosen section type and the camber is scaled to achieve the computed lift coefficient for the section. In this way, the blade geometry at each radii is determined, resulting in a complete blade geometry. 


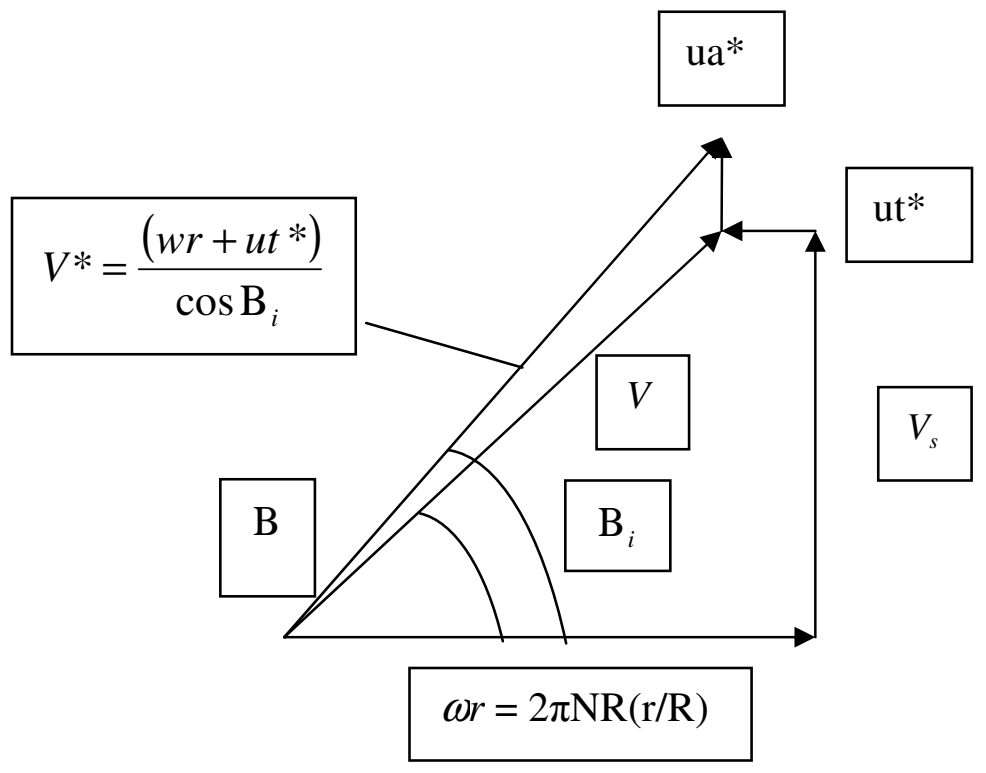

Figure 2-2: Geometry for determining total lifting line velocity, $V^{*}$, as in [4].

Cavitation is an important design consideration for propellers operating at shallow depth or at high loading. Preliminary cavitation prediction and design is achieved using the procedure of Brockett [9], using the Brocket diagram method for a cavitation-free blade design. At each blade radii, the minimum local operating depths of the section is computed using equation 2.3 and the section cavitation number is computed using equation 2.4. The axial inflow variation is estimated by the designer and converted into an angle of attack variation by applying the inflow variation to the velocity triangle (Fig. 2-2) and calculating the change in inflow angle ( $\beta$ ). Next, the Brocket diagrams are used to select the section thickness in order for the foil to achieve cavitation-free performance. The section chordlength can be increased until the desired performance is achieved. In addition to being less efficient, partial cavitation creates bubbles which can damage propeller blades through pitting [10]. The cavitation number can be compared to the pressure coefficient such that cavitation will not occur if $\sigma>\mathrm{C}_{\mathrm{Pmin}}$. The value for the cavitation number, $\sigma$, can be found from the following equations (2.3-2.4) [4]:

$$
\begin{aligned}
& h=h_{o}-(r / R)\left(\frac{D}{2}\right), \text { where } h_{o}=\text { operating depth. } \\
& \sigma=\frac{\text { Patm }+\rho g h-P \text { vap }}{\frac{1}{2} \rho V^{*}}
\end{aligned}
$$


The Brockett diagram utilizes the NACA-66 sections [9] in finding the camber ratio, $\mathrm{f}_{\mathrm{o}} / \mathrm{c}$, and the inflow variation bucket width, $\Delta \alpha$, is used to determine the thickness ratio, $t_{0} / c$, and local pressure distribution coefficient, $\mathrm{C}_{\mathrm{Pmin}_{\mathrm{in}}}[4]$.

\subsection{Design Techniques Using OpenPVL}

OpenPVL is a propeller design tool that is based on the same vortex lattice lifting line theory as basicPVL. However, OpenPVL offers a variety of advanced features, including:

- A user-friendly MATLABTM GUI

- Ability to save valuable input and output text files for each propeller design

- Capacity to write a script file for 3D printing from the design geometry output

Figure 2-3 shows the main input screen for the OpenPVL code. Two options are available at this level, Parametric Analysis and Single Propeller Design.

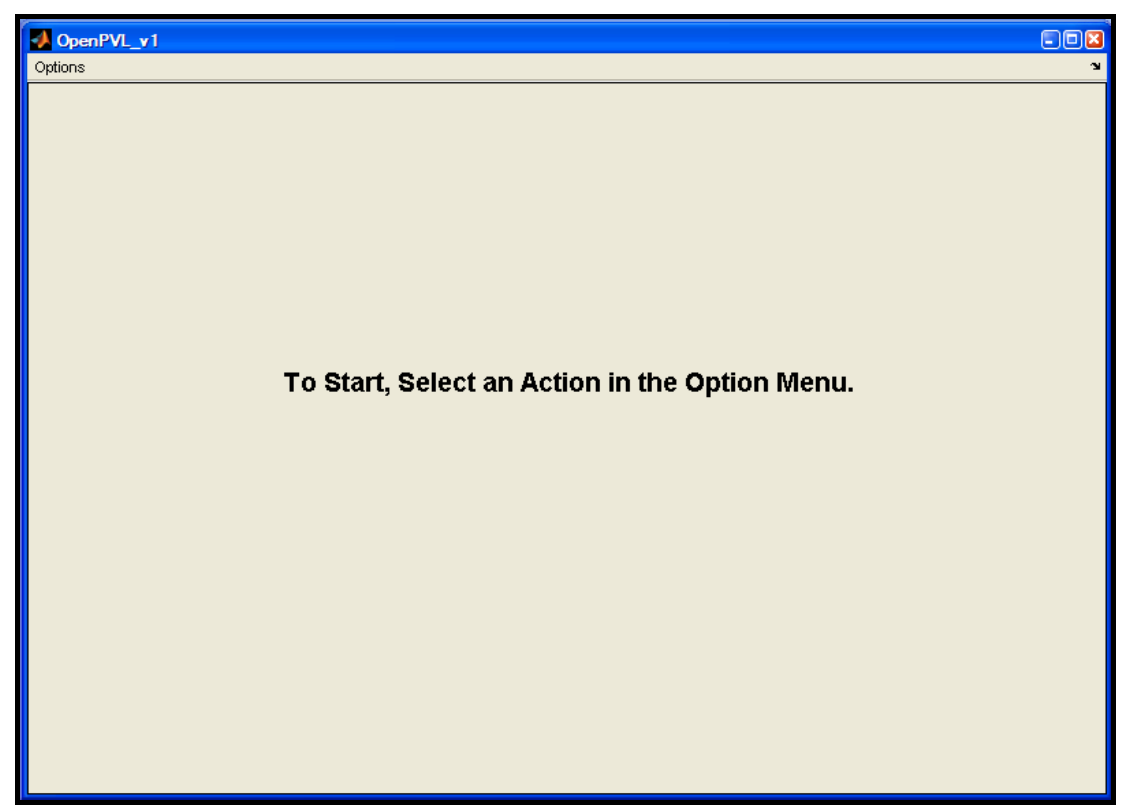

Figure 2-3: Screenshot of the main input screen for OpenPVL code. 


\subsubsection{Parametric Analysis}

Three parameters provide the foundation for propeller design: the number of blades, the propeller speed, and the propeller diameter. Various combinations of these three key parameters result in different efficiencies. Thus, a parametric study allows for propeller parameter optimization. The Parametric Analysis GUI is a computational tool that calculates and graphically represents propeller efficiency. Figure 2-4 shows the parametric analysis GUI, which includes the user input fields required to run the analysis. OpenPVL is tailored to a propeller user's design needs, therefore, the Parametric Analysis GUI requires user input for the following characteristics:

- Number of blades

- Propeller speed

- Propeller diameter

- Required thrust

- Ship speed

- Hub diameter

- Number of vortex panels over the radius

- Maximum number of iterations in wake alignment

- Ratio of hub vortex radius to hub radius

- Number of input radii

- Hub and tip unloading factor

- Swirl cancellation factor

- Water density

- Hub image flag

All of the fields within the GUI are populated with initial values, based on the US Navy 4148 propeller, as a guide to users. Each of the input fields are modifiable and Parametric Analysis can run any desired number of times without having to exit the program [5]. 


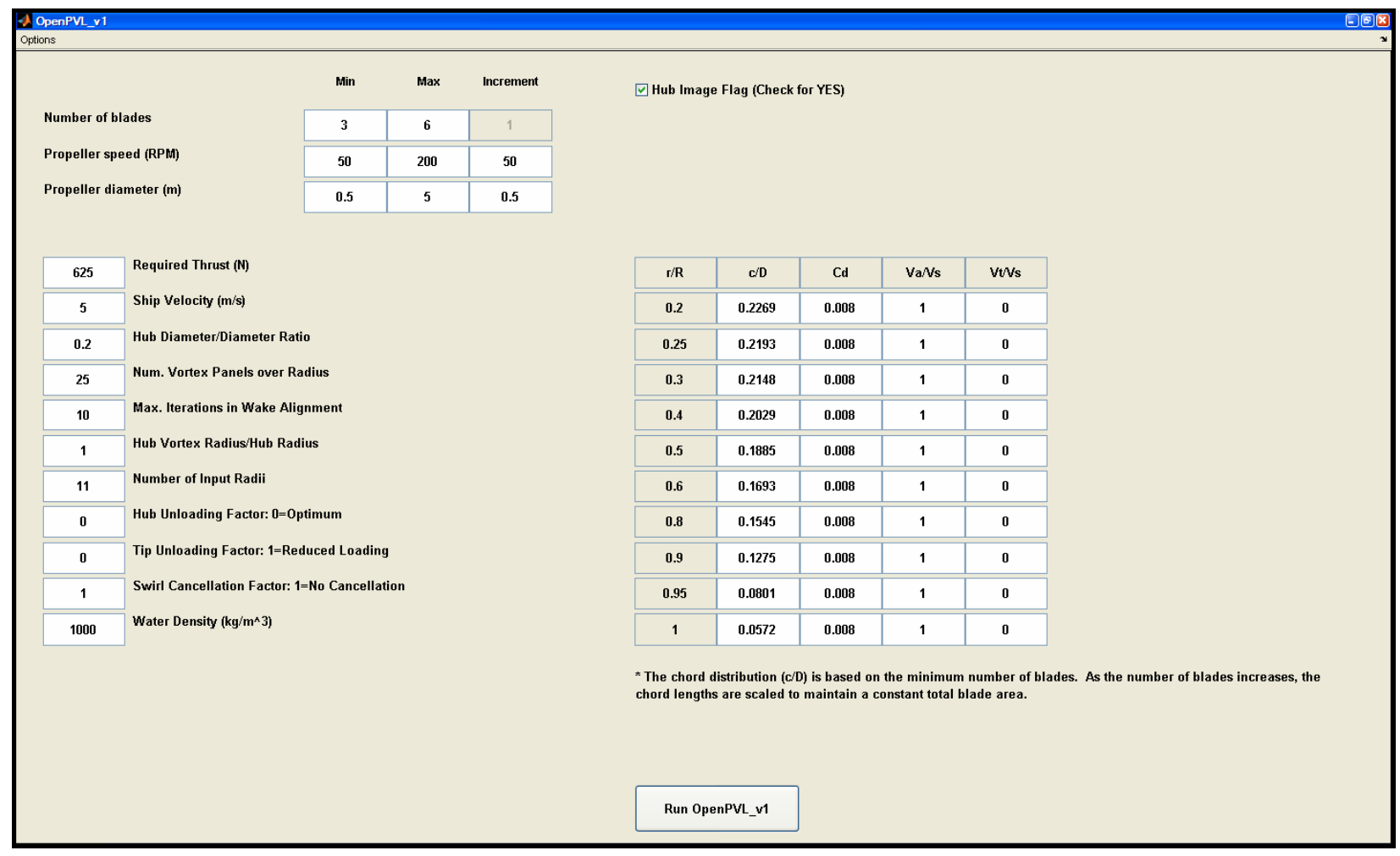

Figure 2-4: Parametric Analysis GUI.

\subsubsection{Single Propeller Design}

Once the parameters for a propeller with a viable efficiency curve has been established, the desired inputs are entered into the Single Propeller Design GUI of OpenPVL. Figure 2-5 shows the single propeller design GUI. Determining the geometry for a single propeller utilizes both the results from the propeller parameterization, as well as additional inputs, resulting in a user-specific design. Input fields entered for the Parametric Analysis are populated with the same values for the Single Propeller Design. There are also several additional input fields, including: shaft centerline depth, inflow variation, ideal angle of attack, and the number of points over the chord. Additionally, two types of meanlines are available within the program: the NACA $a=0.8$ and the parabolic meanline. The thickness forms available include: NACA 65 A010, elliptical, and parabolic. OpenPVL is easily modified to accommodate additional meanlines and thickness forms. 


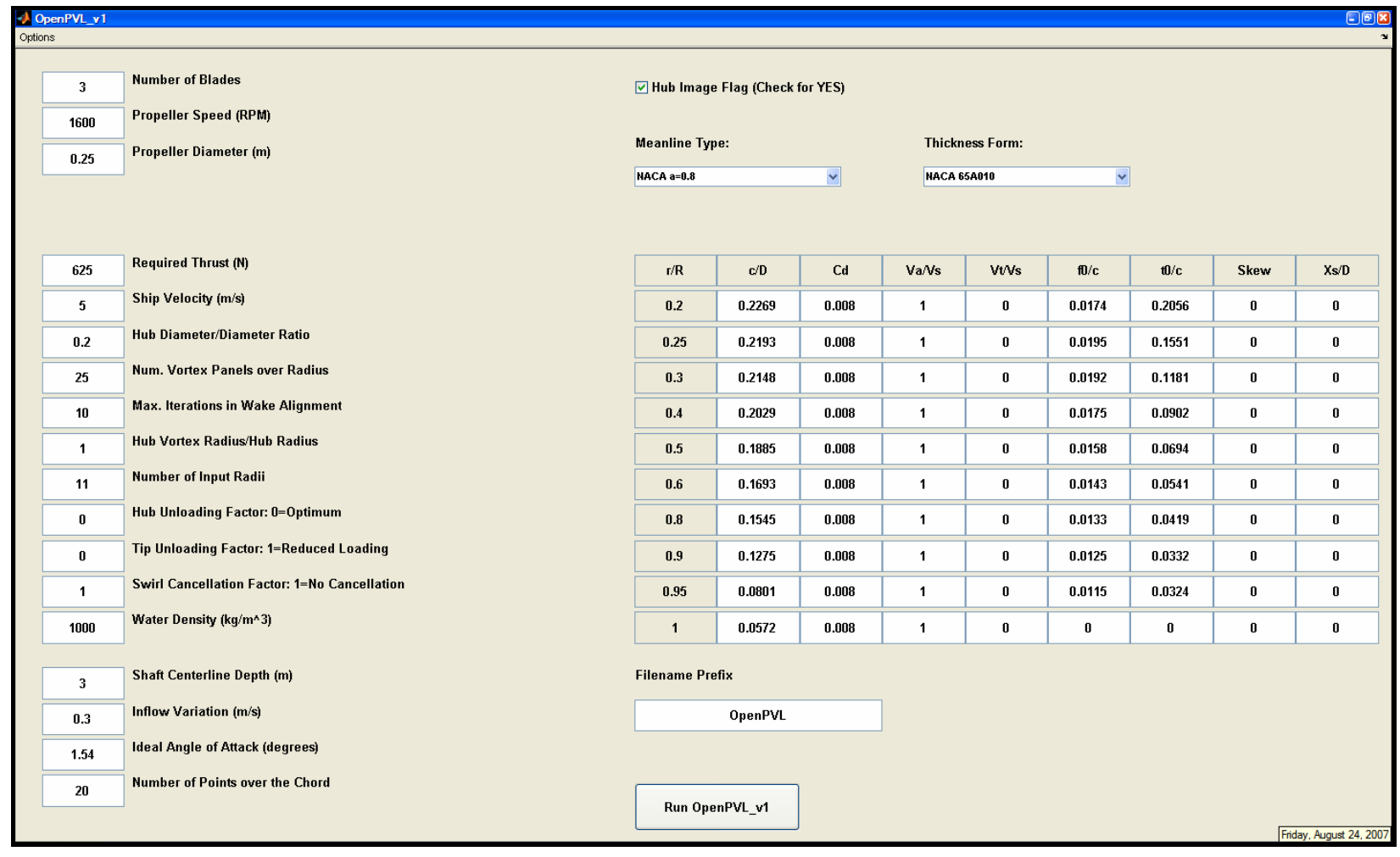

Figure 2-5: Single Propeller Design GUI.

A single propeller can be designed and quickly evaluated graphically. One of the file outputs of the Single Propeller Design is the blade geometry. This feature of OpenPVL automatically transforms $\mathrm{x}, \mathrm{y}$ and $\mathrm{z}$ coordinates of the designed propeller blade geometry into a command file that can be read by a CAD program. The user opens the command file in the CAD program RHINOTM, and has the option of saving their design as a useful stereolithography (.STL) file, or as an Initial Graphics Exchange Specification (.IGES) file. Although the command file includes scripting specific to RHINOTM, the propeller geometry can be exported to another CAD program after it is saved in RHINOTM as a file compatible to the other design software. The single propeller design option creates both propeller geometry and a corresponding scripting code which allows the propeller to be created as a .STL file and then printed on a 3D printer, as shown in Fig. 2-6. Propeller designs can be modified and saved with the OpenPVL tools repeatedly, at no cost to the user. 


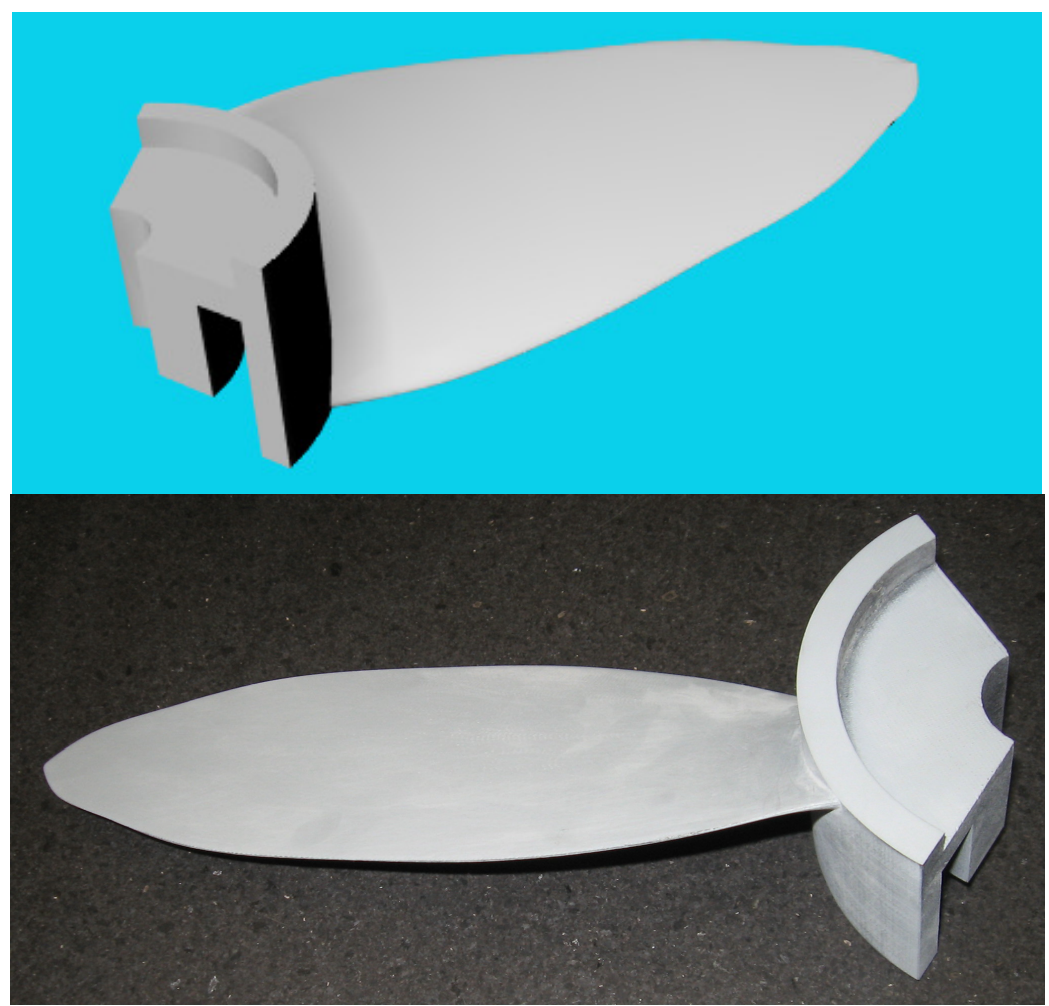

Figure 2-6: Propeller design in CAD (a) and the 3D printed result (b). 


\section{Propeller Printing: from Design Geometry to Prototype}

\subsection{Blade Geometry from OpenPVL to .STL Files}

OpenPVL optimized the propeller design geometry, and then automated the creation of a RHINOTM command file. Using the RHINOTM NURBS modeling program, a blade with the desired geometry was drawn, converted into a .STL file, and was ready to send to a threedimensional printer, as detailed in Chapter 5. OpenPVL uses several simple steps to guide propeller users to rapidly create optimal propeller geometry that can be printed, tested, and ultimately put to use.

OpenPVL allows users with limited backgrounds in propeller design to produce a prototype propeller. In this study, OpenPVL created a file readable by a 3D printer, as illustrated in Fig. 3-1. First, propeller characteristic boundaries were designed with the Parametric Analysis tool in OpenPVL. Desired characteristics of the vehicle were entered into the Parametric Analysis GUI of OpenPVL in order to determine the range of appropriate propeller characteristics that should be used as input for the Single Propeller Design of OpenPVL.

Next, desired design characteristics were entered in the Single Propeller Design option of OpenPVL. The user has the option to create a filename and save a variety of propeller initial inputs and outputs, including geometries that can be read as command files into the RHINOTM CAD program. RHINOTM has the capability to convert the geometry created by the command file into saved .STL and .IGES files, among others. The .STL files can be read and printed into model blades, from which molds may be made. The .IGES files are recognized by a variety of CAD programs. 

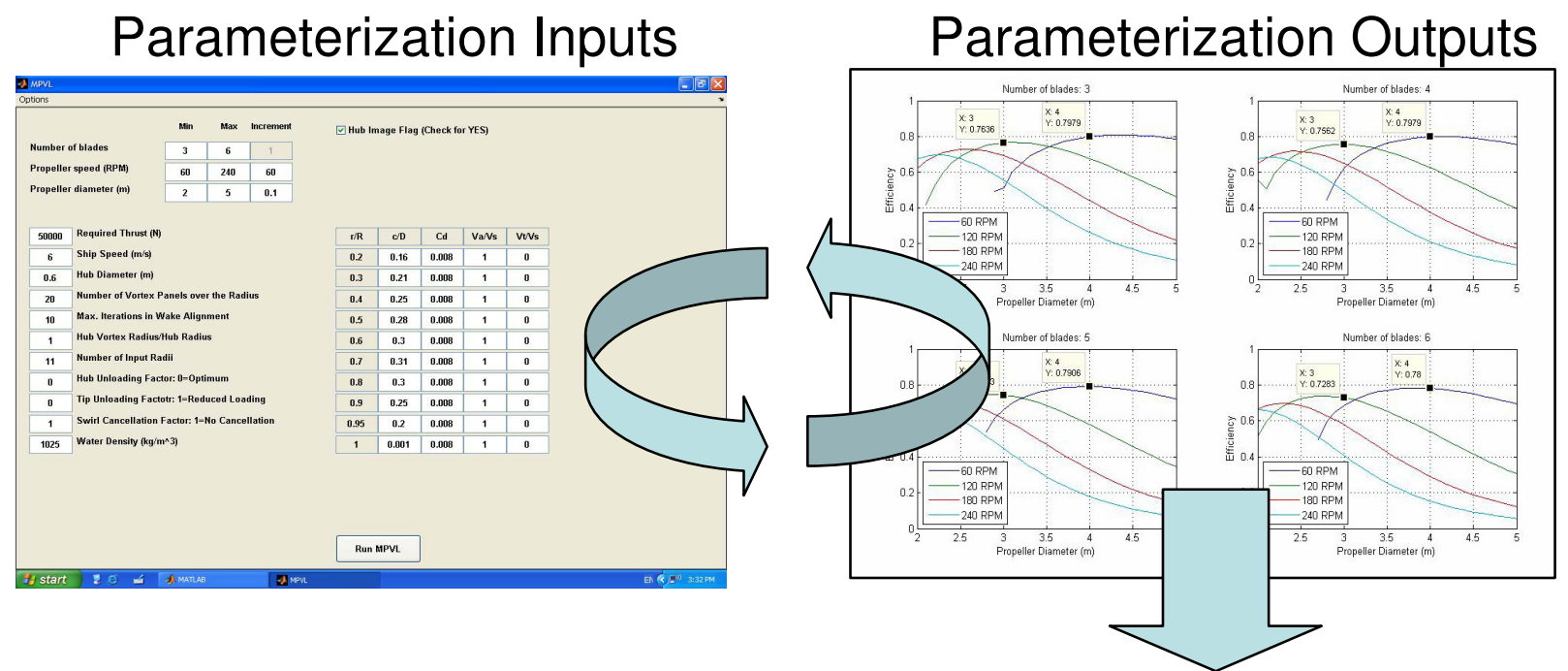

Single Propeller Design Outputs

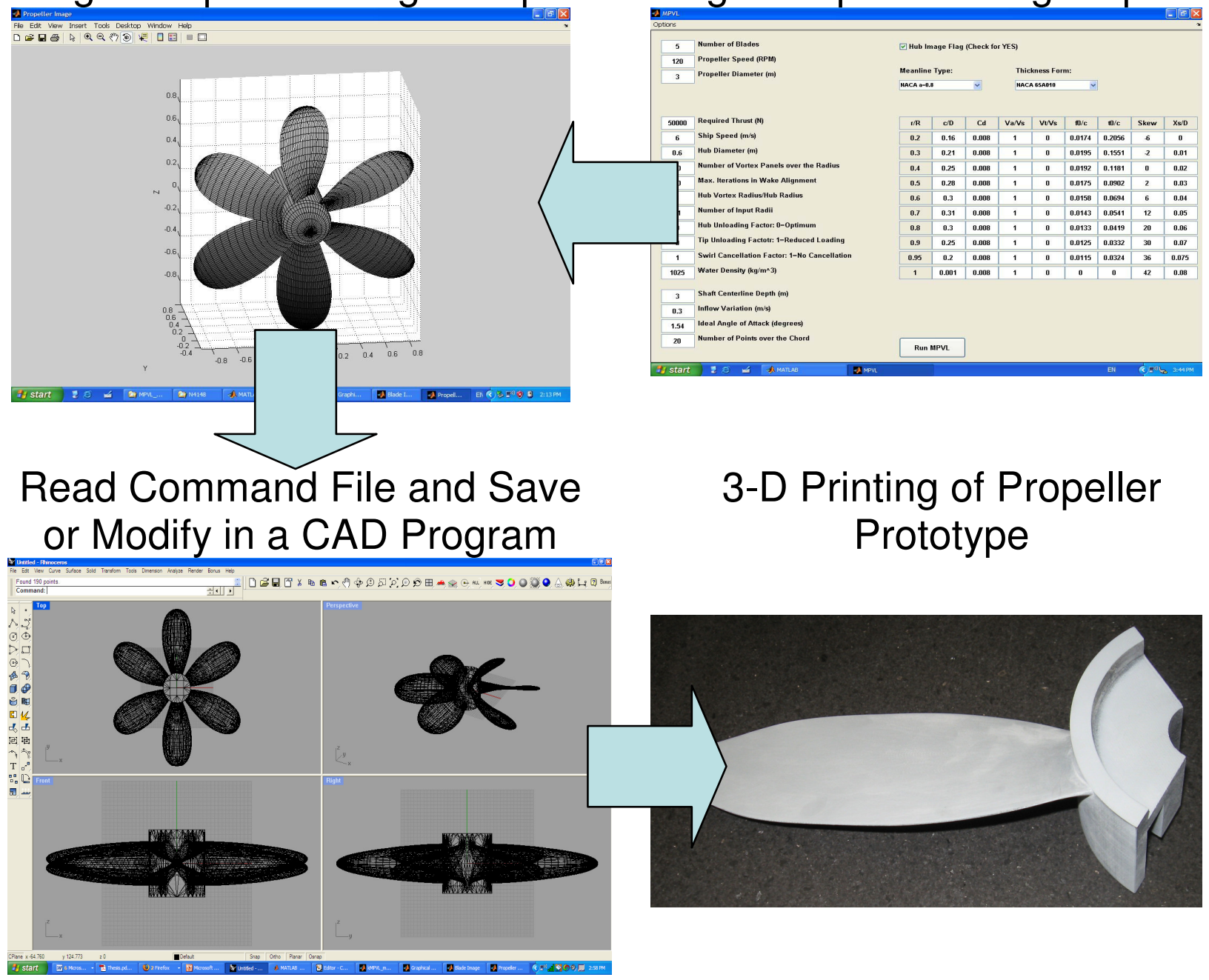

Figure 3-1: OpenPVL design-to-prototype process. 


\subsection{Fusion Deposition Modeling (FDM) Printer Overview}

There are several different types of machines and manufacturers of 3D printing machines, such as FDMs (StrataSys ${ }^{\text {TM TM }}$ and Solidscape ${ }^{\text {TM }}$ ), stereolithography machines (3D Systems ${ }^{\mathrm{TM}}$ ), and printers capable of making 3D design parts or can print a mold (Z-Corp ${ }^{\mathrm{TM}}$ ). All of these machines accept the .STL file format as input. The method of 3D printing available for this study was with an FDM. ${ }^{2}$ FDM printing requires the input of a .STL (Stereolithography) file. Specifications of the 3D printer used in this study are listed in Table 3-1 [11].

Table 3-1: StrataSys ${ }^{\mathrm{TM}}$ Titan FDM Specifications.

Build Volume Within 16 inches by 14 by 16 inches $(\mathrm{LxWxH})$.

Materials acrylonitrile butadiene styrene (ABS), polycarbonate (PC), and PC-ABS

Layer thickness

Ranges from 0.007in to 0.013in

Support structures

$A B S$ and $P C-A B S$ are water-soluble

Material Canisters

Two 92in for the build materials and the support materials

Labor Machine may be unattended during builds, but requires extra labor when changing material type used in the machine

Software .STL files are imported using Insight ${ }^{\mathrm{TM}}$

Once a .STL is input into the FDM printer software, a support structure is automatically created for the input file, as shown in Fig. 3-2. The support structures of ABS and ABS/PC both are built such that they dissolve after printing, during the finishing process. The support structure of polycarbonate must be filed down manually, which leaves room for deviation from the intended geometry, as well as more time invested in the manufacturing process.

\footnotetext{
${ }^{2}$ The specific FDM used in this study was a StrataSys ${ }^{\mathrm{TM}}$ for the Titan FDM.
} 


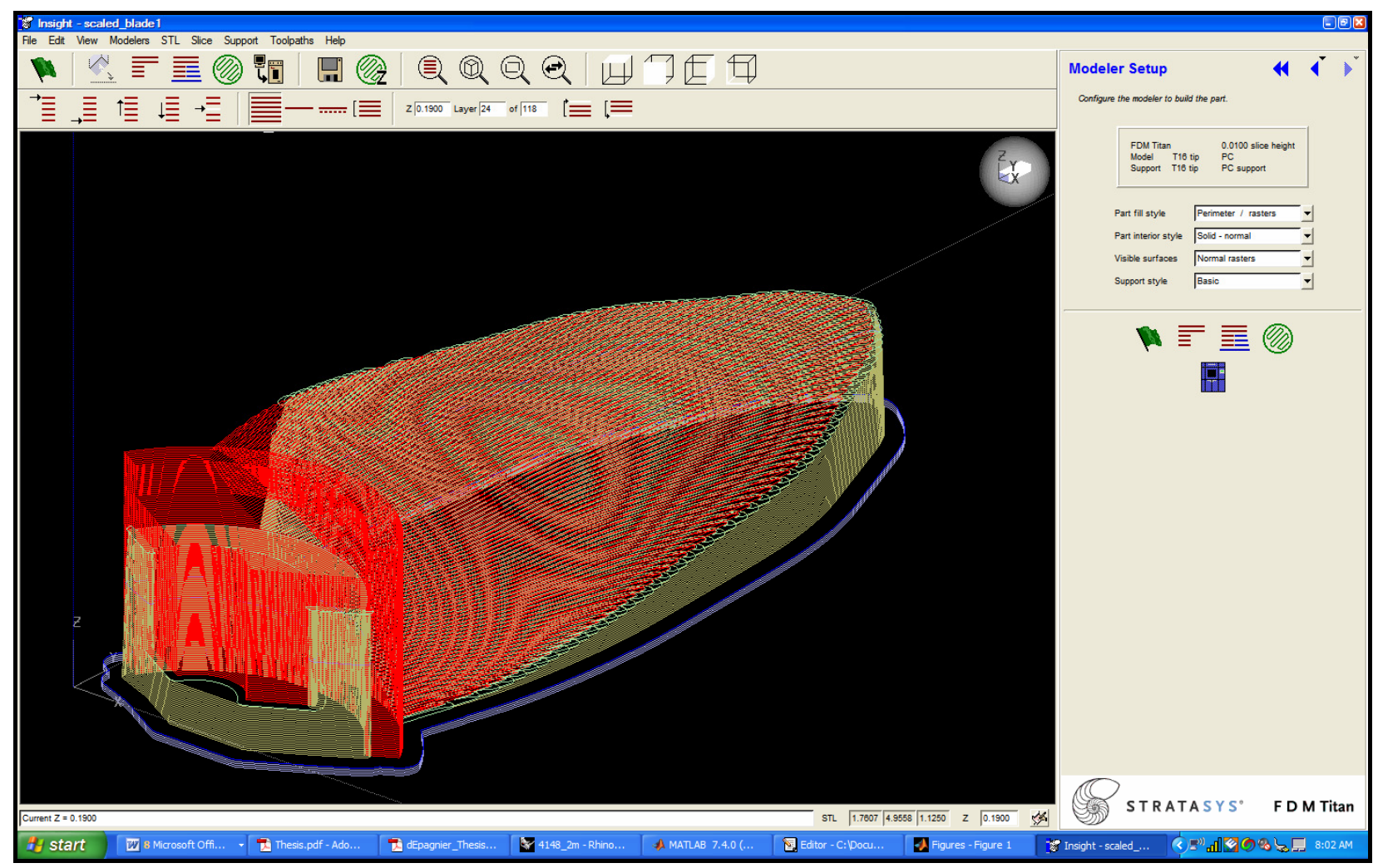

Figure 3-2: Screenshot of the propeller blade and support structure generated by the StrataSys ${ }^{\mathrm{TM}}$ Insight software. This is the design blade described in more detail in Chapter 5.

The FDM printable file generates both a total volume calculation, as well as a printing completion time estimate. The total volume calculation is used by some FDM printer operators to determine the price per volume of the object printed. The time to print the volume of both the propeller and its support structure was modeled for various diameters, as in Fig. 3-3. The plot indicates that the time needed to print a $25 \mathrm{~cm}$ three-bladed propeller prototype takes approximately 44 hours. 


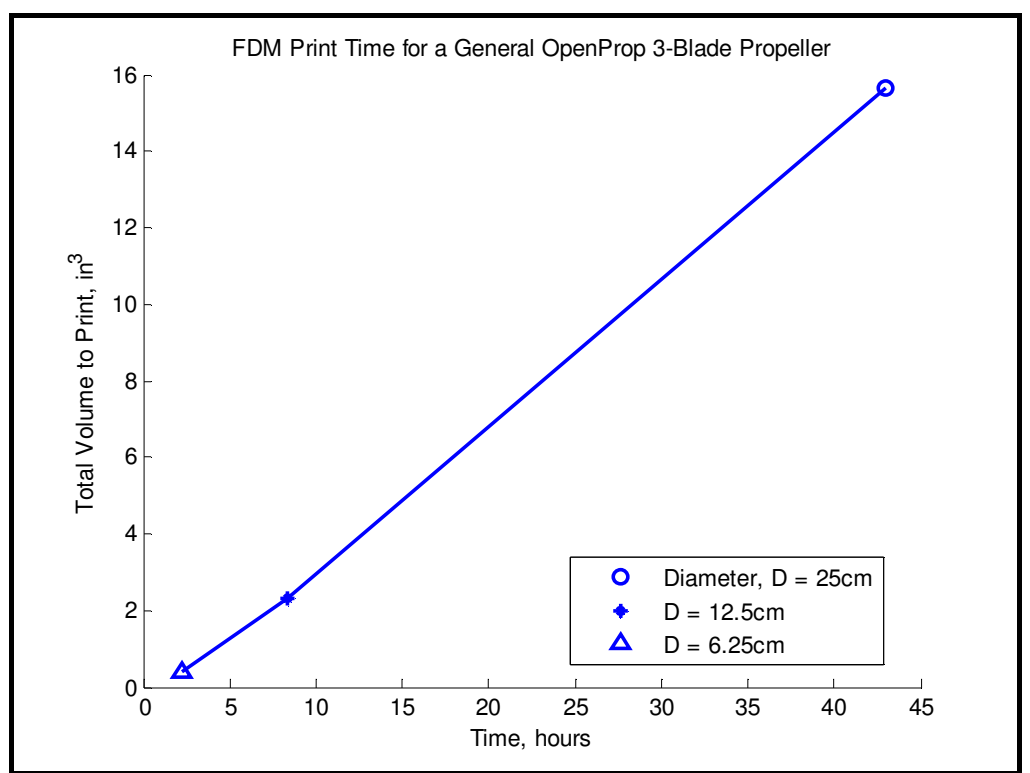

Figure 3-3: Time to print estimate for propellers of varying diameters.

\subsection{Analysis of Materials for Fusion Deposition Modeling}

Material options for a Fusion Deposition Modeling (FDM) printer include three types of plastics: acrylonitrile butadiene styrene (ABS), polycarbonate (PC), and an ABS/PC blend. While ABS solids can be easily filed down to a smooth finish, if necessary, after printing, the material's tensile and flexural strengths and modulus of elasticity are not comparable to that of the ABS/PC material. Additionally, the polycarbonate and the ABS/PC are more rigid than ABS, and therefore undergo less deformation from the desired propeller prototype geometry. A summary of material options for this particular Titan FDM ${ }^{\text {TM }}$ is in Table 3-2.

Table 3-2: Summary of Propeller Materials [11,12].

\begin{tabular}{|c|c|c|c|c|c|}
\hline Material & $\begin{array}{l}\% \text { Water } \\
\text { Absorption } \\
(24 \mathrm{hrs})\end{array}$ & $\begin{array}{l}\text { Hardness } \\
\text { Rockwell \& } \\
\text { Burnell, } \\
73^{\circ} \mathrm{F}\end{array}$ & $\begin{array}{l}\text { Specific } \\
\text { Gravity }\end{array}$ & $\begin{array}{l}\text { Tensile } \\
\text { Modulus of } \\
\text { Elasticity, } \\
73^{\circ} \mathrm{F} \text { (PSI) }\end{array}$ & $\begin{array}{l}\text { Flexural } \\
\text { Modulus of } \\
\text { Elasticity, } \\
73^{\circ} \mathrm{F} \text { (PSI) }\end{array}$ \\
\hline ABS & 0.3 & R105 & 1.05 & 236,000 & 266,000 \\
\hline $\mathrm{PC}$ & 0.2 & $\mathrm{R} 115$ & 1.20 & 290,000 & 310,000 \\
\hline ABS-PC & - & R110 & 1.20 & 265,000 & 270,000 \\
\hline
\end{tabular}


The printed propeller blade geometry can also be used to cast a mold. Using this method, propellers can be manufactured from hard polyurethane, or other substance which affords greater loads to be subjected upon the propeller blades both in prototype testing and for use on underwater vehicles. The additional benefit to constructing a mold is that it is more economical than using an FDM machine, and multiple molds can be made, decreasing the manufacturing time.

The three propeller blades with their connecting attachments took an estimated $91 \mathrm{hrs}$ $4 \mathrm{~min}$ to print. The printing process at 29 hours is shown in Fig. 3-4. In order to reduce both time and cost spent, the blades were printed with the female side of the connection to the hub facing upwards, which decreased the amount of FDM support structure.

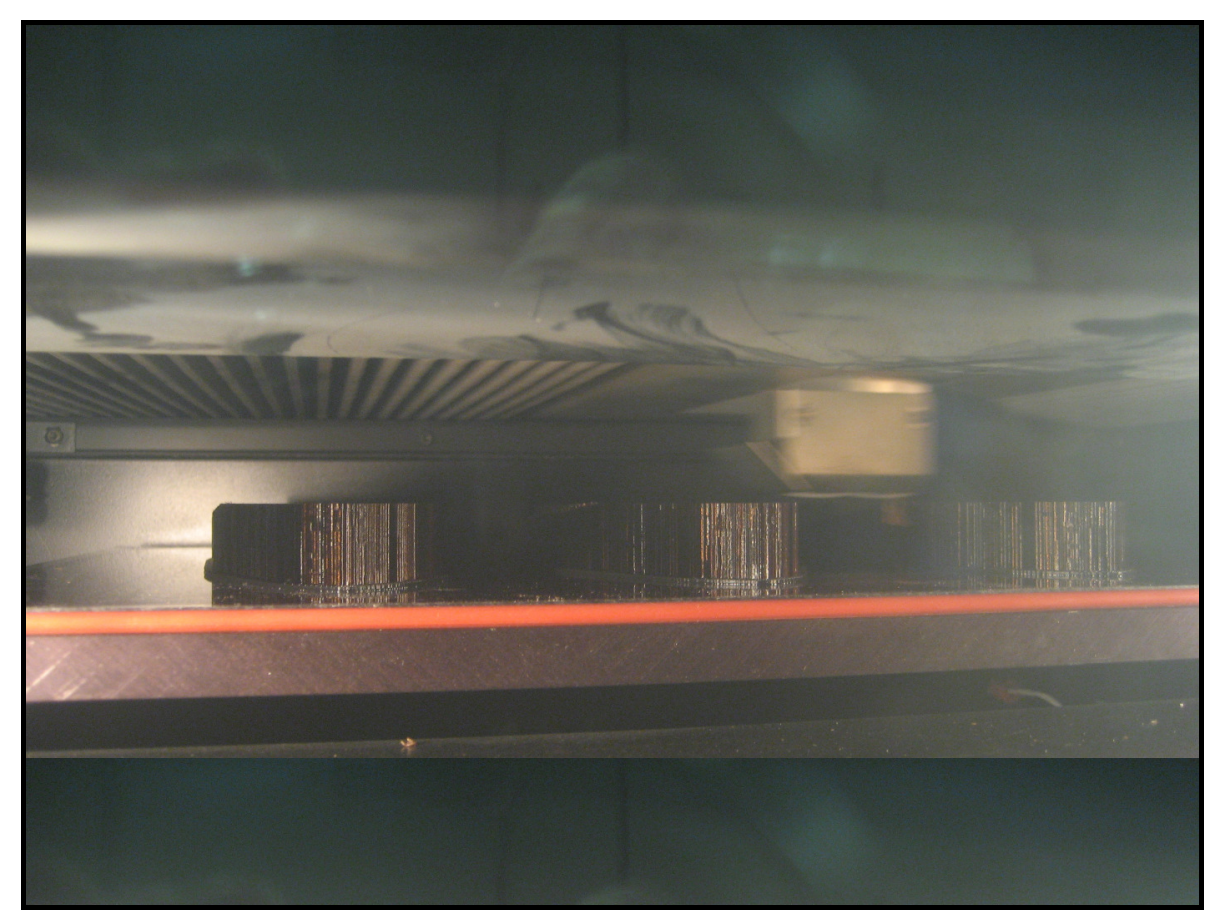

Figure 3-4: FDM printing at 29 hours.

Once the propeller blades are printed, the support structure is dissolved in a chemical bath of soluble concentrate ${ }^{3}$. The bath containment vessel also produces vibrations, which aid in the removal of the support structure. After the propeller parts are removed from the bath and

\footnotetext{
${ }^{3}$ The chemical bath used was WaterWorks soluble concentrate P400SC, in a one part concentrate to eleven parts water ratio.
} 
dried, the blades require smoothing. A combination of sandpaper and filler primer can achieve a smooth finish ${ }^{4}$. The primer fills in the gaps among the composite material threads used by the FDM printer for blade production.

\footnotetext{
${ }^{4}$ Dupli-Color high-build formula primer can be used.
} 


\section{Studies on an Off-The-Shelf Propeller for the SeaBED class AUV ${ }^{5}$}

The effectiveness of off-the-shelf propellers was determined experimentally in this case study. The propellers were tested to match their desired operational vehicle speed while maintaining speeds appropriate to their thruster pairings. The Bollard test was used for the purpose of testing how great a thrust the propeller could produce for a given propeller blade RPM while at rest. The mobile cart test was used to simulate the propeller thrust capacity at operational speeds.

The vehicle modeled in this case study was a low-speed, high-drag underwater vehicle. Desired thruster rotational speed ranged from 60 - 120 Revolutions Per Minute (RPM). Cosmos FloWorks ${ }^{\mathrm{TM}}$, a fluid-dynamic software, calculated the vehicle's necessary thrust for a single propeller at a design speed of $1 \mathrm{~m} / \mathrm{s}$. From the design point of total thrust required at 1 $\mathrm{m} / \mathrm{s}$, the thrust needed for other points can be extrapolated from the relationship Drag $\propto$ Velocity $^{3}$, as in Fig. 4-1 [13].

\footnotetext{
${ }^{5}$ The SeaBED AUV is one of several in its class developed in the Deep Submergence Lab at the Woods Hole Oceanographic Institute.
} 


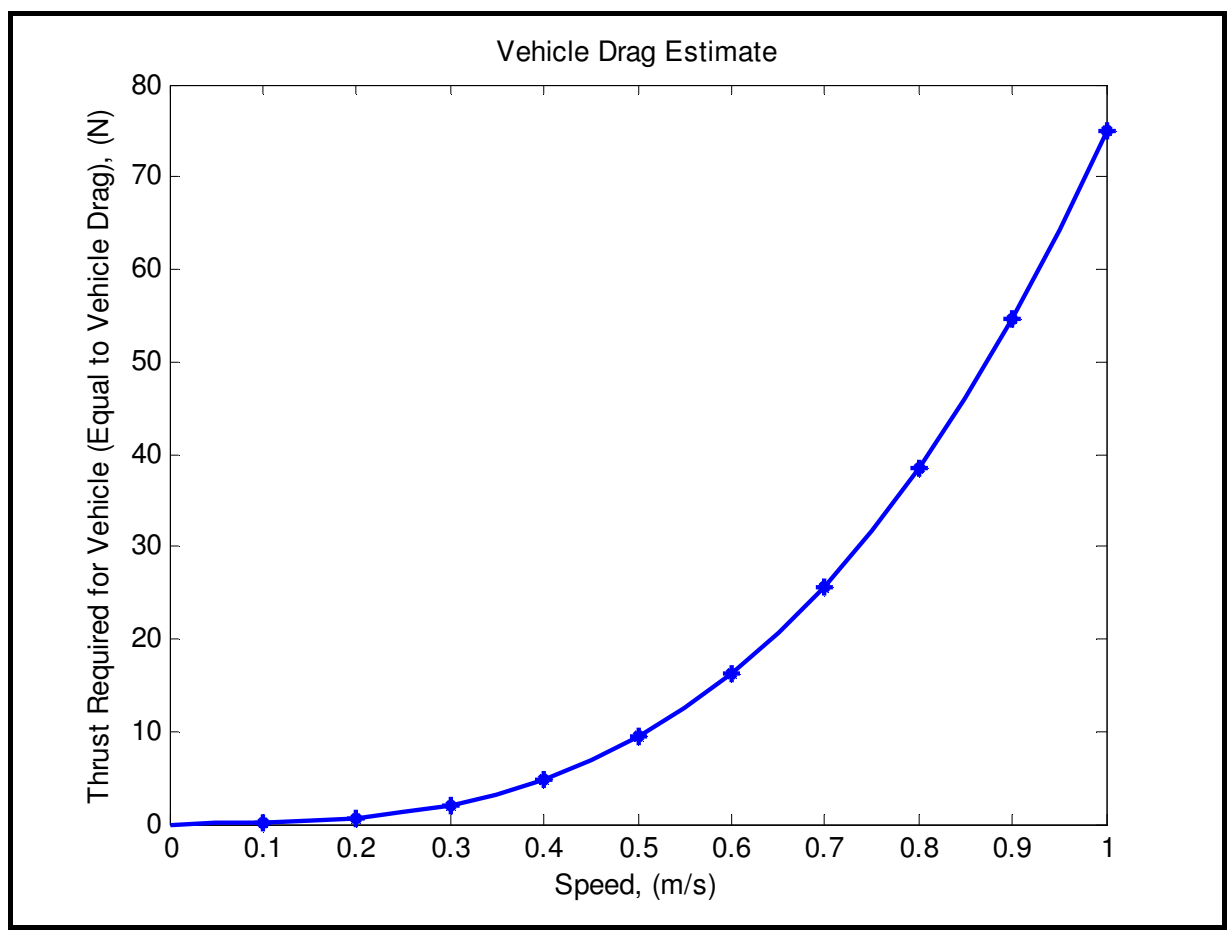

Figure 4-1: Required thrust estimate for a low-speed, high-drag AUV.

\subsection{Experimental Set-up}

The 20m tow-tank at Woods Hole Oceanographic Institute was used for experimentation. The majority of the set-up was on the tow-tank carriage platform (Fig. 4-2). 


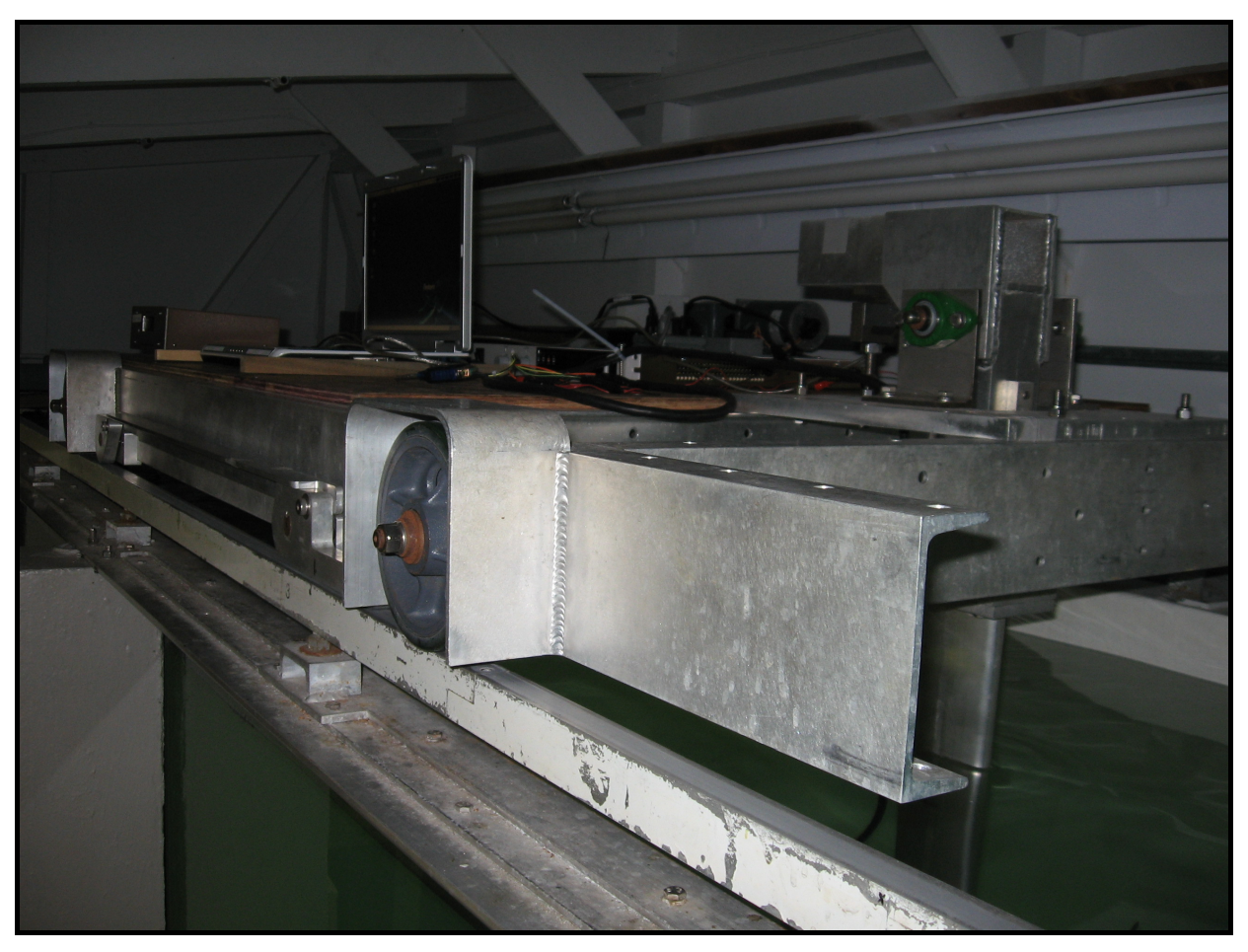

Figure 4-2: Tow-tank carriage.

The following instruments were secured to the top of the carriage:

- Laptop computer

- Power supply

- Load cell

- Thruster mount with thruster and propeller attached

The computer used a Linux program for recording the voltage and current draw of the thruster motor at a specified RPM. The power supply was set at $48 \mathrm{~V}$, and was monitored for possible spikes in current when first powered. The load cell was attached in compression to the top of the thruster mount, and measured the thrust force of the propeller. The thruster mount used a foil shape to minimize external effects of turbulent flow on the testing of the propeller. The propeller and thruster attached underneath the thruster mount using three hose clamps (Fig. 4-3). 


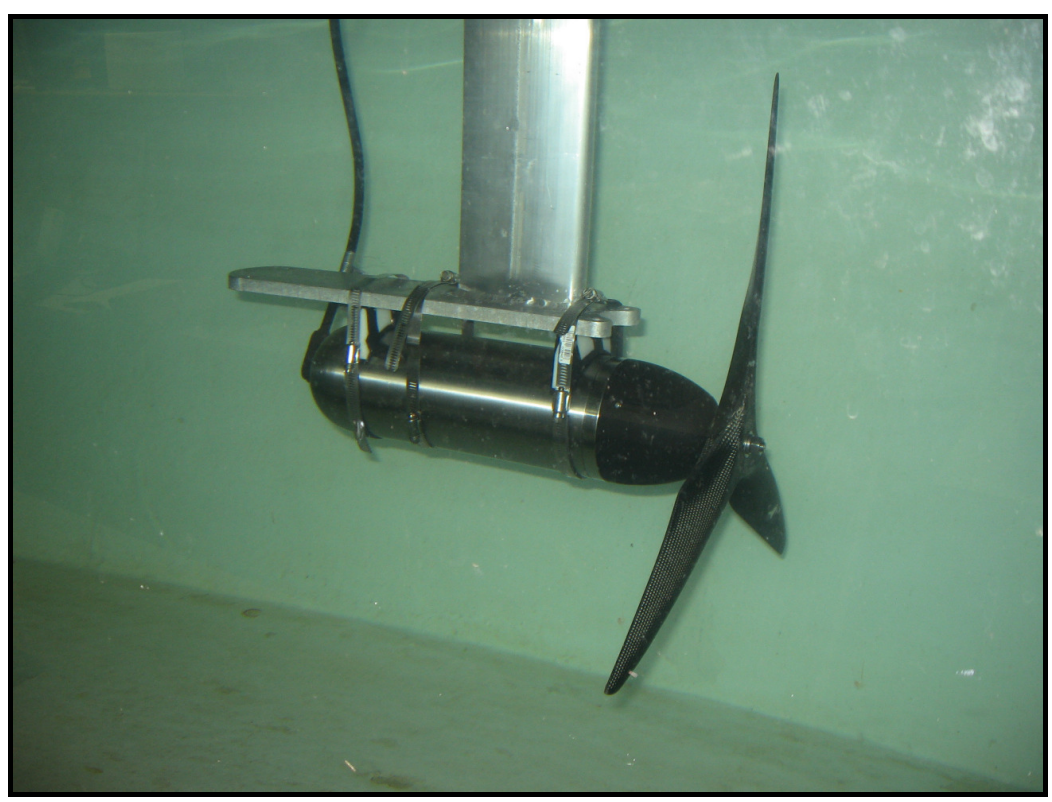

Figure 4-3: Thruster mount.

The computer used a program which controlled propeller RPM while recording the thruster current and voltage over the time of the test run.

\subsection{Method for Bollard and Mobile Cart Test Analysis}

A set range of data values was averaged, so that the data analyzed was taken at the same time for all runs. This simplifies the process, and eliminates data that could be skewed towards the end of the run. A brief analysis showed the current and voltage remained fairly constant throughout a run, making the process of truncating and averaging data a good measurement of current and voltage.

The force measurement recorded was taken during the middle of the run, at the point at which the cart had gained full momentum, but before it had started to slow down. The cart was programmed such that the cart would accelerate and decelerate to and from the desired speed linearly, for a desired distance. Both desired cart speed and total distance of the run were input into the cart's computer. Propeller rotation values ranged from 16.65RPM to 133.2RPM, and for these values, current and voltage were recorded by machine. 


\subsection{Bollard Test Results}

The Bollard test results are shown in Fig. 4-4 and 4-5. The tow-tank testing provides a comparison among different variables that are independent of RPM, including: current, A, (Amps), voltage, $\mathrm{V}$ (Volts) and thrust $(\mathrm{N})$, as in Fig. 4-4. Fig. 4-5 compares the propeller thrust $(\mathrm{N})$, the propeller rotational speed (RPM) and the power input to the thruster motors $(\mathrm{J} / \mathrm{s})$.

As expected, the propeller voltage remained close to the set voltage, at $\sim 48$ Volts. However, the current draw acted as an indicator of the upper end of the propeller's performance. The current draw was at (O)0.1 Amps for the lower propeller speeds tested, and increased exponentially for the highest speeds, to (O)1. As propeller rotational speed increased, thrust values increased exponentially, with a maximum value of thrust recorded as $64.5 \mathrm{~N}$ at a propeller speed of $133 \mathrm{RPM}$. Numerical values for the Bollard Test and the Moving Cart Test can be found in Appendix B.4. Fig. 4-5 provides an estimate of the power consumption required by the propellers in order to achieve propeller speeds from 0-133 RPM.

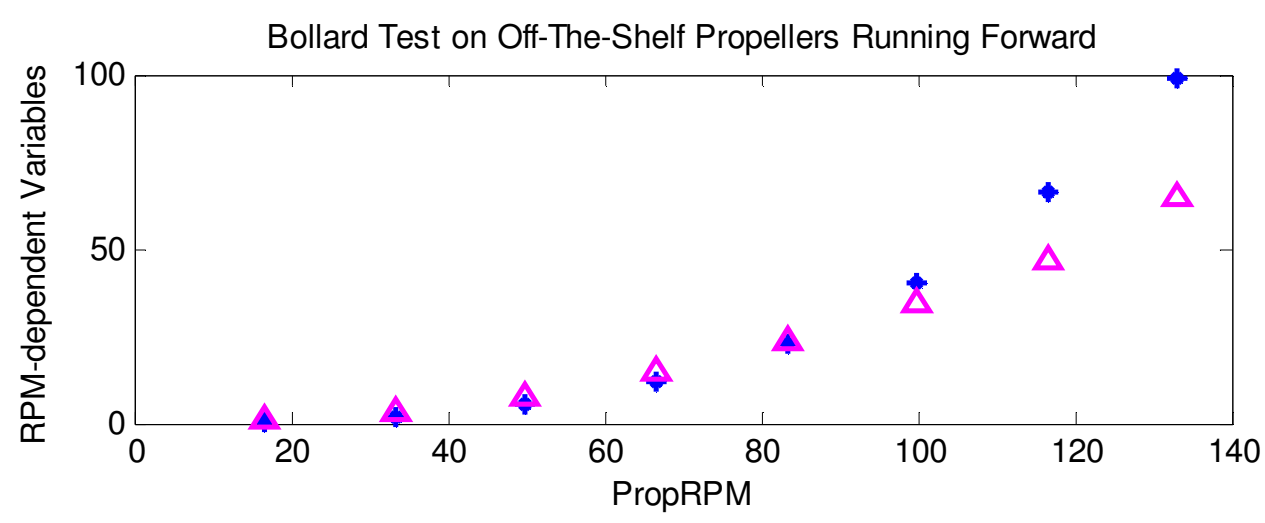

Bollard Test on Off-The-Shelf Propellers in Reverse

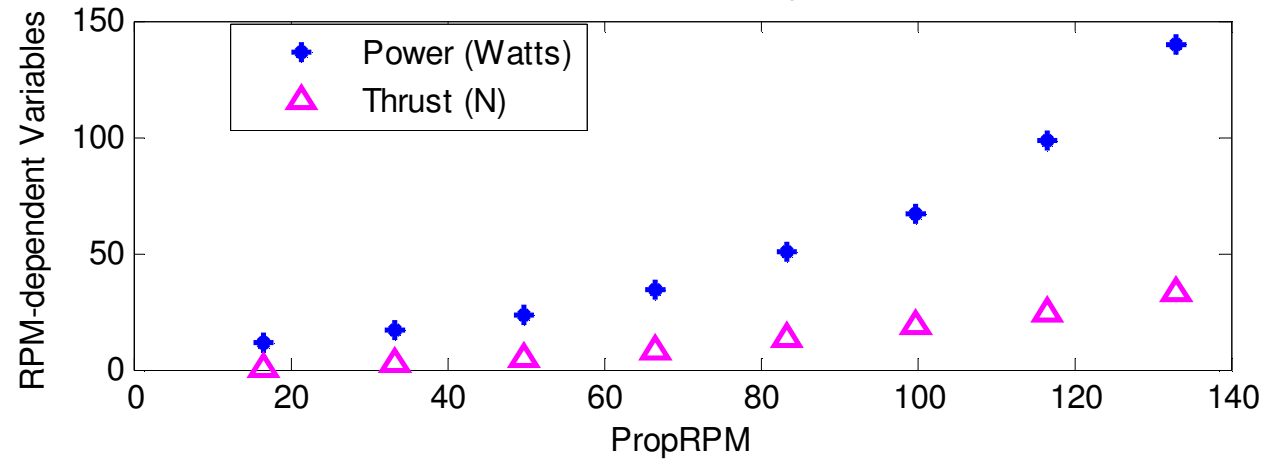

Figure 4-4: Off-the-shelf propeller Bollard Test results. 

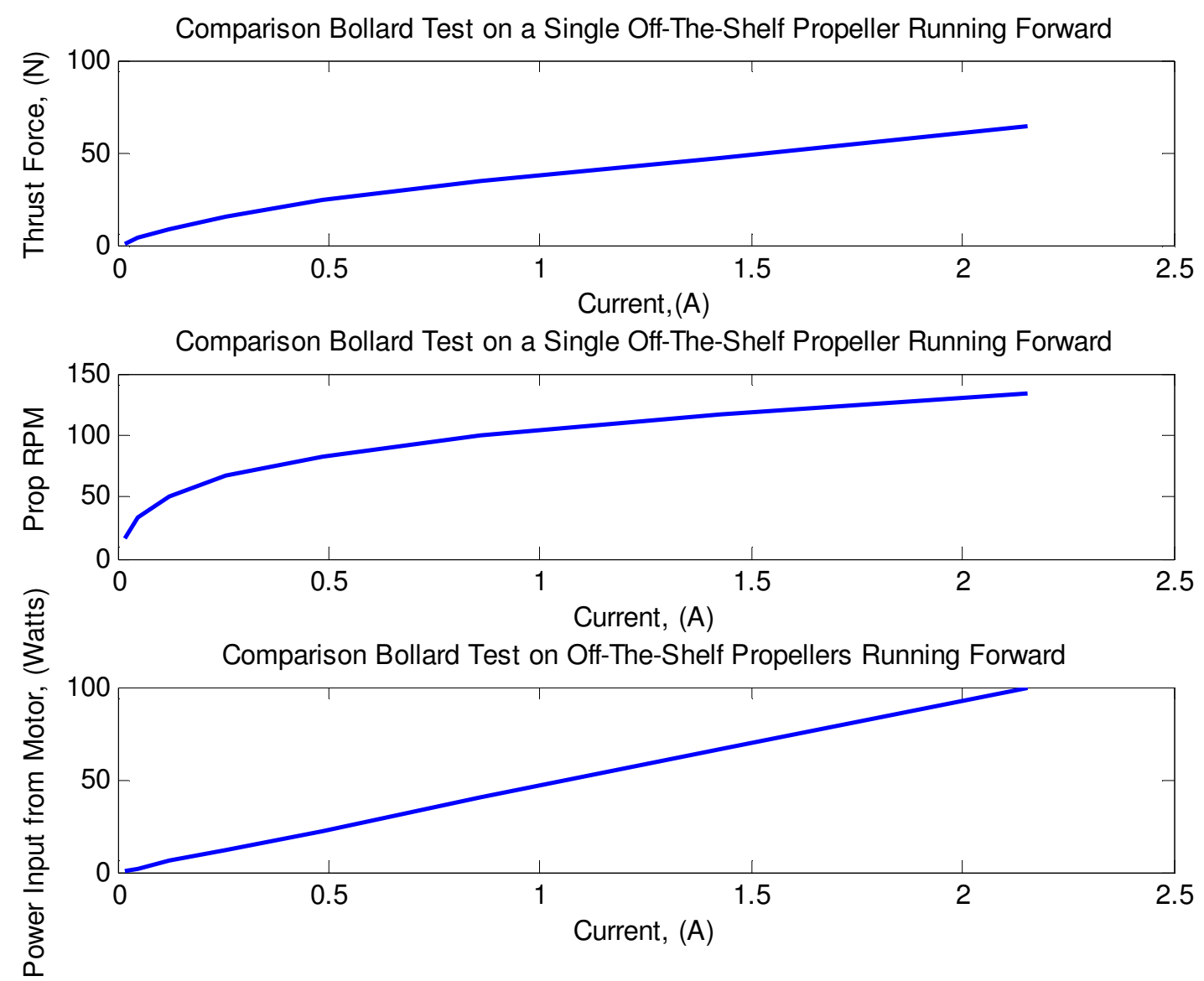

Figure 4-5: Bollard Test comparison of thrust, propeller RPM and power input with respect to current.

\subsection{Mobile Cart Results}

The mobile cart tests compare the propeller thruster current and voltage intake and the thrust force produced, as summarized in Fig. 4-6. Each diagonal line of data for the thrust corresponds to a set propeller RPM. The voltage remained at a constant $48 \mathrm{~V}$ throughout the test runs, while the current increased exponentially from $(0) 0.1$ to $(0) 1$. The maximum thrust occurred at a cart-simulated speed of $0.25 \mathrm{~m} / \mathrm{s}$, with a value of $61.88 \mathrm{~N}$ for a propeller rotational speed of 133 RPM. The current, and therefore power requirements, increase significantly for rotational values greater than the 99.9 RPM test series (Appendix B.4). Fig. 4-6 super-imposes the thrust requirements shown in Fig. 4-1, to clearly present the tested propeller's range of operation. Fig. 4-7 summarizes the off-the-shelf propeller performance using $K_{T} v s$. $J$ curves. 


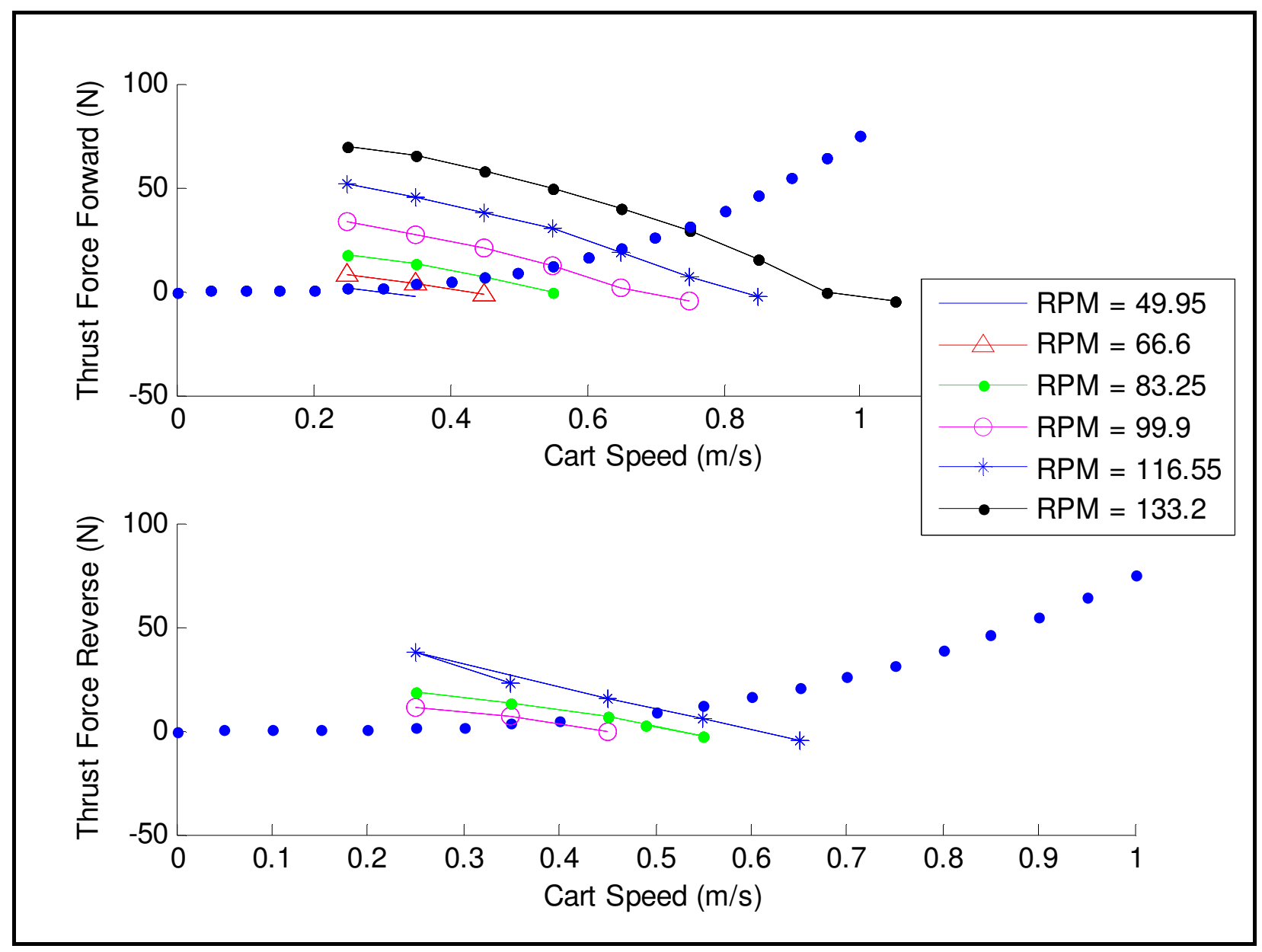

Figure 4-6: Moving Cart Test results. All data ABOVE the dotted line meet the calculated drag requirements for the vehicle. 


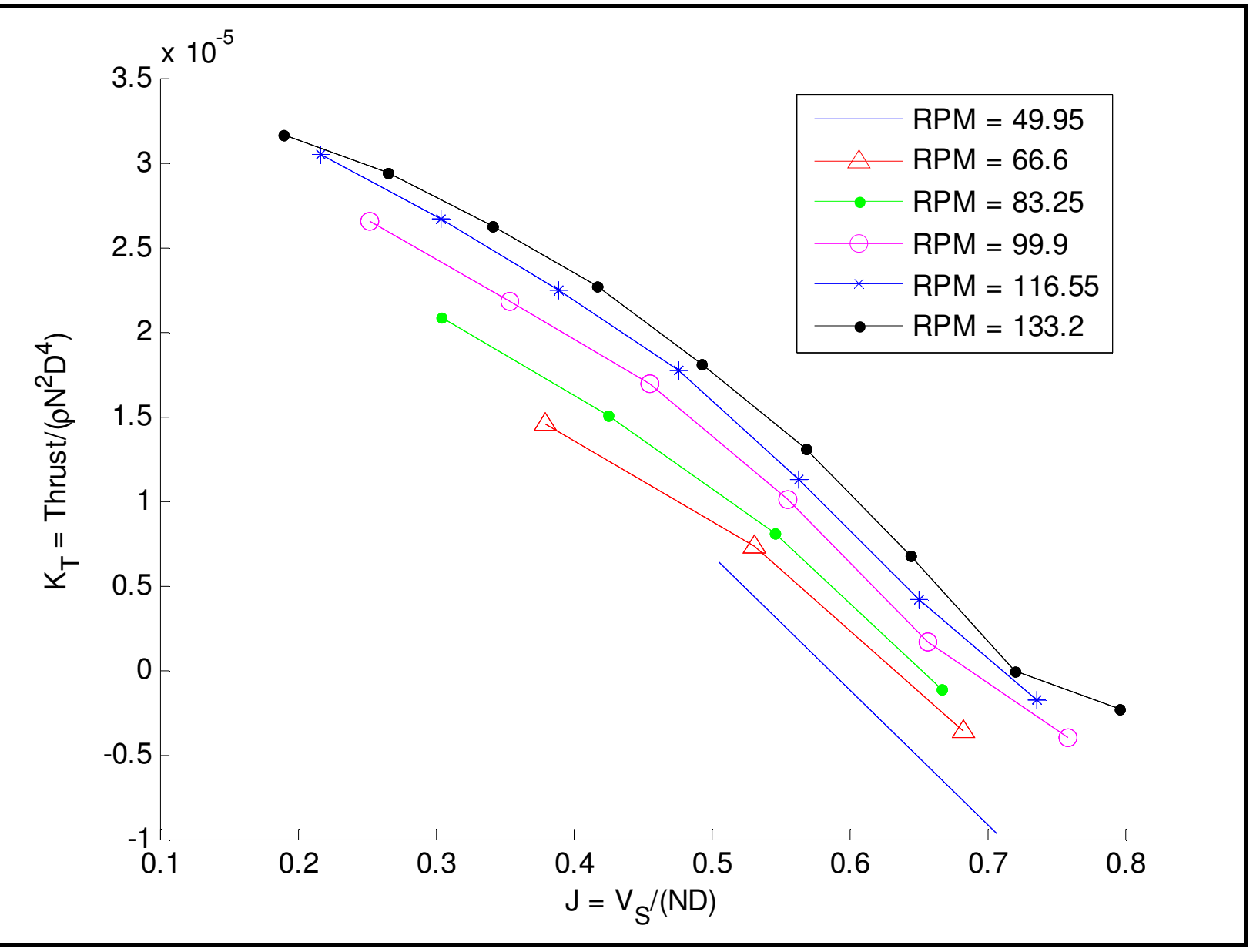

Figure 4-7: Off-the-shelf propeller forward Mobile Cart test results, thrust coefficient, $\mathbf{K}_{T}$ vs. the advance coefficient, $\mathbf{J}$.

The value of maximum speed for the forward-moving propeller must not compromise the propeller thruster limitations or exceed reasonable power requirements. Therefore, the highest speed point of interest is that which satisfies propeller speed, power and thrust requirements at $0.55 \mathrm{~m} / \mathrm{s}$ for forward propulsion and at $0.35 \mathrm{~m} / \mathrm{s}$ for reverse. Overall, the thruster tests indicate that the propeller does not meet requirements for a design speed of $1 \mathrm{~m} / \mathrm{s}$. Therefore, a propeller will be designed and produced to meet the vehicle operational requirements. A detailed description of this AUV propeller design is presented as a case study in Chapter 5. 


\section{Case Study: Designing and Producing a SeaBED Class AUV Propeller}

Vehicles carrying large quantities of instrumentation, moving at relatively slow speeds fall under the low-speed, high-drag category of AUVs. The main purpose of the study is to create a propeller blade for optimal performance for the design characteristics of a test case AUV. Another object of this research is to determine the effects of $C_{L}$ on an off-the-shelf propeller design. ${ }^{6}$ OpenPVL and basicPVL provide the means to analyze the required design characteristics of such an AUV, as shown in the remainder of this chapter. In this study the design codes were used to:

- Estimate the performance of the off-the-shelf propeller.

- Design a custom propeller for the vehicle.

User input parameters can be derived from an off-the-shelf propeller design, and the code used to estimate the performance of these propellers. Thus, the propeller design tool can be used to guide the designer to an appropriate off the shelf design.

\subsection{Test Case AUV Characteristics}

The off-the-shelf propeller used in the test-case AUV was modeled for efficiency, and it had the following characteristics:

- Tests showed that the propeller operated on this vehicle at 170RPM in order to reach a speed of $1.0 \mathrm{~m} / \mathrm{s}$, and at 60RPM for a speed of $0.3 \mathrm{~m} / \mathrm{s}$. Since the desired range of RPS was roughly between 1-3, 2 RPS was be taken as the target rotational speed.

- The propeller had three blades, or $z=3$.

- The hub was $0.031 \mathrm{~m}$, and was 0.0104 in terms of $r / R$.

\footnotetext{
${ }^{6}$ The 'off-the-shelf' propeller used by the AUV in this study is a 24in. carbon fiber model airplane propeller developed by Aircraft International Carbon Propellers.
} 
- The diameter, D, was 0.594 .

- The chordlength distributions, $c / D$ with respect to $r / R$, where $r$ was the location on the radius and $R$ was the radius length, were measured using calipers, as in Table B-1.

- Due to the placement and size of the thrusters, the axial flow ratio was equal to one for this particular AUV.

To validate the propeller design process, a test vehicle was used. Test vehicle characteristics implemented in the propeller design included [13,14]:

- Design speed, Vs, was $1 \mathrm{~m} / \mathrm{s}$.

- Inflow wake velocity variation, Vvar $=/-0.03 \mathrm{~m} / \mathrm{s}$.

- Design depth was $200 \mathrm{~m}$ to $1 \mathrm{~km}$.

- Cavitation effects were tested for a depth of $d=2 \mathrm{~m}$.

- Propeller type was assumed free tip double screw.

- The required forward thrust at operational cruising speed for the AUV was $150 \mathrm{~N}$, or $75 \mathrm{~N}$ per propeller.

- Tests taken from thrusters of a previous AUV show that it was most acceptable to model the propeller at 2RPS.

- Thruster diameter was small relative to the propeller diameter, at $4.25 \mathrm{in}$. or $0.1080 \mathrm{~m}$, and was ignored.

- Hull length, $L=96$ in., or $2.438 m$.

\subsection{Blade Selection}

The number of blades used on the propeller was chosen by varying parameters of the blade number, diameter, and revolutions per second in $\mathrm{m}$-file basicPVL.m. The blade numbers analyzed ranged from 3 to 6 . The diameter was varied from 0.5 to $0.9 \mathrm{~m}$, since the values of the advance coefficient, $\mathrm{J}$, were within an acceptable range of 0.3 to 1.9. Rotational speeds appropriate for the analyzed thruster range from 2 to 3 RPS. The results were assessed using enumerated contour plots, which were produced for each blade number, and varied contours of the efficiency $\eta$, with the ranges of diameter, $D$ (in the $y$-axis), and revolutions per second (RPS), N (in the x-axis). Figure 5-1 shows a contour plot for the chosen number of blades, 3. Plots for 4, 5 and 6 blades can be found in Appendix B.3. 


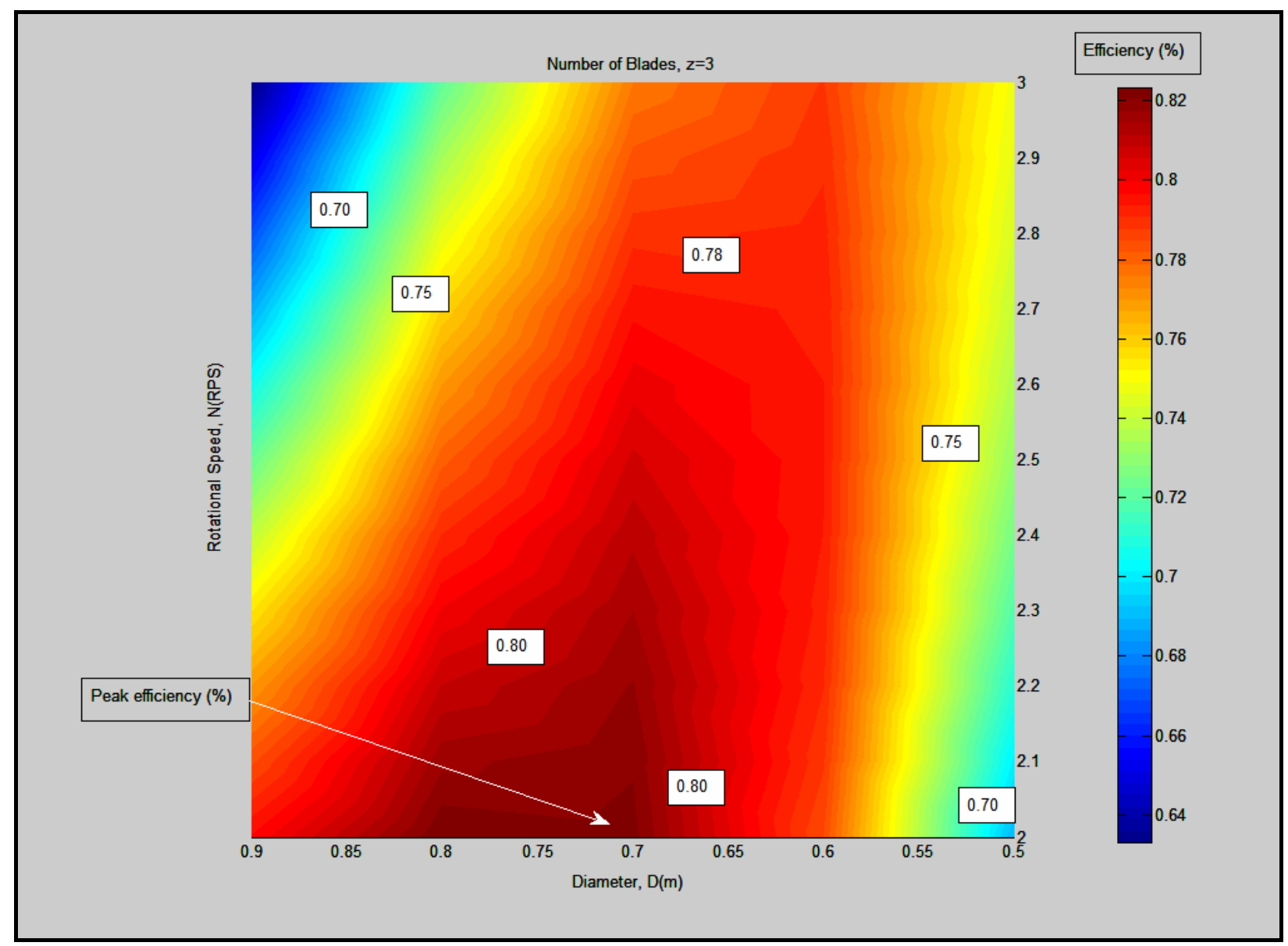

Figure 5-1: Efficiency for a 3-bladed propeller.

The initial design exhibited three blades, since a three-bladed propeller is more efficient in all ranges of diameter and rotational speed than the others. This is because a larger number of blades with a smaller diameter provide greater efficiency for the lowest range of $\mathrm{N}$, whereas the three-bladed propeller provides the most efficiency at 2 RPS, which coincides with the design speed $1.0 \mathrm{~m} / \mathrm{s}$ [14]. Furthermore, the three-bladed propeller had a wide range of high efficiency ( $70 \%$ or more) at all ranges of desirable values for $\mathrm{N}$. It is interesting to note that the maximum efficiency on the 3-blade propeller was for smaller values of $\mathrm{N}$. The blade chosen that best satisfies all the desirable values of $\mathrm{N}$, is $0.625 \mathrm{~m}$ in diameter, with an initial calculated efficiency of 0.7091 . 


\subsection{Propeller Performance Characteristics Comparison}

A comparison between both the initial and final propeller design was made. This helped determine how to improve the existing propeller, as well as to provide guidance on purchasing future off-the-shelf propellers. Detailed blade performance characteristics and blade geometry are shown in figures 5-1 and 5-2 respectively. Figure 5-3 and 5-4 show the performance and geometry for the equivalent off-the-shelf type propeller. Note that the off-the-shelf equivalent propeller required section lift coefficients of about 1.0 which is close to stall and would have high drag. Therefore, the analysis indicates that the off-the-shelf propeller is overloaded for this application. It is important to note that the table values used optimal calculations for the pitch over diameter, and these values were significantly different from the actual $P / D$, which is measured as 0.427 for $r / R$ equal to 0.7 . The data for the final propeller design, in which lift and non-cavitation requirements were easily fulfilled, is shown in Table 5-1.

Table 5-1. Final Optimized Propeller Performance vs. Radius Position

$\begin{array}{cccccccr}r / R & \mathrm{~V}^{*} & \mathrm{~B} & \mathrm{Bi} & \mathrm{C}_{\mathrm{L}} & \mathrm{G} & \Sigma & -\mathrm{C}_{\mathrm{Pmin}} \\ 0.2000 & 1.2788 & 51.8538 & 57.9439 & 0.4936 & 0.0228 & 43.7157 & 0.56 \\ 0.2500 & 1.4060 & 45.5278 & 51.9462 & 0.4871 & 0.0239 & 118.7362 & 0.55 \\ 0.3000 & 1.5478 & 40.3256 & 46.7912 & 0.4867 & 0.0258 & 97.8371 & 0.53 \\ 0.4000 & 1.8621 & 32.4814 & 38.6047 & 0.4866 & 0.0293 & 67.4235 & 0.49 \\ 0.5000 & 2.2023 & 26.9896 & 32.5685 & 0.4754 & 0.0314 & 48.0748 & 0.48 \\ 0.6000 & 2.5581 & 22.9968 & 28.0254 & 0.4599 & 0.0317 & 35.5383 & 0.46 \\ 0.7000 & 2.9242 & 19.9909 & 24.2683 & 0.4075 & 0.0293 & 27.1253 & 0.38 \\ 0.8000 & 3.2973 & 17.6570 & 20.8232 & 0.3519 & 0.0235 & 21.2771 & 0.33 \\ 0.9000 & 3.6749 & 15.7984 & 17.5931 & 0.3120 & 0.0146 & 17.0847 & 0.27 \\ 0.9500 & 3.8645 & 15.0050 & 16.0380 & 0.2578 & 0.0091 & 15.4287 & 0.24 \\ 1.0000 & 4.0543 & 14.2864 & 14.5165 & 0.2391 & 0.0005 & 13.9991 & 0.22\end{array}$

In this design, the rake/D and the skew were assumed to be zero. The ratio of pitch to diameter, P/D, was calculated using Equation 5.1 [4]:

$$
P / D=\tan \left(\mathrm{B}_{i}+\alpha_{i}\right) \pi\left(\frac{r}{R}\right), \text { where } \alpha_{i}=1.54^{\circ}, \text { from NACA Mean Line } \mathrm{a}=0.8 . \quad 5.1
$$

The design requirements are summarized into Tables 5-2, 5-3 and 5-4, from which the optimized propeller was fabricated. 
Table 5-2: Blade geometry for optimized propeller, using ML type NACA, $a=0.8$, and NACA-66 sections.

$\begin{array}{llllllll}\mathbf{r} / \mathbf{R} & \mathbf{P} / \mathbf{D} & \mathbf{C} / \mathbf{D} & \mathbf{\Delta} \boldsymbol{\alpha} & \mathbf{f o} / \mathbf{C} & \text { to/c } & \text { rake/D } & \text { Skew } \\ 0.2000 & 1.0660 & 0.2269 & 2.6878 & 0.0335 & 0.079 & 0 & 0 \\ 0.2500 & 1.0609 & 0.2193 & 2.4448 & 0.0331 & 0.078 & 0 & 0 \\ 0.3000 & 1.0590 & 0.2148 & 2.2207 & 0.0330 & 0.070 & 0 & 0 \\ 0.4000 & 1.0599 & 0.2029 & 1.8460 & 0.0330 & 0.055 & 0 & 0 \\ 0.5000 & 1.0638 & 0.1885 & 1.5609 & 0.0323 & 0.050 & 0 & 0 \\ 0.6000 & 1.0693 & 0.1693 & 1.3438 & 0.0312 & 0.046 & 0 & 0 \\ 0.7000 & 1.0635 & 0.1545 & 1.1756 & 0.0277 & 0.041 & 0 & 0 \\ 0.8000 & 1.0340 & 0.1275 & 1.0426 & 0.0239 & 0.039 & 0 & 0 \\ 0.9000 & 0.9809 & 0.0801 & 0.9355 & 0.0212 & 0.030 & 0 & 0 \\ 0.9500 & 0.9455 & 0.0572 & 0.8896 & 0.0175 & 0.025 & 0 & 0 \\ 1.0000 & 0.9042 & 0.0032 & 0.8479 & 0.0162 & 0.023 & 0 & 0\end{array}$

Table 5-3: Off-the-shelf propeller performance vs. radius position.

$\begin{array}{llllllll}\mathrm{r} / \mathrm{R} & \mathrm{V}^{*} & \mathrm{~B} & \mathrm{Bi} & \mathrm{C}_{\mathrm{L}} & \mathrm{G} & \Sigma & -\mathrm{C}_{P \min } \\ 0.2000 & 1.0888 & 68.7862 & 73.2997 & 1.0691 & 0.0143 & 198.7506 & 0.93 \\ 0.2500 & 1.1767 & 59.1563 & 65.2233 & 1.2170 & 0.0169 & 169.9533 & 1.02 \\ 0.3000 & 1.2923 & 51.1250 & 58.0726 & 1.3675 & 0.0211 & 140.6989 & 1.12 \\ 0.4000 & 1.5825 & 39.2448 & 46.5828 & 1.3573 & 0.0289 & 93.5663 & 1.09 \\ 0.5000 & 1.9215 & 31.3387 & 38.2319 & 1.3741 & 0.0342 & 63.2903 & 1.05 \\ 0.6000 & 2.2877 & 25.8917 & 32.1280 & 1.3561 & 0.0365 & 44.5251 & 1.02 \\ 0.7000 & 2.6704 & 21.9756 & 27.2771 & 1.2697 & 0.0349 & 32.5871 & 0.95 \\ 0.8000 & 3.0637 & 19.0490 & 23.0115 & 1.1103 & 0.0289 & 24.6877 & 0.81 \\ 0.9000 & 3.4634 & 16.7904 & 19.1435 & 0.8699 & 0.0185 & 19.2633 & 0.63 \\ 0.9500 & 3.6646 & 15.8456 & 17.3195 & 0.6695 & 0.0117 & 17.1820 & 0.49 \\ 1.000 & 3.8661 & 14.9993 & 15.5565 & 0.6255 & 0.0007 & 15.4153 & 0.46\end{array}$

Table 5-4: Blade geometry for the off-the-shelf propeller, using ML type NACA, $a=0.8$, and NACA-66 sections.

$\begin{array}{llllllll}\mathrm{r} / \mathrm{R} & \mathrm{P} / \mathrm{D} & \mathrm{c} / \mathrm{D} & \Delta \alpha & \text { fo/c } & \text { to/c } & \text { rake/D } & \text { Skew } \\ 0.2000 & 1.2058 & 0.0770 & 3.1565 & 0.0726 & 0.067 & 0 & 0 \\ 0.2500 & 1.1707 & 0.0742 & 2.9210 & 0.0826 & 0.061 & 0 & 0 \\ 0.3000 & 1.1572 & 0.0751 & 2.6597 & 0.0929 & 0.053 & 0 & 0 \\ 0.4000 & 1.1494 & 0.0846 & 2.1720 & 0.0922 & 0.047 & 0 & 0 \\ 0.5000 & 1.1505 & 0.0814 & 1.7890 & 0.0933 & 0.037 & 0 & 0 \\ 0.6000 & 1.1551 & 0.0739 & 1.5026 & 0.0921 & 0.032 & 0 & 0 \\ 0.7000 & 1.1476 & 0.0647 & 1.2873 & 0.0862 & 0.028 & 0 & 0 \\ 0.8000 & 1.1137 & 0.0533 & 1.1221 & 0.0754 & 0.023 & 0 & 0 \\ 0.9000 & 1.0532 & 0.0386 & 0.9926 & 0.0591 & 0.021 & 0 & 0 \\ 0.9500 & 1.0130 & 0.0301 & 0.9381 & 0.0455 & 0.019 & 0 & 0 \\ 1.0000 & 0.9663 & 0.0017 & 0.8892 & 0.0425 & 0.018 & 0 & 0\end{array}$


It is very important to note that the actual pitch over diameter ratio for the existing propeller was about a third of the optimal pitch calculated for blades of its geometry. The 2D analysis determined that the lift coefficients on the existing propeller were above 1.0 for about 85 percent of the length the of propeller's radius.

\subsection{Off-the-Shelf Propeller Analysis and Improvement}

Lift coefficients above 1.0 may result in stalling effects, and therefore are unacceptably high. Therefore, appropriate analysis included determining the effect of hub diameter variation on the lift coefficients, while still considering the effect of hub variation on propeller efficiency, as shown in Fig. 5-2. Several assumptions in this study are of note: the efficiency does not consider the possibility for lost performance due to stalling from high lift coefficients. Additionally, the performance modeling assumes a NACA camber, thickness, and a calculated pitch to diameter $(\mathrm{P} / \mathrm{D})$ ratio.
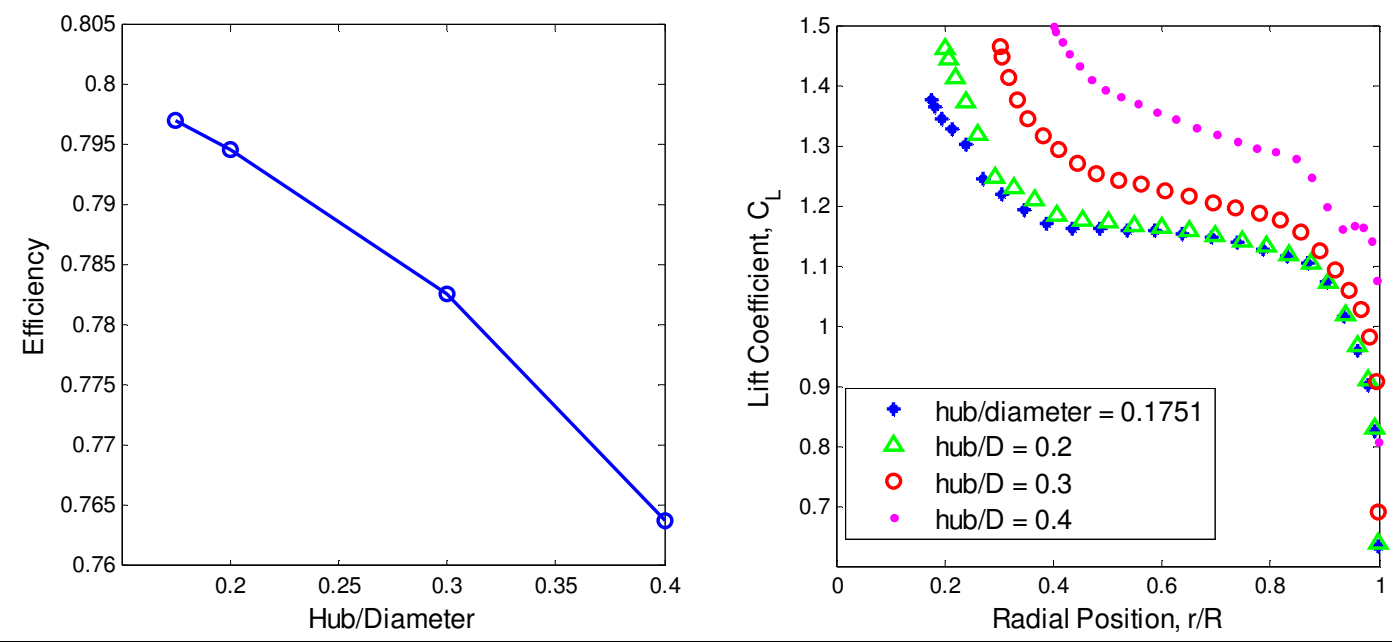

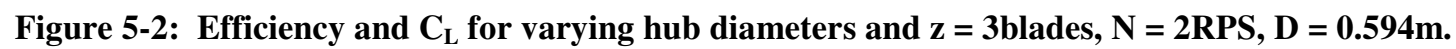

One experimental problem for this study was to determine whether adding a fairing to increase hub size would be performance enhancing. From Fig. 5-2, it is clear that the efficiency decreased with an increase in the hub radius. As the hub size increased, so did the lift coefficient; attaching a fairing to reduce the effects of stalling was not applicable in this case. Therefore, the propeller geometry must increase in diameter or chordlength to reduce the lift coefficients enough to avoid stalling. 
Due to high values of lift coefficients for the model-airplane propeller, the effect of different blade diameters on lift coefficient and calculated efficiency were modeled. A list of criteria determined the chosen propeller design:

- The lift coefficient would be reduced to 0.7 or below at the hub, and below 0.25 at the blade tips.

- The efficiency would be maximized for $z=3$ and $N=2$, with the original chord distribution.

- The optimal diameter would be as close as possible to the original diameter, while satisfying the design requirements.

The chordlength distribution which satisfied these requirements can be found using basicPVL. The numerical values for the chordlength distribution is in Appendix B.1.

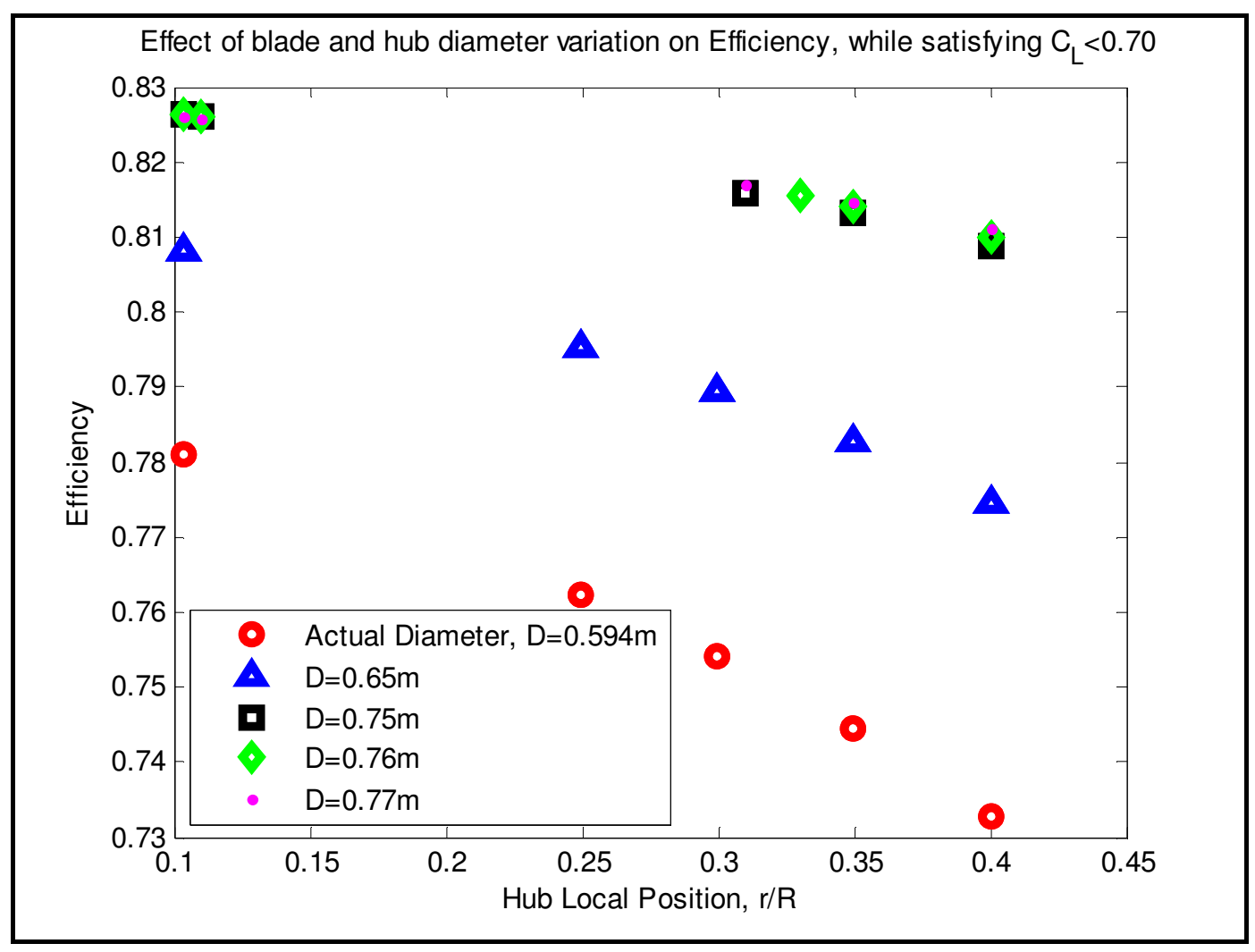

Figure 5-3: Efficiency vs. variation of blade hubs for different diameters of the off-the-shelf propeller. In this case, $C_{L}<0.7$ is satisfied for $D=0.76 \mathrm{~m}$ and $0.77 \mathrm{~m}$. 
Using the results shown in Fig. 5-3, the design chosen was $0.76 \mathrm{~m}$, with a hub extending to $r / R=0.31$, and an efficiency of 0.8154 and an actuator disk efficiency [4] of 0.9287. From Figs. 5-2 and 5-3, it is clear that an increased hub (proportionally greater than $r / R=0.2$ ) has a greater benefit for larger propeller blades (greater than $0.6 \mathrm{~m}$ ) which maintain lower rotational speeds (less than 2 RPS). Due to the relatively low RPSs needed for this vehicle and the operating depth, cavitation is not likely to occur [6]. However, the case in which cavitation could present problems will be investigated by analyzing propeller pressure coefficients at a shallow operating depth of $2 \mathrm{~m}$. A visual comparison of the off-the-shelf propeller modification and the final propeller design is presented in Figs. 5-4 and 5-5.

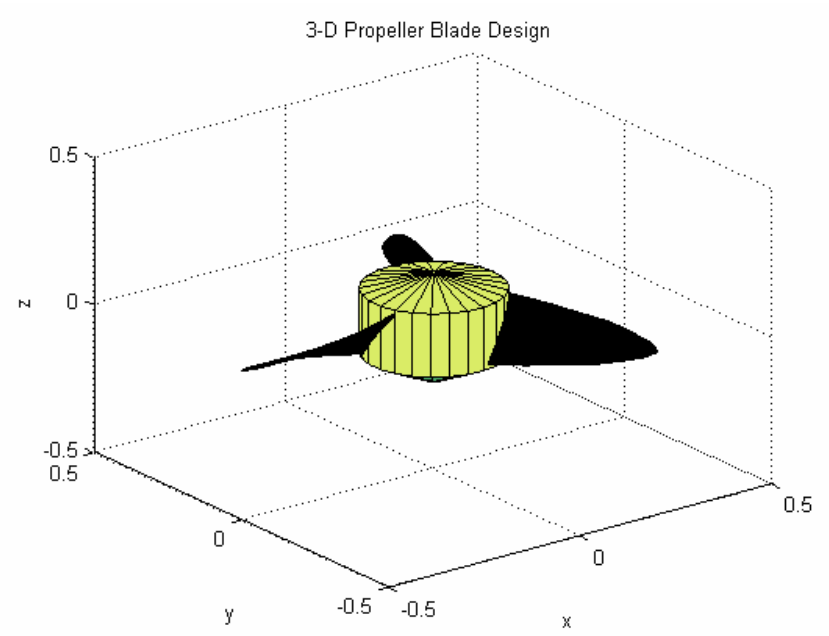

Figure 5-4: MATLAB ${ }^{\mathrm{TM}}$ representation of optimal modified off-the shelf propeller.

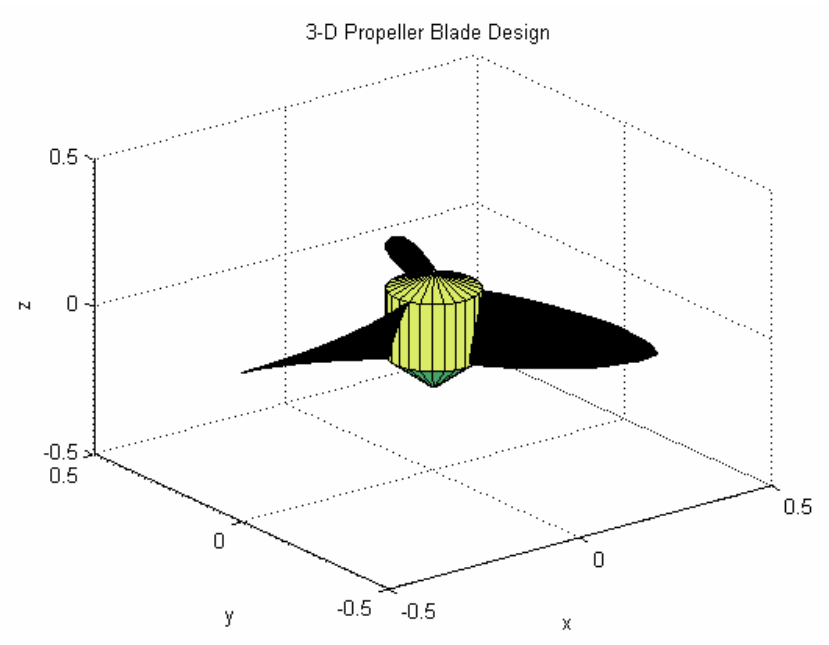


Figure 5-5: MATLAB ${ }^{\mathrm{TM}}$ representation of final propeller design.

\subsection{Propeller Hub Assembly and Shaft Design}

The hub was machined out of Delrin. The new propeller hub, (Fig. 5-6), allowed for the three individually-printed propeller blades to tightly slide into place. The unique propeller blade design allowed for the 3D printing of a propeller with a diameter that exceeded the dimensions of the FDM machine.

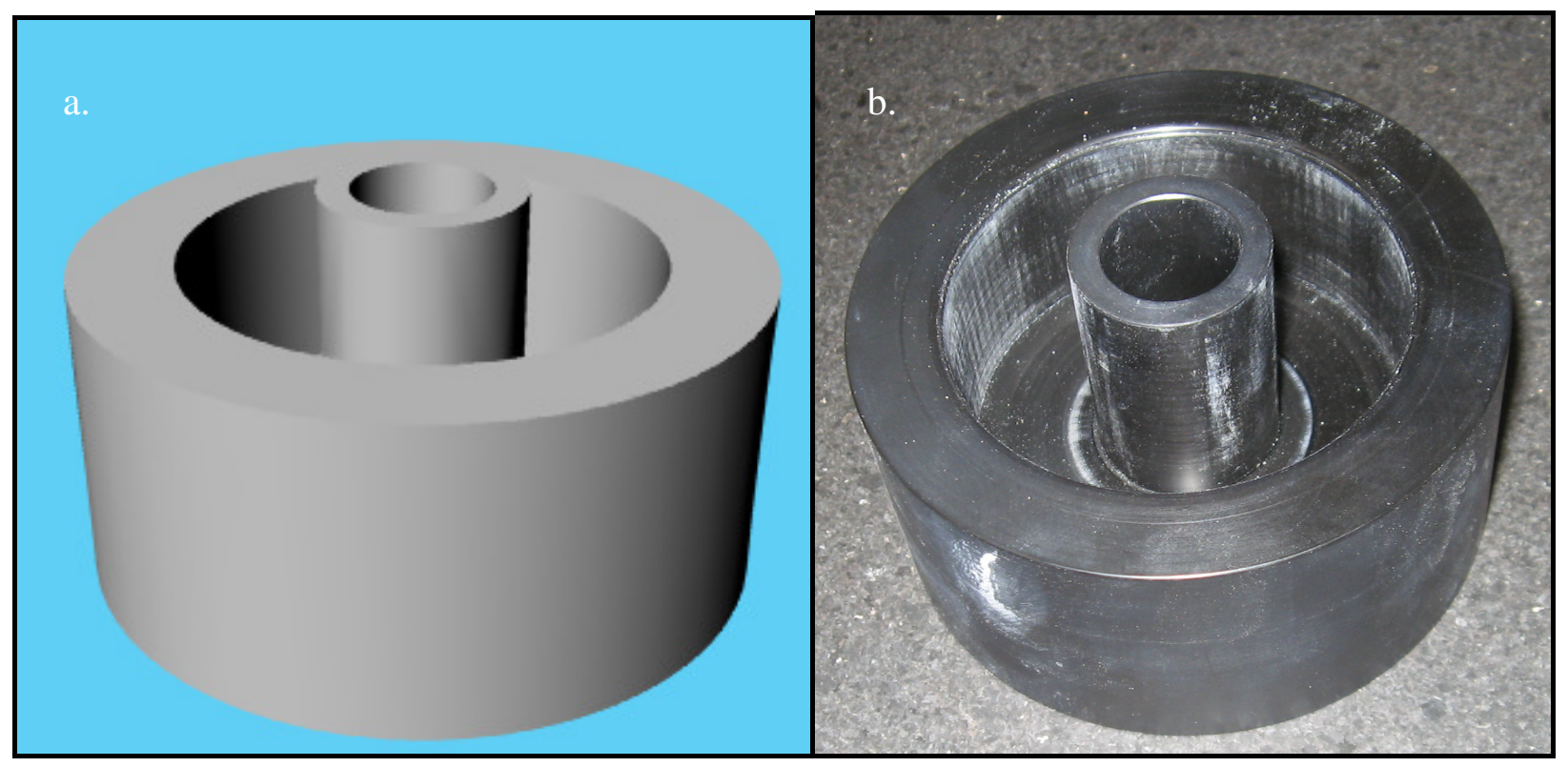

Figure 5-6: Hub design in CAD (a), and machined (b).

The propeller design is useful only if it is integrated into the design of an AUV. One of the necessary modifications is to adjust the length of the propeller shafts to accommodate the new propeller blade pitch. The purpose of the thruster shaft is to transfer the energy of the thruster motors to the propeller blades. The optimized propeller design used a pitch to diameter ratio of 1.0635 (for $r / R=0.7$ ), while the original design allowed for a propeller with blades with a lower pitch to diameter ratio of 0.427 (for $r / R=0.7$ ).

The original propeller thruster shaft was modified to work with a newer, more highlypitched propeller. The new propeller shaft design can be seen in Fig. 5-7. The shaft was machined out of Grade 5 Titanium. Dual-usability of the new shaft allowed for the new propeller to be attached using a pin and bolt with a lock-washer mechanism. For ease of test comparison 
to the off-the-shelf propellers, the shaft contained a pinhole to accommodate the width of the original propeller hub.

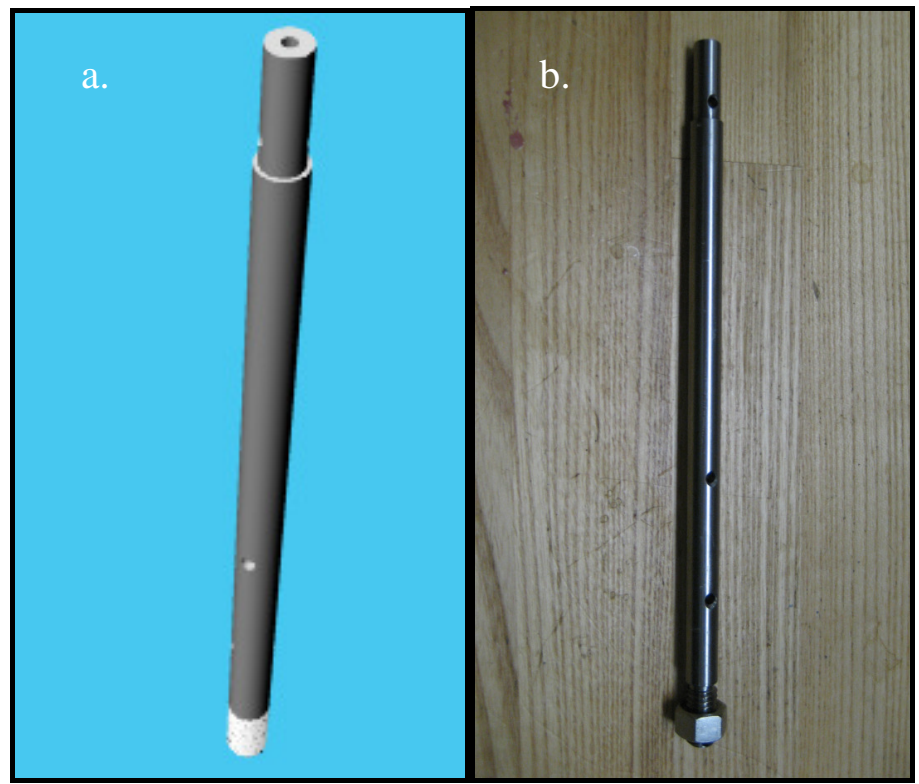

Figure 5-7: Modified thruster shaft design in CAD (a), and machined (b).

The propeller design is such that the blades can be modified and reinserted into the hub without having to manufacture a new hub for the propeller. Additionally, propeller blades can be replaced individually if necessary. The propeller assembly, including the blades, hub, hub insert and thruster shaft is shown in Figure 5-8. 


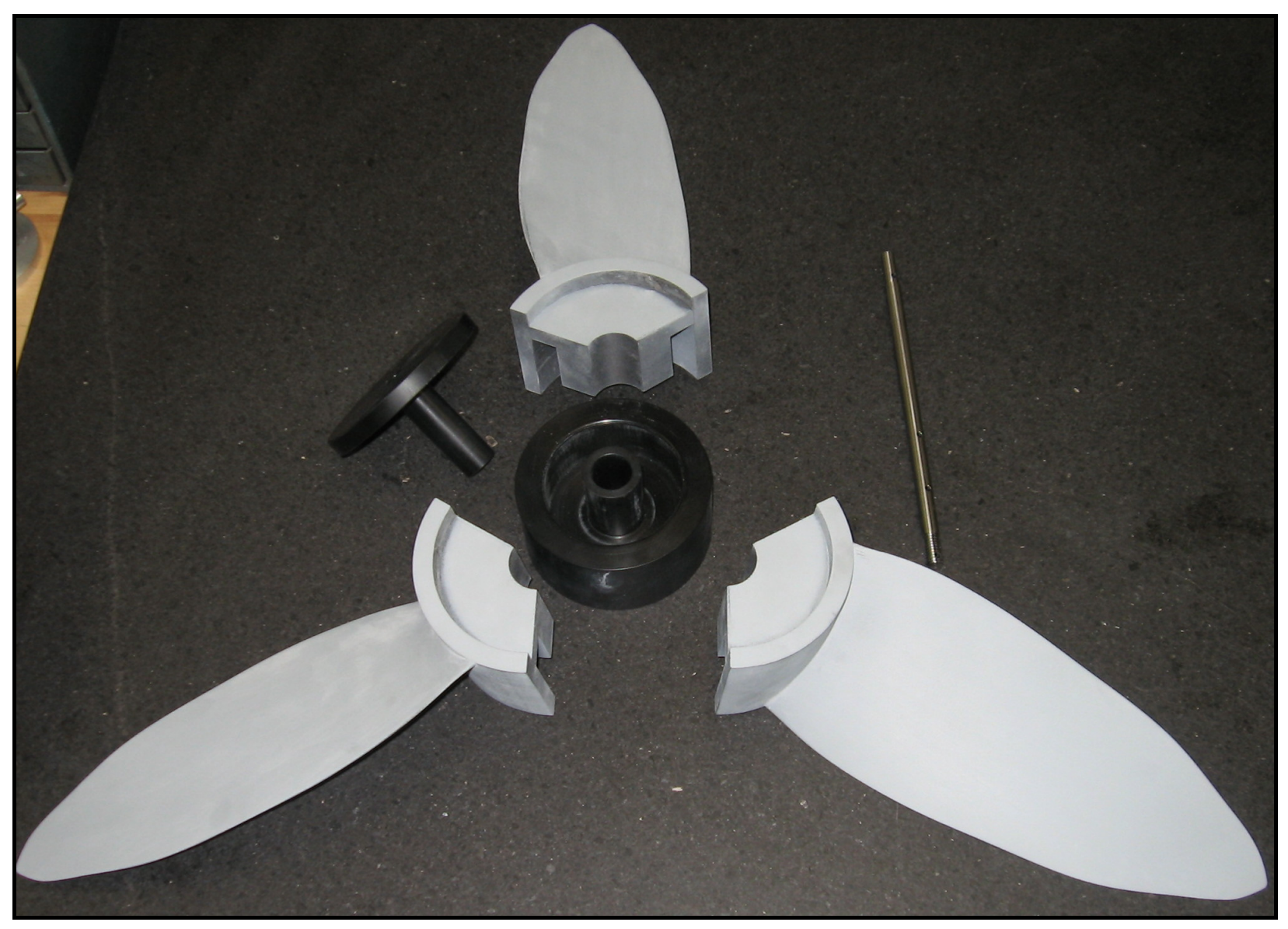

Figure 5-8: Propeller assembly.

The propeller design was validated by comparing the design propeller geometry to the FDM-printed results, as shown in Fig. 5-9. First, the propeller geometry file was exported as a $.3 \mathrm{dm}$ file. Along each chosen $\mathrm{r} / \mathrm{R}$ value five evenly-spaced points were chosen. The $3 \mathrm{D}$ printed propeller was measured on a Bridgeport milling machine using a dial indicator. Points on the surface of the propeller blade were chosen for three different $r / R$ values, $0.25,0.70$ and 0.80 . The propeller was measured in its forward and reverse positions. Figure 5-9 compares the actual blade produced to the desired blade geometry determined in MATLAB. The measurements of the actual 3D printed propeller can be found in Appendix B.5. The measurements of the blade geometry at the leading and trailing edge are erroneous due to the difficulty in pinpointing the edge of the blade with a dial indicator and effects due to deflection. However, the other points measured clearly match the desired results, as are geometrically represented in Figure 5-9. 

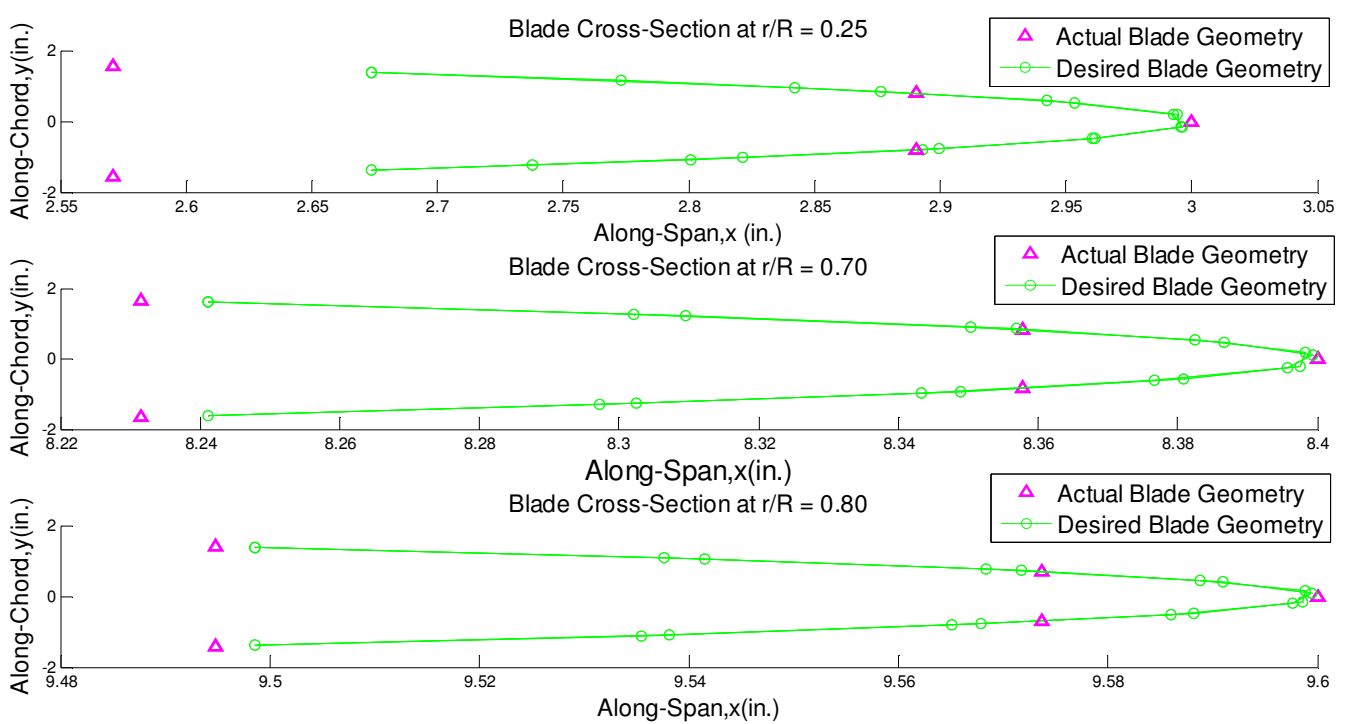

Figure 5-9: Propeller geometry validation, where the actual 3D printed blade is shown in comparison to the desired blade geometry.

\subsection{AUV Design Summary}

The final optimal propeller was designed with three blades, at 2RPS, a hub starting at $r / R(0.2)$, and a diameter of $0.6096 \mathrm{~m}$. A design depth of $2 \mathrm{~m}$ resulted in no cavitation effects. The design had a modified chord distribution which resulted in a lift coefficient ranging from less than 0.5 to less than 0.24 . The initial propeller design had an efficiency of 0.7091 , and the final design had an efficiency of 0.7642 , for an improvement of 5.51 percent from optimizing the chord distribution.

The existing propeller required attention specifically to the lift coefficient and the value of $P / D$. The PVL lifting line code does not take into consideration stalling effects of the propeller due to $C_{L}$ values greater than one. In the case of the off-the-shelf propeller analyzed, stalling effects could occur from the hub to $r / R=0.9$. Additionally, the lift coefficient values were considerably higher than that of the design propeller, and likely to cause stalling. In order to complete a rigorous analysis, efficiency losses due to high $C_{L}$ values should be determined.

The pitch over diameter value calculated for propeller performance was far lower than the actual value taken at $r / R=0.7$. Therefore, the efficiency value calculated for the existing 
propeller was erroneously dependent on an optimal circulation distribution. The P/D ratio can be increased in future blades by increasing the chordlength or decreasing the propeller diameter.

Under the assumption that the existing propeller is sold off-the-shelf for a scaled range of diameters, analysis showed rewarding performance improvements as a result of increasing both the propeller and the hub diameters. Increasing the propeller diameter for this propeller's value of $\mathrm{N}$ and geometry largely contributed its increase in calculated efficiency. The lift coefficients were within a satisfactory range for an off-the-shelf propeller increased from a $0.594 \mathrm{~m}$ to $0.76 \mathrm{~m}$ blade diameter and a hub from $r / R=0.104$ to an $r / R=0.31$. Furthermore, the efficiency showed a 3.44 percent improvement, from an efficiency of 0.7810 to 0.8154 . The efficiency improvement was actually greater than could be measured using the methods in this analysis, since the effect of stalling was not accounted for in computing the efficiency for the original propeller design. The modified propeller's $C_{L}$ values ranged from0. 6131 at the hub to 0.2839 at the tip. Consideration was made in cutting down the tips of the propeller due to their low chordlengths, but this would be counterproductive due to the fact that the modeled efficiency decreased as propeller diameter decreased, as well as the added effort and cost of modifying the propeller. The optimized design blade did have a lower calculated efficiency, however, the requirements for its range of acceptable $C_{L}$ values were more stringent, and did not account for loss of efficiency through stalling effects. If the off-the-shelf geometry were held to the range of $C_{L}$ values less than 0.5 , the propeller diameter would get prohibitively large. The original off-the-shelf propeller was unacceptable since it was overloaded, which increased the chances of stalling thus making it inefficient for its application. The performance analysis in this study showed in Table 5-3 that the unmodified off-the-shelf propeller's $C_{L}$ values were greater than 1.0 , and likely to stall.

When using the design codes, the designer must be careful to evaluate the section lift coefficients of the final design; the code does not predict the loss in performance due to stall at high lift coefficients. Also, the user input section drags would need to be increased in high lift ranges. The listing of computed lift coefficients for the blade is provided to the designer to evaluate these effects. The coefficient of lift and pitch over drag make significant contributions, and should not be overlooked. 
There are several methods that merit further investigation which would modify the existing propellers in order to provide greater efficiency. First, when a greater propeller diameter can be accommodated, the hub size of the propeller could be increased, which decreases the lift coefficient, and therefore prevents stalling. From basicPVL analysis, propellers with greater diameters show a trend of higher efficiency than other propellers. Under the assumption that the same off-the-shelf propeller geometry exists for greater propeller diameters, investigations showed that the lift coefficient, and thus overall performance, could be improved from both of these modification methods. However, larger propellers would revolve more slowly, and the efficiency of the drive shaft may suffer. Also, practical considerations generally limit the size of a propeller for a given application as well as blade strength issues.

Further investigation into optimal rotational speed will lead to better designs of AUV propellers. In this design, some approximations were made on the range of practical RPS values, due to minimal information on AUV thruster performance. A factor that was not considered, but is essential to overall AUV performance, is the interaction among the AUV's design speed, thruster speed, and power consumption. However, when detailed AUV information is available, the requirements for the propeller design will become more complex, but the same principles for the geometric design remain the same. Therefore, the MATLAB ${ }^{\mathrm{TM}}$ code and other methods used for this propeller design provide a useful tool for further applications [15]. 


\section{Case Study: OpenPVL Propeller Prototype Printable File}

OpenPVL can be used in a straightforward process to create propellers optimized for a set of necessary design characteristics. In this case, OpenPVL was used to analyze and create the geometry for a model based on the US Navy 4148 propellers. The geometry for the Navy 4148 model is listed in Appendix B.2 [16]. The propeller design was a 1:8 scale model, with characteristics which were determined by using the dimensionless thrust coefficient, $K_{T}=0.225$, and the advance coefficient, $J=0.75$. The model had three blades, and was 0.25 $\mathrm{m}$ in diameter. The propeller was designed to run in a tank that could simulate a vehicle speed of $5 \mathrm{~m} / \mathrm{s}$, and a thruster that ran at 1600 RPM.

OpenPVL created four propeller design files and two scripting files. As shown in Figure 2-5, the Single Propeller Design GUI provides a text input box for the user to create a Filename Prefix or to use the default, OpenPVL. Upon running the Single Propeller Design, the following files were saved:

- OpenPVL_Input.txt

- OpenPVL_Output.txt

- OpenPVL_Performance.txt

- OpenPVL_Geometry.dat

- OpenPVL_CADfull.txt

- OpenPVL_CADblade.txt

\subsection{OpenPVL Propeller Design Files}

The initial design characteristics were saved in an input file, as in Table 6-1. OpenPVL created files which output a summary of the propeller's design characteristics, the propeller performance, and the propeller geometry. Like MPVL, OpenPVL computed a summary of propeller design characteristics (Table 6-2) at radial increments, in addition to the design thrust coefficient and propeller efficiency, which in this case is 0.6745 [5]. The propeller performance file (Table 6-3) is useful for cavitation analysis and contains a table which lists the total inflow 
velocity $\left(\mathrm{V}^{*}\right)$, the undisturbed flow angle $(\beta)$, the hydrodynamic pitch angle $(\beta \mathrm{i})$, the vortex sheet strength (Gamma), the lift coefficient $\left(\mathrm{C}_{\mathrm{L}}\right)$, the cavitation number $(\sigma)$, and the pitch angle variation $(\Delta \alpha)$. The propeller geometry file (Table 6-4) recorded the propeller diameter, number of blades, propeller speed, hub diameter, meanline type, thickness form, pitch, skew, rake, chord distribution, camber distribution and thickness distribution. The propeller geometry files list the blade geometry which can be used to manufacture the propeller.

Table 6-1: OpenPVL Input file.

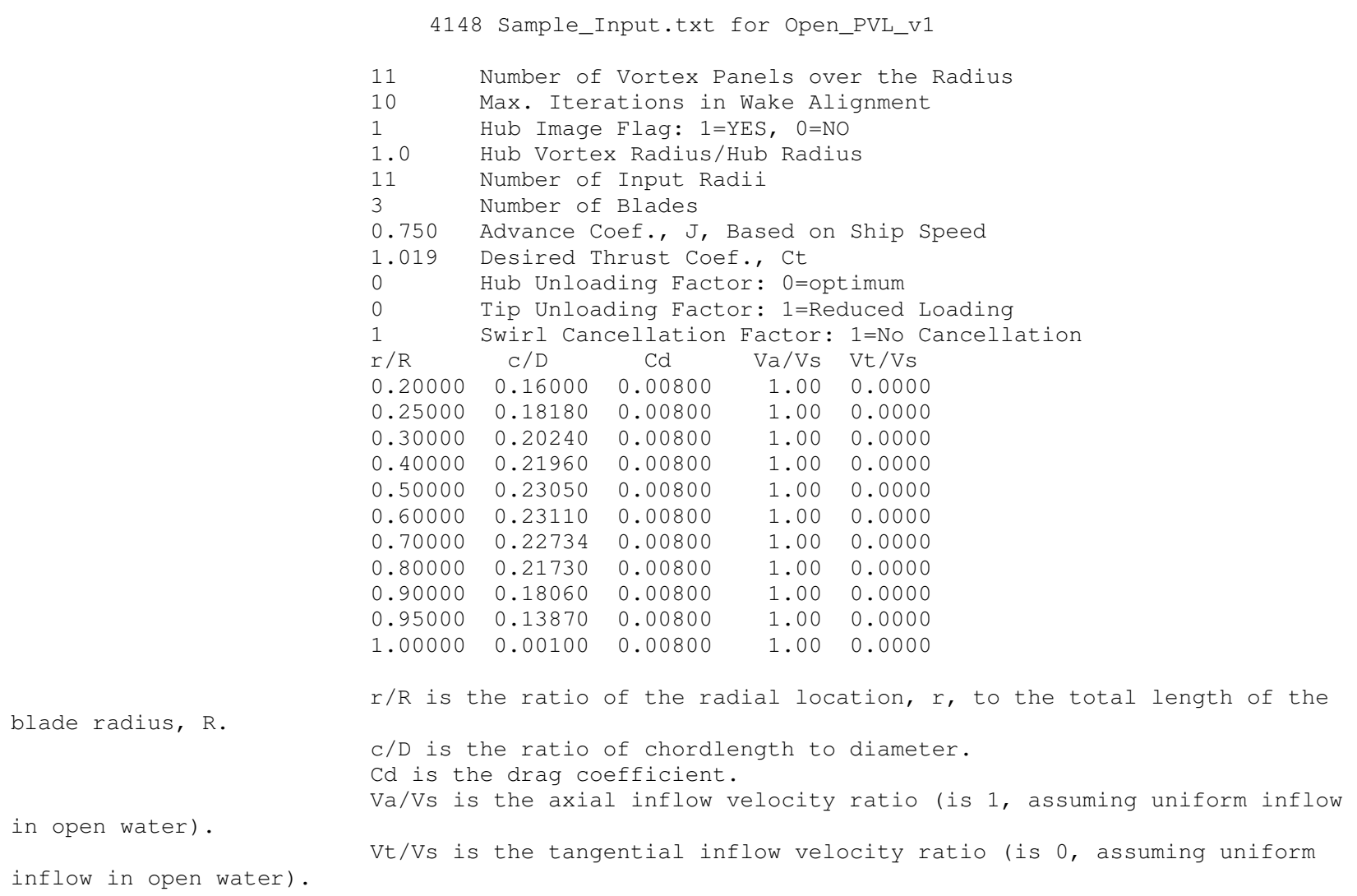


Table 6-2: OpenPVL Output file.

4148 Sample_output.txt for Open_PVL_v1

Output Table

$\mathrm{Ct}=1.0186$
$\mathrm{Cp}=1.5102$
$\mathrm{Kt}=0.2250$
$\mathrm{Kq}=0.0398$
$\mathrm{Va} / \mathrm{Vs}=1.0000$

Efficiency $=0.6745$

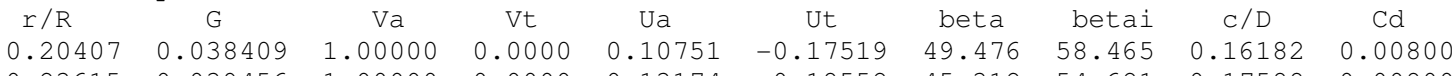

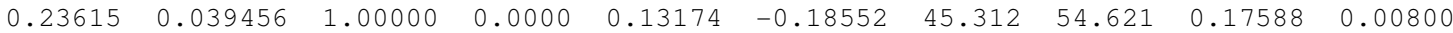

$\begin{array}{llllllllll}0.29770 & 0.043360 & 1.00000 & 0.0000 & 0.17483 & -0.19530 & 38.727 & 48.165 & 0.20179 & 0.00800\end{array}$

$\begin{array}{llllllllll}0.38374 & 0.049111 & 1.00000 & 0.0000 & 0.22444 & -0.19451 & 31.886 & 40.912 & 0.21744 & 0.00800\end{array}$

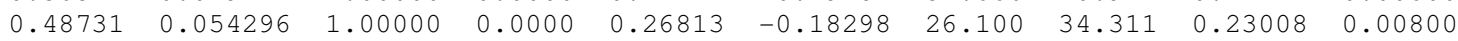

$\begin{array}{llllllllll}0.60000 & 0.056897 & 1.00000 & 0.0000 & 0.30065 & -0.16664 & 21.697 & 28.998 & 0.23110 & 0.00800\end{array}$

$\begin{array}{llllllllll}0.71269 & 0.055665 & 1.00000 & 0.0000 & 0.32274 & -0.15059 & 18.519 & 25.015 & 0.22658 & 0.00800\end{array}$

$\begin{array}{llllllllll}0.81626 & 0.049952 & 1.00000 & 0.0000 & 0.33706 & -0.13732 & 16.303 & 22.167 & 0.21412 & 0.00800\end{array}$

$\begin{array}{llllllllll}0.90230 & 0.039706 & 1.00000 & 0.0000 & 0.34600 & -0.12752 & 14.820 & 20.232 & 0.17930 & 0.00800\end{array}$

$\begin{array}{llllllllll}0.96385 & 0.025570 & 1.00000 & 0.0000 & 0.35120 & -0.12117 & 13.911 & 19.036 & 0.12112 & 0.00800\end{array}$

$\begin{array}{llllllllll}0.99593 & 0.008828 & 1.00000 & 0.0000 & 0.35358 & -0.11807 & 13.480 & 18.465 & 0.04543 & 0.00800\end{array}$

Ct is the required thrust.

$\mathrm{Cp}$ is the power coefficient. $\mathrm{Cp}=\mathrm{Cq} * 2 * \mathrm{pi} / \mathrm{J}$.

$\mathrm{Kt}$ is the thrust coefficient. Kt $=\mathrm{Ct} \mathrm{J}^{\wedge} \mathrm{J}^{\star} \mathrm{pi} / 8$, where $\mathrm{J}$ is the advance coefficient.

$\mathrm{Kq}$ is the torque coefficient. $\mathrm{Kq}=\mathrm{Cq} \mathrm{J}^{\wedge} 2 * \mathrm{pi} / 8$.

$\mathrm{Va} / \mathrm{Vs}$ is the axial inflow velocity ratio (is 1, assuming uniform inflow in open water).

Efficiency is the ratio of required thrust times the wake to the power coefficient.

$r / R$ is the ratio of the radial location, $r$, to the total length of the blade radius, $\mathrm{R}$. $G$ is the non-dimensional circulation.

$\mathrm{Va}$ is the axial inflow velocity.

Vt is the tangential inflow velocity.

Ua us the induced axial velocity.

Ut is the induced tangential velocity.

beta is the flow angle.

betaI is the hydrodynamic Pitch angle.

c/D is the ratio of chordlength to diameter.

$\mathrm{Cd}$ is the drag coefficient.

Table 6-3: OpenPVL Performance file.

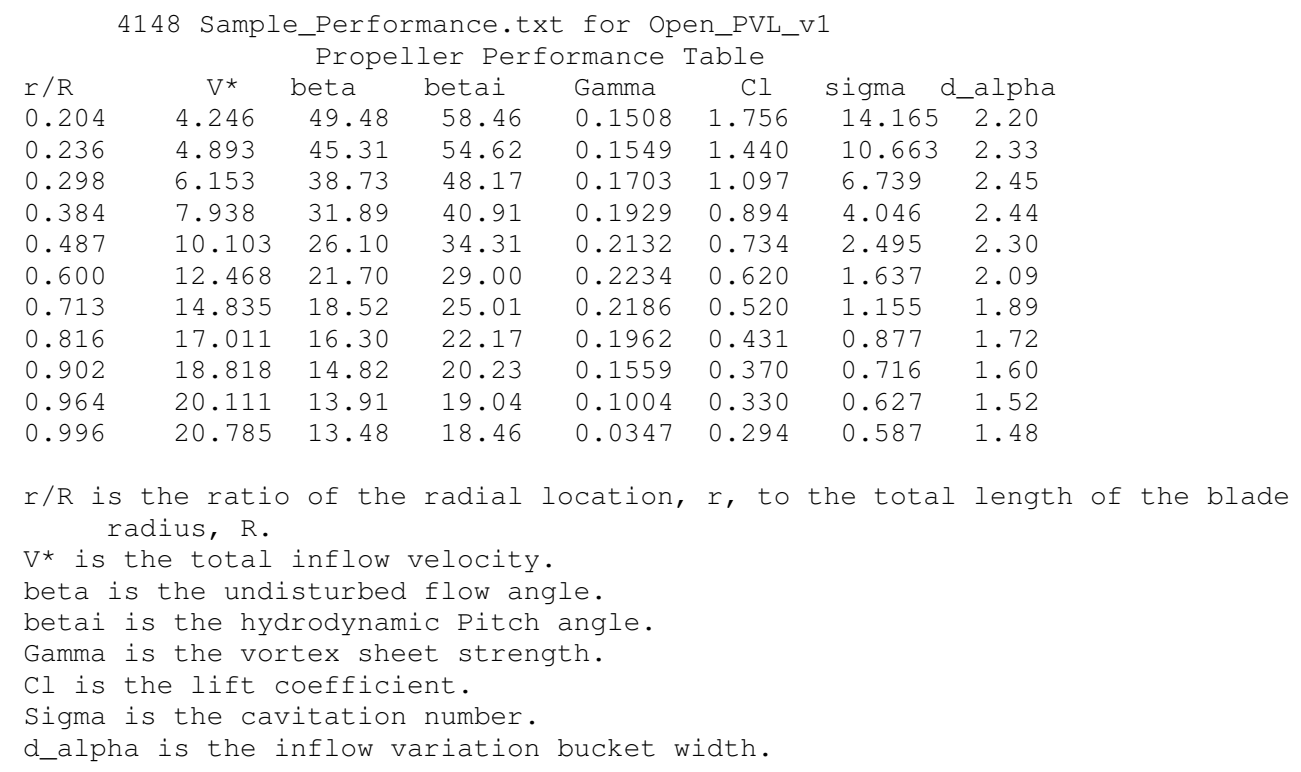


Table 6-4: OpenPVL Geometry file.

\begin{tabular}{|c|c|c|c|c|c|c|}
\hline & & & & & & \\
\hline 0.204 & 1.11 & 0.162 & 0.0310 & 0.2010 & 0.0 & 0.000 \\
\hline 0.236 & 1.11 & 0.176 & 0.0278 & 0.1676 & 0.0 & 0.000 \\
\hline 0.298 & 1.10 & 0.202 & 0.0211 & 0.1191 & 0.0 & 0.000 \\
\hline 0.384 & 1.10 & 0.217 & 0.0159 & 0.0939 & 0.0 & 0.000 \\
\hline 0.487 & 1.11 & 0.230 & 0.0117 & 0.0717 & 0.0 & 0.000 \\
\hline 0.600 & 1.11 & 0.231 & 0.0089 & 0.0541 & 0.0 & 0.000 \\
\hline 0.713 & 1.12 & 0.227 & 0.0071 & 0.0469 & 0.0 & 0.000 \\
\hline 0.816 & 1.13 & 0.214 & 0.0057 & 0.0405 & 0.0 & 0.000 \\
\hline 0.902 & 1.13 & 0.179 & 0.0046 & 0.0331 & 0.0 & 0.000 \\
\hline 0.964 & 1.14 & 0.121 & 0.0033 & 0.0288 & 0.0 & 0.000 \\
\hline 0.996 & 1.14 & 0.045 & 0.0004 & 0.0039 & 0.0 & 0.000 \\
\hline \multicolumn{7}{|c|}{$\begin{array}{l}r / \mathrm{R} \text { is the ratio of the radial location, } r \text {, to the total length of the blade } \\
\text { radius, } \mathrm{R} \text {. }\end{array}$} \\
\hline \multirow{2}{*}{\multicolumn{7}{|c|}{$\begin{array}{l}\mathrm{P} / \mathrm{D} \text { is the ratio of pitch over diameter. } \\
\mathrm{C} / \mathrm{D} \text { is the ratio of chordlength to diameter. }\end{array}$}} \\
\hline & & & & & & \\
\hline \multicolumn{7}{|c|}{$\begin{array}{l}\text { fo/C is the ratio of camber to } \\
\text { to/C is the ratio of thickness }\end{array}$} \\
\hline
\end{tabular}

\subsection{OpenPVL Geometry File into CAD}

Once the propeller was designed using OpenPVL, the MATLAB ${ }^{\mathrm{TM}}$-scripted command files, OpenPVL_CADfull.txt or OpenPVL_CADblade.txt could be read such that they produce a full propeller with the desired number of blades and a propeller blade only, respectively. The command file for the full propeller produces a blade based on the value of the height variable, and accommodating room for a fillet. In the 3D-printable design, the blades extend to 95 percent of the design hub radius, to ensure that the blade can be joined to the hub when the command file is run. An example of a RHINOTM-compatible script for the Navy 4148 based model propeller detailed in this chapter can be found in Appendix C. Depending on user propeller needs, the blade may be artificially thickened. Also, it is simple to add desired propeller features, such as fillets at the hub, and fairings, slots for pin connections, etc.

The MATLAB ${ }^{T M}$ scripting file created by OpenPVL was made specifically to provide a RHINOTM interface, although future versions of OpenPVL will be compatible with other CAD programs, such as SolidWorks ${ }^{\mathrm{TM}}$. The following are several techniques used to produce the propeller, as shown in Fig. 6-2, which was attached to a hub and filleted in RHINOTM: 
- OpenPVL chooses to extend the blade into the hub by plotting $x, y$ and $z$ dimensional values which start at 95 percent of the hub's radius and extend to the propeller's tip. Extending the blade into the hub geometry ensures that the blade and hub will be able to be joined using a Boolean Union.

- If a Boolean Union fails, consider the direction of the surface normals for the two objects you are trying to unify, using Dir.

- To create a propeller fillet, several aspects must be considered. First, the height of the hub must accommodate the fillet's radius. Second, the blade must not have a trailing edge value of zero. This creates a sharp edge, which is incompatible with the fillet command. Next, when using FilletSrf to fillet the hub to the blade surface, the fillet generally works the best when the surface normal direction is outward for both the hub and the blade.

- A propeller fairing can be built into the hub by using the paraboloid function.

- Creating a .STL file in RHINOTM requires the following procedure:

1) After all custom propeller modifications have been made, apply the command Join to all the polysurfaces. For closed surfaces, use a Boolean Union.

2) Once all the surfaces are joined, use Mesh. Ensure that the mesh settings are such that the minimum edge length exceeds the size of the strand of plastic used to fabricate the propeller. For example, the minimum edge length was set to $0.025 \mathrm{~m}$ when printed using an FDM strand size of $0.007 \mathrm{~m}$. For best results, check the boxes for refine mesh and pack textures.

3) Next, delete all of the polysurfaces to make sure the mesh has been completed for the desired portions of the propeller.

4) Use the Weld command. Select the polygon mesh surface, and choose and angle tolerance of 180 degrees.

5) Next, use the UnifyMeshNormals command. This ensures that the surface normals for the mesh object are all going in the same direction, i.e., inward or outward.

6) Finally, select SelNakedMeshEdgePt to determine that all of design is watertight. A file must be considered watertight to be saved as a .STL file. 


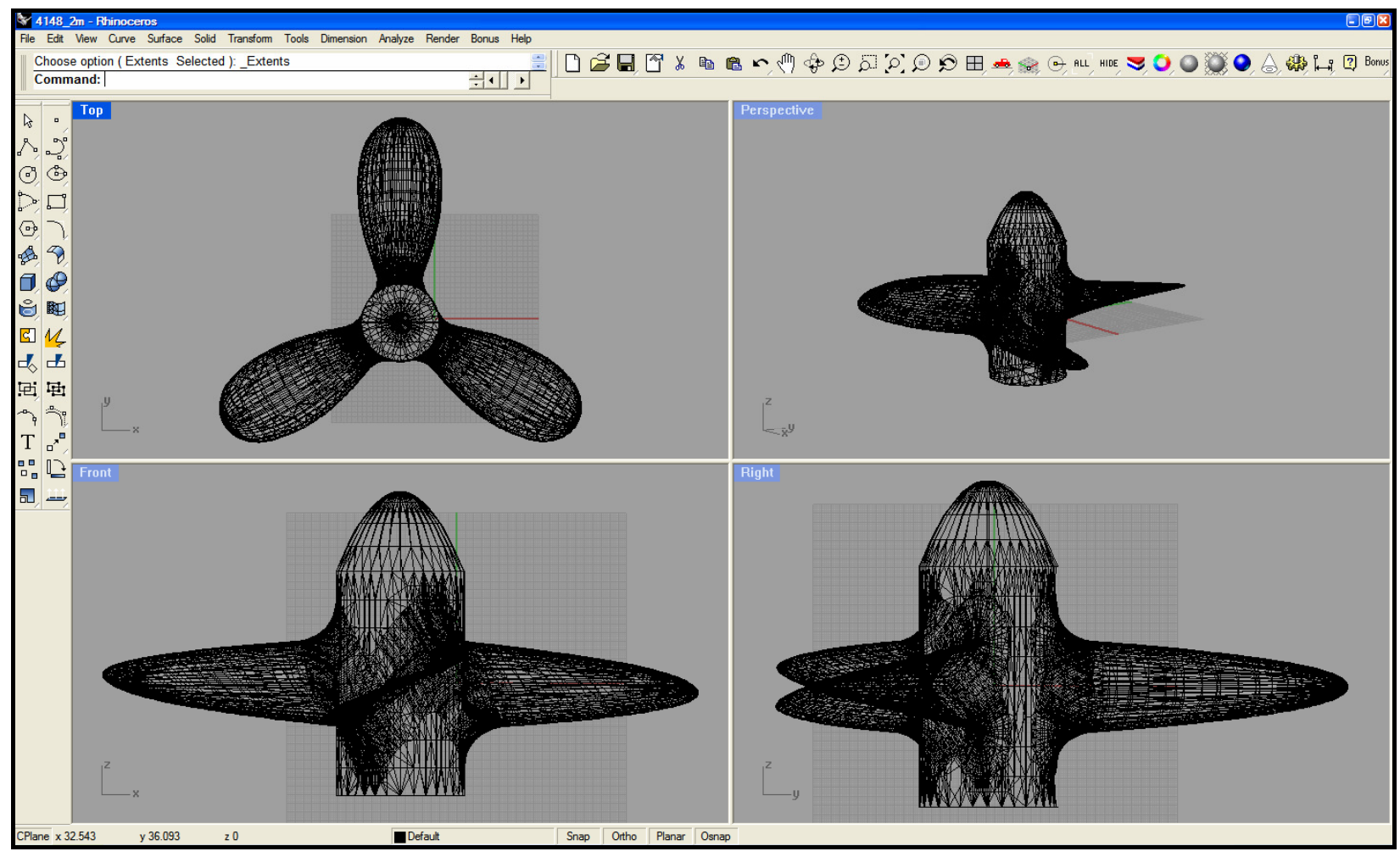

Figure 6-1: Using RHINO ${ }^{\mathrm{TM}}$ to create a .STL file with the 4148-based model propeller.

The printable file made using the 4148-based test case geometry is shown in Figure 6-3.

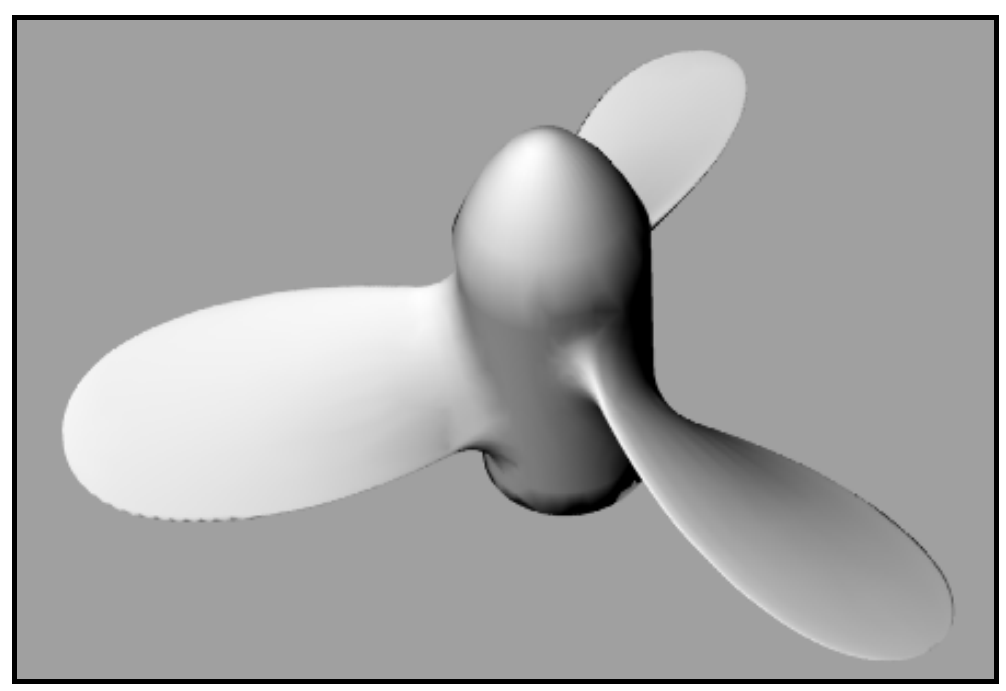

Figure 6-2: Sample propeller .STL file using OpenPVL 4148 geometry. 
This study followed the necessary steps for propeller prototyping as outlined in Fig. 3-1. The OpenPVL files can be saved under a name of the user's choosing. This allows the user to create, compare, and store designs for several different propeller blades. The test case revealed the files which OpenPVL produces for analysis purposes, and showed that the geometry based on the US Navy 4148 propeller could be modeled with these techniques. OpenPVL's versatility allows it to efficiently produce a wide-range of propellers, not limited to AUVs, using this method. 


\section{Conclusions and Future Studies}

\subsection{Conclusions}

OpenPVL has been developed from MPVL [5] to support a propeller design-to-prototype process. MPVL was modified to include 3D printing capabilities, as well as to save the propeller design characteristics into organized files for each test run. Lift coefficient analysis and initial blade parameterization was achieved by using basicPLV. The OpenPVL code was exercised in various parts of this design work, as an interface between the MATLAB ${ }^{\mathrm{TM}}$ propeller geometry outputs and the CAD software, RHINO'M. Additionally, OpenPVL was used to make performance comparisons among different propeller designs. Finally, this study has provided an outline for propeller design and prototyping by describing techniques employed to design in OpenPVL, how create a custom propeller and hub using CAD software, and produce a propeller using an FDM.

An initial design code, basicPVL, allows users to produce a script from which a CAD program can read the propeller design geometry. The PVL-based code generates a series of contour graphs to help the user determine a starting point for the propeller design geometry. However, it is not very accessible to new users, and has limited design inputs. The comparison of an existing design to an optimized design using propeller lifting line code improved existing propellers and contributed to the process of choosing future off-the-shelf propellers.

OpenPVL guides the user through the process of designing an efficient propeller and creating propeller geometry files which interface with a CAD program. The CAD program allows the user to create a 3D-printable file and to add a custom propeller hub, fillets and a fairing, if desired. Investigations into the 3D printing process revealed that ABS-PC is the best choice of printing material in an FDM machine. Casting a mold from a propeller prototype would afford additional freedom of propeller material choices and manufacturing capabilities.

The test case of an off-the-shelf propeller used the Bollard Test and the Moving Cart test to investigate propeller thrust performance. The test results showed that the performance of off- 
the-shelf propellers was limited, in terms of producing vehicle speed and power consumption. This substantiates the need for software capable of custom-designed propeller blades. The experiment would be well-supplemented by experimenting with additional motor gear boxes, to allow for a different range of rotational speeds tested.

Initial design decisions from basicPVL code for the case study of an AUV chose a threebladed $0.625 \mathrm{~m}$ diameter propeller at 2RPS, with a hub located at $r / R(0.2)$ and an efficiency of 0.7091. Final propeller design modified the chord distribution to improve distributed lift coefficients and to ensure that shallow-water operations would not experience cavitation effects. The final design geometry used the same values of the propeller blade number, diameter, RPS and hub location as the initial design, but at an efficiency of 0.7642 . While off-the-shelf propellers, such as those designed for model airplanes, can have high calculated efficiencies relative to actuator disk theory, the lift coefficient at each radial position and the resulting stalling effects must be considered.

OpenPVL demonstrated the ability to perform an analysis for the custom-designing and rapid-prototyping of propellers. Single Propeller Design, an easy-to-use and modify GUI in the OpenProp design suite, uses OpenPVL to create two propeller design files from which propellers may be printed, either as a complete propeller and hub, or as separate blades, for larger propeller designs. The files can be used for direct comparison among multiple designs. In this way, OpenPVL promotes a rapid transition between the propeller design and the optimized prototype.

\subsection{Developmental Goals for OpenPVL}

OpenPVL was initiated by the opportunity to add design features to MPVL [5]. Likewise, additional tools will be added to the OpenProp software suite. Several plans for future development include:

- Propeller motor-matching techniques.

- Propeller hub and fairing designs.

- An iterative optimization process for chordlength, the pitch to diameter ratio, and the camber and thickness ratio. 
- Cavitation number calculation through automated Brockett diagrams.

- Ducted propeller capabilities.

- Ability to interface with CAD programs such as SolidWorks ${ }^{\mathrm{TM}}$, in addition to RHINOTM.

- Finite Element Analysis (FEA)

Most significantly, OpenPVL is readily accessible and modifiable by all propeller users; it provides a basic platform which will easily incorporate the addition of advanced codes and design tools. This propeller design tool is ready for immediate use in streamlined propeller prototyping and also holds many opportunities for future expansion and development. 


\section{References}

[1] M. Purcell, C. von Alt, B. Allen, T. Austin, N. Forrester, et al., "New capabilities of the REMUS autonomous underwater vehicle," OCEANS 2000 MTS/IEEE Conference and Exhibition, vol. 1, pp. 147-151, September, 2000.

[2] "Timetable: Development of the Propeller," 2002. http://www.mh-aerotools.de/airfoils/prophist.htm. Retrieved May 13, 2006.

[3] Carlton, J. S. Marine Propellers and Propulsion. Oxford: Butterworth-Heinemann Ltd., 1994.

[4] J. E. Kerwin. Hydrofoils and Propellers. Cambridge, MIT CopyTech, 2001.

[5] H. Chung, "An Enhanced Propeller Design Program Based on Propeller Vortex Lattice Lifting Line Theory," Masters Thesis, Massachusetts Institute of Technology, June 2007.

[6] Coney, William B., Ching-Yeh Hsin, and Justin E. Kerwin. MIT-PLL-2 Report and User's Manual. Cambridge,MA: Massachusetts Institute of Technology, 1986.

[7] R. Kimball, "Huang Body-1 MTWAKE screen matching experimental wake MTSOL grid finer near body," personal communication, April 19, 2006.

[8] Abbott, Ira H., and Albert E. Von Doenhoff. Theory of Wing Sections. New York: Dover Publications, 1959.

[9] T. Brockett, "Minimum Pressure Envelopes for Modified NACA-66 Sections with NACA a-0.8 Camber and BuShips Type I and Type II Sections," DTMB Report 1780, February 1966.

[10] J. N. Newman, Marine Hydrodynamics. Cambridge, MA: The MIT Press, 1977, pp. 206208.

[11] "Titan FDM Real Materials." http://www.StrataSys TM.com. Accessed 05JUL2007.

[12] "Machinist-Materials, Plastics Comparison Table." http://www.machinist-materials.com/comparison table for plastics.htm. Accessed 03JUL2007.

[13] R. Camilli, "Cosmos FloWorks ${ }^{\mathrm{TM}}$ AUV Modeling," personal communication, April 21, 2006.

[14] C. Roman, "SeaBED MATLAB ${ }^{\text {TM }}$ Code," personal communication, April 3, 2006.

[15] K. P. D'Epagnier, "AUV Propellers: Optimal Design and Improving Existing Propellers for Greater Efficiency," in Proc. OCEANS, Boston, MA, 2006.

[16] Dr. S. A. Kinnas, "University/Navy/Industry Consortium on Cavitation of High Speed Propulsors," fifth meeting, June $1^{\text {st }}$ and $2^{\text {nd }}, 1995$. 
Appendix A: OpenPVL MATLAB ${ }^{\mathrm{TM}}$ Source Code 


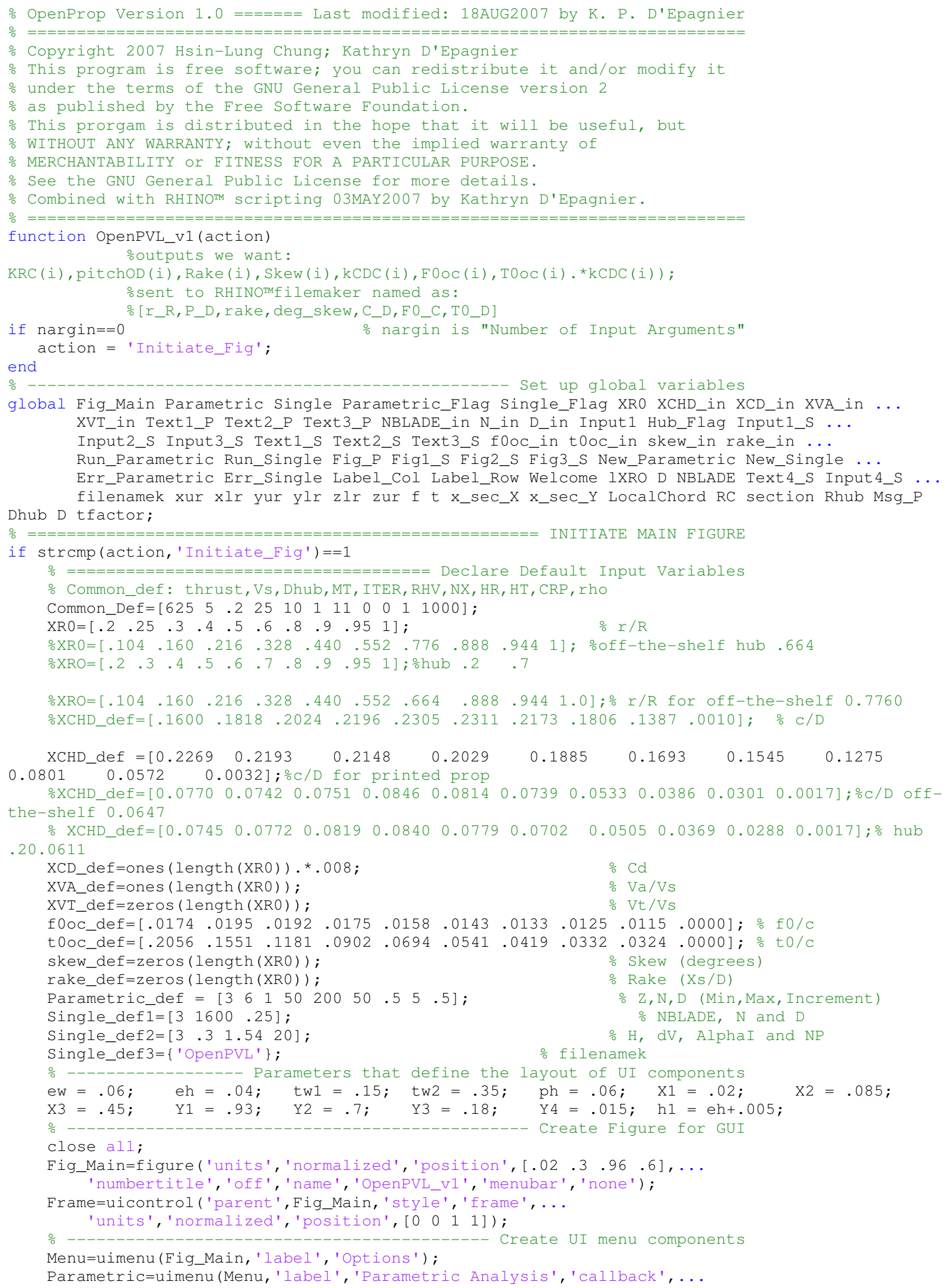




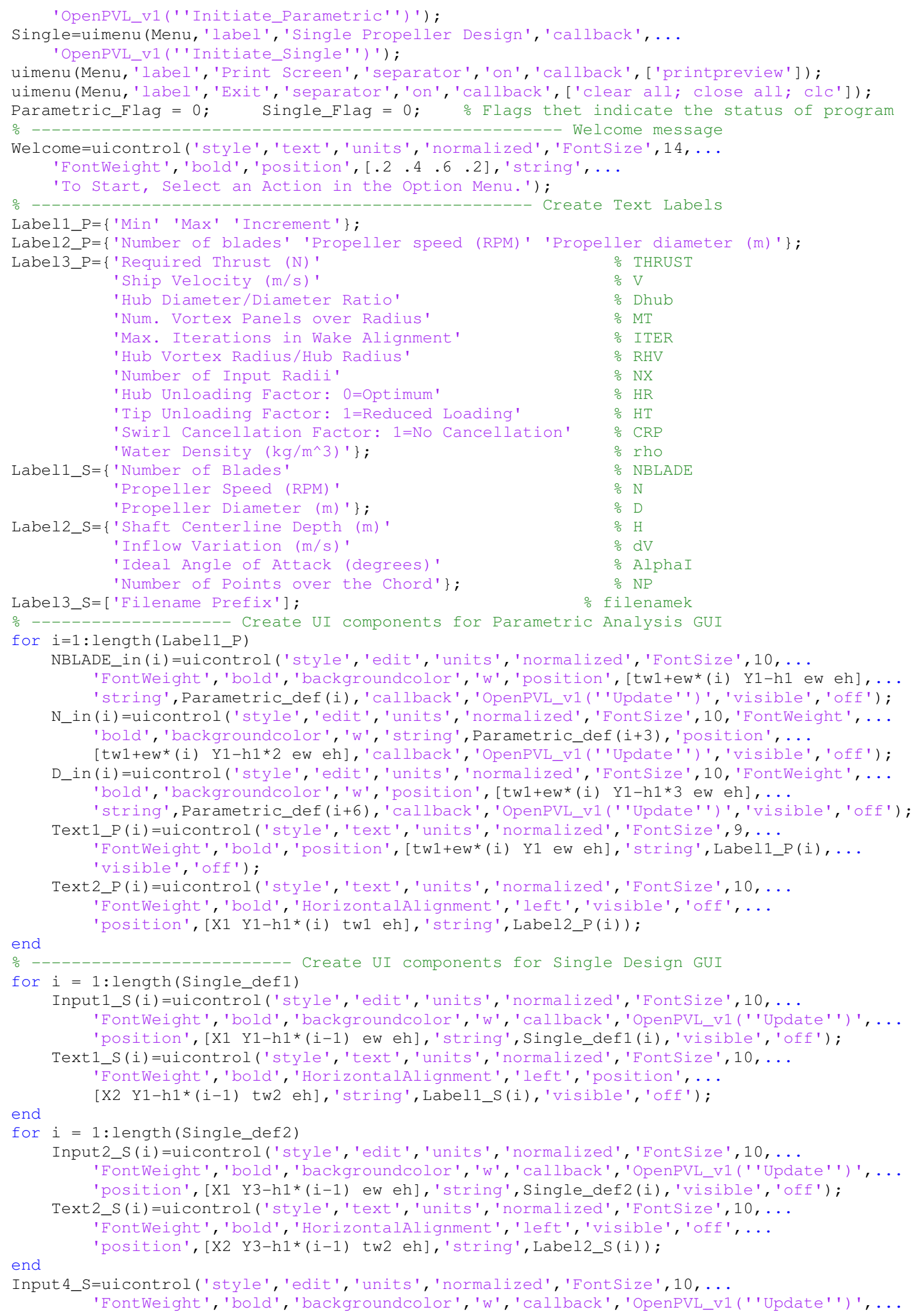


'position', [X3 Y3-eh 3*ew eh], 'string', Single_def3,' 'visible', 'off');

Text4_s=uicontrol ('style', 'text', 'units', 'normalized', 'Fontsize', 10, ...

'FontWeight' ' 'bold', 'HorizontalAlignment ', 'left' ' 'visible', 'off',...

'position', [X3 Y3 tw2 eh], 'string',Label3_S);

\% - - - - - - ------------------ Create UI components for Common Inputs

for $i=1:$ length (Common_Def)

Input1 (i)=uicontrol('style', 'edit', 'units', 'normalized', 'Fontsize',10,...

'FontWeight', 'bold', 'backgroundcolor' ' 'w', 'callback' , 'OpenPVL_v1 (' 'Update' ' ) ', ...

'position', [X1 Y2-h1*(i-1) ew eh],'string',Common_Def(i), 'visible','off');

Text3_P(i)=uicontrol ('style', 'text', 'units', 'normalized', 'Fontsize', 10,...

'FontWeight', 'bold', 'HorizontalAlignment' , 'left' , 'visible', 'off' ,...

'position', [X2 Y2-h1*(i-1) tw2 eh], 'string', Label3_P(i));

end

Hub_Flag=uicontrol ('style', 'checkbox', 'units', 'normalized', 'Fontsize',10,...

'FontWeight','bold', 'position', [X3 Y1 tw2 eh],'value',1,'callback',...

'OpenPVL_v1(''Update'')', 'string', 'Hub Image Flag (Check for YES)', 'visible', 'off');

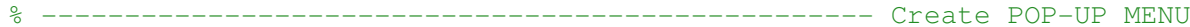

Input3_s (1)=uicontrol ('style', 'popupmenu', 'units', 'normalized', 'Fontsize',8,...

'FontWeight', 'bold', 'backgroundcolor', 'w', 'callback', 'OpenPvL_v1 (' 'Update' ') ', 'position', ...

[X3 Y1-eh*3 tw1 eh], 'string', \{'NACA a=0.8' 'Parabolic'\},'visible', 'off');

Text3_S(1)=uicontrol ('style', 'text', 'units', 'normalized', 'Fontsize',10,...

'FontWeight', 'bold', 'HorizontalAlignment','left','position', [X3 Y1-eh*2 tw1 eh],...

'string', 'Meanline Type: ', 'visible', 'off');

Input3_S(2)=uicontrol ('style', 'popupmenu', 'units', 'normalized', 'FontSize',8,...

'FontWeight', 'bold', 'backgroundcolor', 'w' , 'callback', 'OpenpvL_v1 (' 'Update' ') ', 'position' , .. .

[X3+tw1+ew Y1-eh*3 tw1 eh], 'string', \{'NACA 65A010' 'Elliptical' 'Parabolic' 'NACA

Thickened for 3-D Printing'\},...

'visible', 'off');

Text3_s (2)=uicontrol ('style', 'text', 'units', 'normalized', 'Fontsize', 10, 'FontWeight',...

'bold', 'HorizontalAlignment', 'left', 'position', [X3+tw1+ew Y1-eh*2 tw1 eh],...

'string', 'Thickness Form: ', 'visible', 'off');

\% - - - - - - - create UI components for inputs on the right of the GUI

for $j=1$ :length $(\mathrm{XRO})$

Label_Row (j)=uicontrol ('style', 'edit', 'units', 'normalized', 'Fontsize', 10,...

'FontWeight', 'bold', 'position', [X3 Y2-h1*j ew eh], 'string', XRO(j), ...

'visible', 'off', 'enable', 'inactive');

XCHD_in $(j)=u i c o n t r o l(' s t y l e '$ ', 'edit', 'units', 'normalized', 'Fontsize', $10, \ldots$ 'FontWeight', 'bold', 'backgroundcolor', 'w', 'string', XCHD_def(j), 'position',... [X3+ew Y2-h1*j ew eh], 'callback', 'OpenPVL_v1(''Update' ')', 'visible', 'off');

XCD_in $(j)=$ uicontrol ('style', 'edit', 'units', 'normalized', 'Fontsize', 10, . . 'FontWeight' , 'bold', 'backgroundcolor' ' 'w', 'string', XCD_def(j), 'position', ... [X3+ew*2 Y2-h1*jew eh], 'callback', 'OpenPVL_v1(''Update' ') ', 'visible', 'off') ;

XVA_in $(j)=u i c o n t r o l(' s t y l e '$, 'edit', 'units', 'normalized', 'Fontsize', 10, ... 'FontWeight' , 'bold', 'backgroundcolor' ' 'w', 'string', XVA_def(j), 'position', ... [X3+ew*3 Y2-h1*jew eh], 'callback', 'OpenPVL_v1(''Update'' ') ', 'visible', 'off');

XVT_in (j)=uicontrol('style', 'edit', 'units', 'normalized', 'FontSize', 10, ... 'FontWeight', 'bold', 'backgroundcolor' ' 'w', 'string', XVT_def (j), 'position', ... [X3+ew*4 Y2-h1*j ew eh], 'callback', 'OpenPVL_v1(''Update'')',' 'visible', 'off');

f0oc_in $(j)=$ uicontrol ('style', 'edit', 'units', 'normalized', 'Fontsize', 10, .. 'FontWeight', 'bold', 'backgroundcolor', 'w', 'string',f0oc_def(j), 'position',... [X3+ew*5 Y2-h1*j ew eh], 'callback', 'OpenPVL_v1 (''Update'') ', 'visible', 'off');

t0oc_in $(j)=$ uicontrol ('style', 'edit', 'units', 'normalized', 'Fontsize', 10,... 'FontWeight', 'bold', 'backgroundcolor', 'w', 'string', t0oc_def(j), 'position',... [X3+ew*6 Y2-h1*j ew eh], 'callback', 'OpenPVL_v1 (''Update' ') ', 'visible', 'off');

skew_in $(j)=u i c o n t r o l(' s t y l e '$, 'edit', 'units', 'normalized', 'FontSize', 10, ... 'FontWeight', 'bold', 'backgroundcolor', 'w', 'string', skew_def(j), 'position',... $[\mathrm{X} 3+\mathrm{ew}$ 7 Y2-h1*jew eh], 'callback', 'OpenPVL_v1(''Update'') ', 'visible', 'off') ;

rake_in $(j)=u i c o n t r o l(' s t y l e '$, 'edit', 'units', 'normalized', 'Fontsize', 10,... 'FontWeight', 'bold', 'backgroundcolor', 'w', 'string', rake_def(j), 'position',... [X3+ew*8 Y2-h1*j ew eh], 'callback', 'OpenPVL_v1 (''Update'') ', 'visible', 'off');

Msg_P $(j)=u i c o n t r o l(' s t y l e '$, 'edit', 'units', 'normalized', 'Fontsize', 10, ... 'FontWeight', 'bold', 'backgroundcolor', 'w', 'string', rake_def(j), 'position', ... end $[\mathrm{X} 3+\mathrm{ew}$ 9 Y2-h1*jew eh], 'callback', 'OpenPVL_v1(''Update'')',' 'visible', 'off');

ColName $=\{' r / R$ ' 'c/D' 'Cd' 'Va/Vs' 'Vt/Vs' 'fo/c' 'to/c' 'Skew' 'Xs/D'\};

for $i=1$ :length (ColName)

Label_Col(i)=uicontrol ('style', 'edit', 'units', 'normalized', 'Fontsize', 10,... 


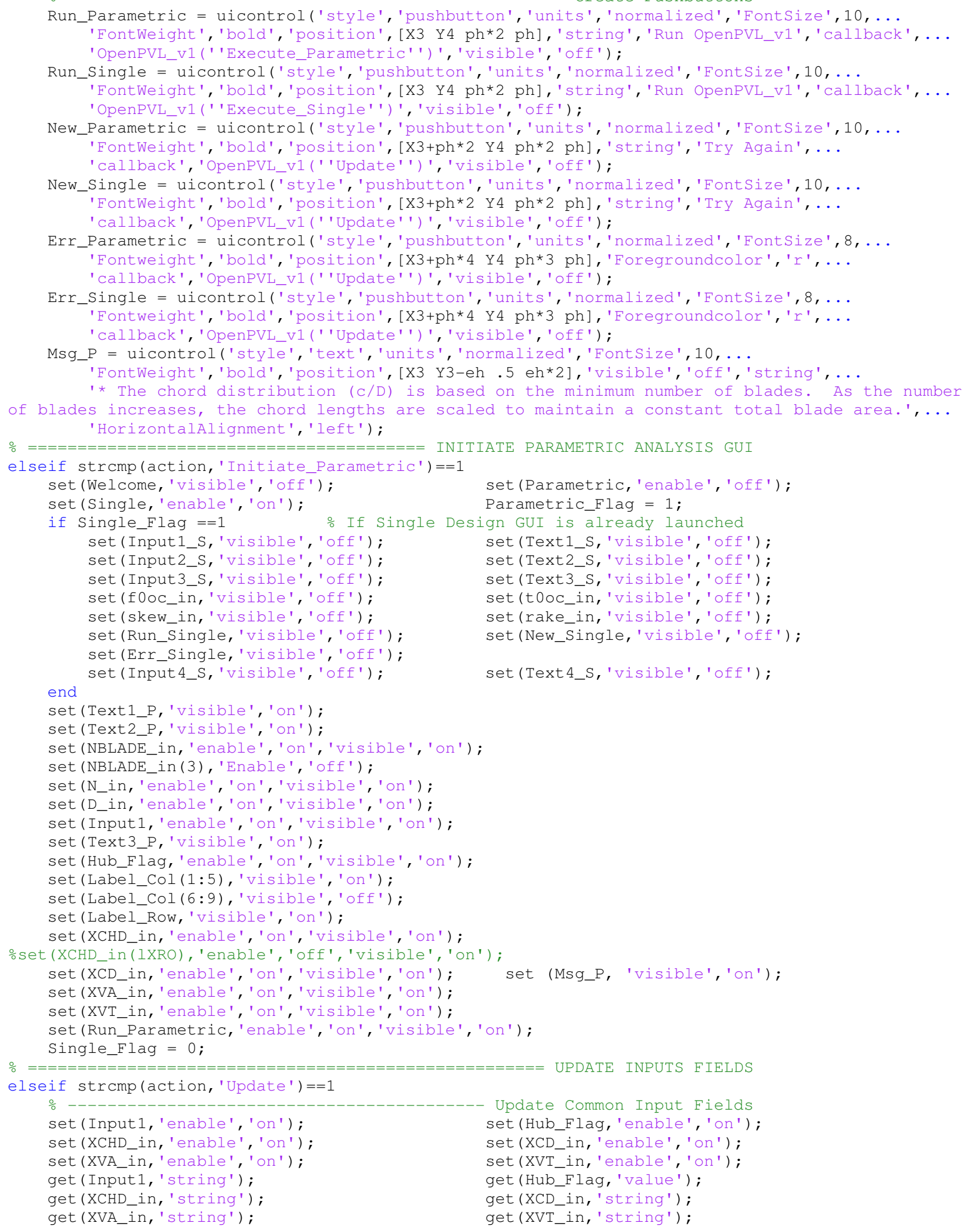




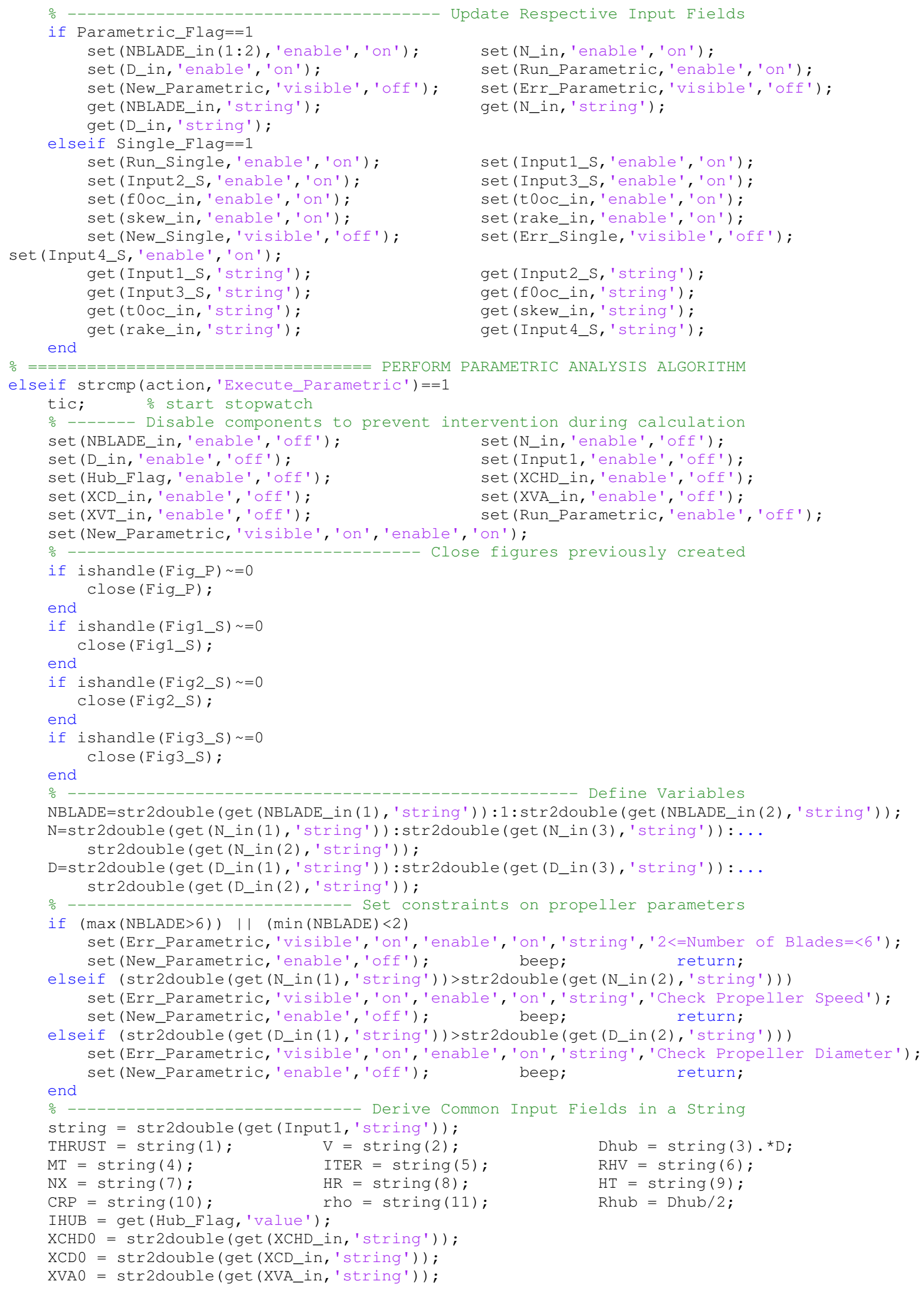




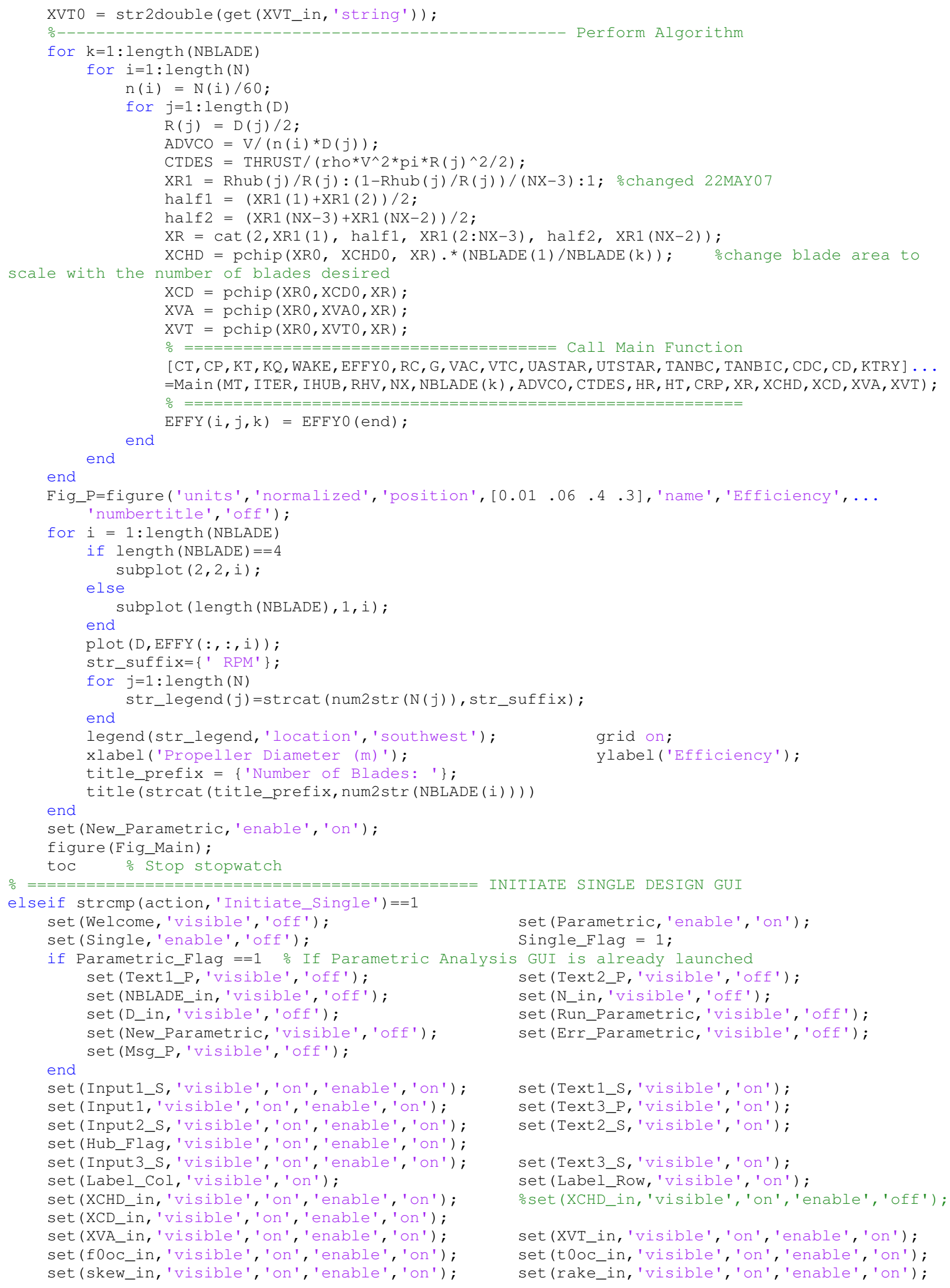




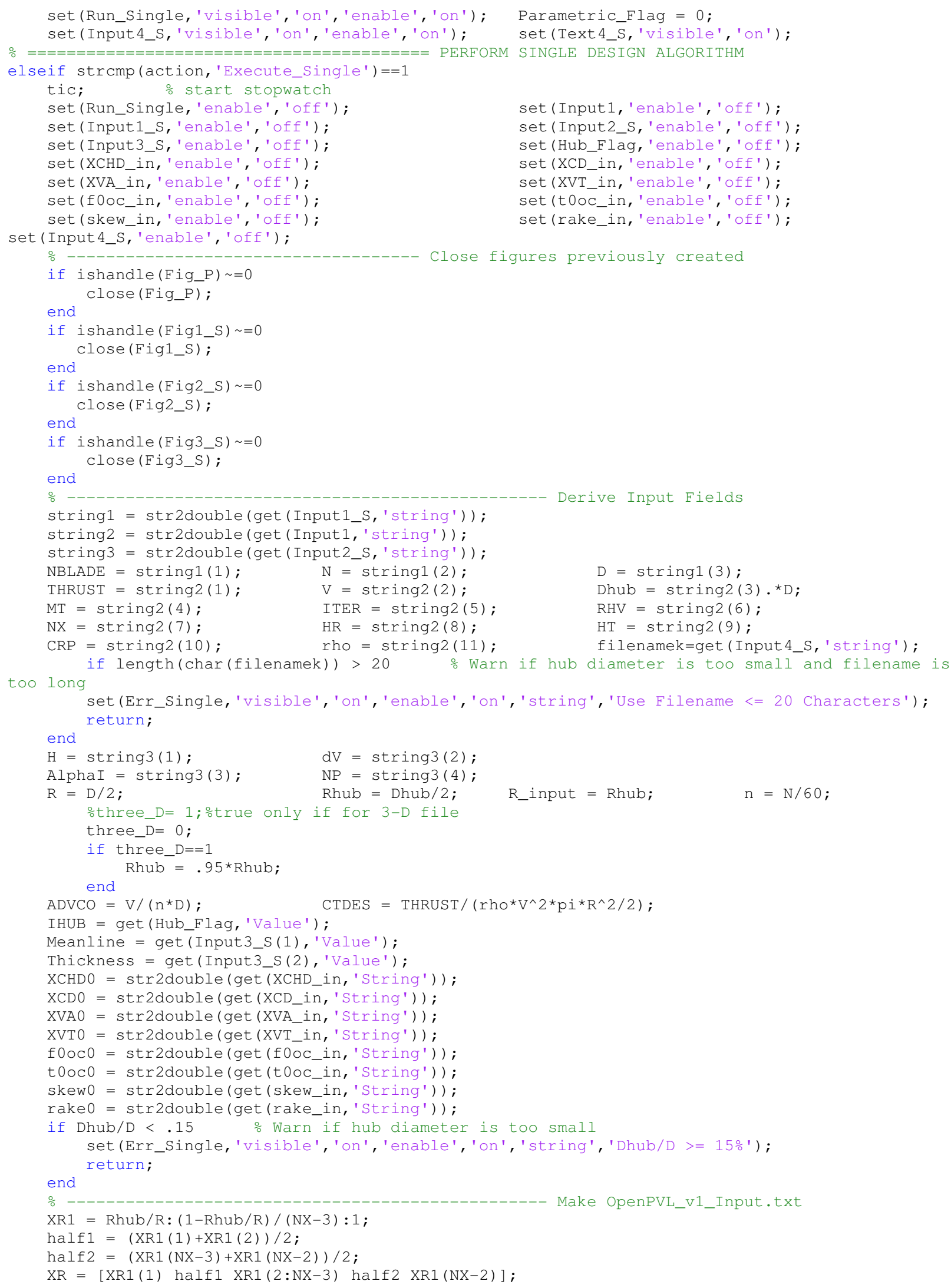


$\mathrm{XCHD}=\operatorname{pchip}(\mathrm{XRO}, \mathrm{XCHD} 0, \mathrm{XR}) ;$

$\mathrm{XCD}=\operatorname{pchip}(\mathrm{XRO}, \mathrm{XCD} 0, \mathrm{XR}) ;$

$\mathrm{XVA}=\operatorname{pchip}(\mathrm{XRO}, \mathrm{XVA} 0, \mathrm{XR}) ;$

$\mathrm{XVT}=\operatorname{pchip}(\mathrm{XRO}, \mathrm{XVTO}, \mathrm{XR})$;

Flag1 = datestr $($ now, 31);

prop_name = char (filenamek); filename

fid = fopen ([prop_name, '_Input.txt'], 'w');

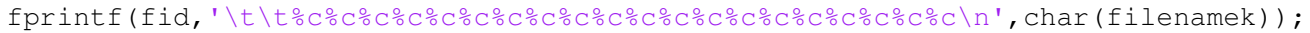

fprintf(fid,'_Input.txt for Open_PVL_v1 \n',Flag1);

fprintf(fid, '\n\%.0f \t\tNumber of Vortex Panels over the Radius $\backslash n$ ', MT);

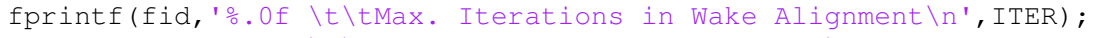

fprintf(fid,' $\%$.Of $\backslash t \backslash$ tHub Image Flag: $1=$ YES, $0=$ NO \n', IHUB);

fprintf(fid,' '\%.1f \tHub Vortex Radius/Hub Radius\n', RHV);

fprintf(fid,' $\%$.of $\backslash t \backslash t$ Number of Input Radii\n',NX);

fprintf(fid, '\%.0f $\backslash t \backslash t$ Number of Blades $\backslash n$ ', NBLADE);

fprintf(fid,' $\%$. $f$ \tAdvance Coef., J, Based on Ship Speed $\backslash n$ ', ADVCO);

fprintf(fid,' $\%$.3f \tDesired Thrust Coef., Ct\n',CTDES);

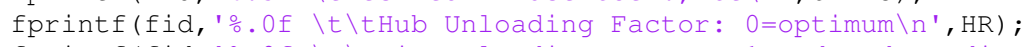

fprintf(fid,' $\%$. of \t ttTip Unloading Factor: 1 =Reduced Loading $\backslash n$ ',HT);

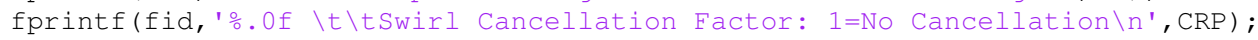

fprintf(fid,'r/R \t $\mathrm{C} / \mathrm{D} \quad \backslash \mathrm{t} \quad \mathrm{Cd} \backslash \mathrm{t} \quad \mathrm{Va} / \mathrm{Vs} \quad \mathrm{Vt} / \mathrm{Vs} \backslash \mathrm{n}$ ');

for $i=1:$ NX end

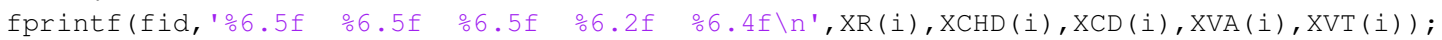

fprintf(fid, ' $\backslash \mathrm{nr} / \mathrm{R}$ is the ratio of the radial location, r, to the total length of the blade radius, $\mathrm{R} .\left(\mathrm{n}^{\prime}\right)$;

fprintf(fid,'C/D is the ratio of chordlength to diameter. $\left.\backslash \mathrm{n}^{\prime}\right)$;

fprintf(fid,'Cd is the drag coefficient. \n');

fprintf(fid,'Va/Vs is the axial inflow velocity ratio (is 1, assuming uniform inflow in open water). $\left.\backslash \mathrm{n}^{\prime}\right)$;

fprintf(fid,'Vt/Vs is the tangential inflow velocity ratio (is 0 , assuming uniform inflow in open water). (n');

fclose (fid);

$\therefore=0==============================================$ Call Main Function

[CT, CP , KT, KQ, WAKE, EFFY, RC, G, VAC, VTC, UASTAR, UTSTAR, TANBC, TANBIC, CDC, CD, KTRY, KRC] ... =Main (MT, ITER, IHUB, RHV, NX, NBLADE, ADVCO, CTDES, HR, HT, CRP, XR, XCHD, XCD, XVA, XVT, filenamek) ;

음 -

Fig1_S = figure('units', 'normalized', 'position', [.01 .06 .4 .3], 'name',... 'Graphical Report', 'numbertitle', 'off');

subplot $(2,2,1)$;

plot $(\mathrm{RC}, \mathrm{G})$;

xlabel('r/R'); $\quad$ ylabel('Non-Dimensional Circulation'); grid on;

Titlestring=strcat (' J=', num2str (ADVCO, '\%10.3f'), ' ; Ct=', num2str (CT (KTRY), '⒑3f'), ...

'; Kt=', num2str(KT (KTRY), '\%10.3f'), ' ; Kq=', num2str (KQ (KTRY), '⿳10.3f'), ...

'; leta=', num2str(EFFY(KTRY), '⒑3f')) ;

title(Titlestring);

subplot $(2,2,2)$;

plot (RC, VAC, '-b ', RC, VTC, '--b', RC, UASTAR, '-.r', RC, UTSTAR, ' : r') ;

xlabel('r/R'); legend('Va/Vs', 'Vt/Vs', 'Ua*/Vs', 'Ut*/Vs'); grid on;

subplot $(2,2,3)$;

plot (RC, TANBC, '--b', RC, TANBIC, '-r') ;

xlabel('r/R'); $\quad$ ylabel('Degrees'); $\quad$ grid on; legend('beta', 'betaI');

subplot $(2,2,4)$;

plot $(\mathrm{RC}, \mathrm{CDC})$;

xlabel('r/R'); $\quad$ ylabel('C/D'); $\quad$ grid on;

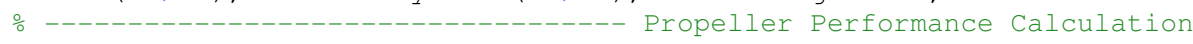

$\mathrm{w}=2{ }^{*} \mathrm{pi}{ }^{*} \mathrm{n} ; \quad \therefore$ Angular velocity $\mathrm{w}$

for $\mathrm{k}=1: \mathrm{MT}$

$\operatorname{Vstar}(k)=\operatorname{sqrt}\left((\operatorname{VAC}(k)+\operatorname{UASTAR}(k))^{\wedge} 2+\left(\mathrm{w}^{*} \mathrm{R} * \mathrm{RC}(\mathrm{k})+\operatorname{VTC}(\mathrm{k})+\operatorname{UTSTAR}(\mathrm{k})\right)^{\wedge} 2\right) i$

$\operatorname{Gamma}(k)=G(k) * 2 * p i * R * V$;

$\mathrm{Cl}(\mathrm{k})=2{ }^{*} \operatorname{Gamma}(\mathrm{k}) /\left(\operatorname{Vstar}(\mathrm{k}){ }^{*} \mathrm{CDC}(\mathrm{k}){ }^{*} \mathrm{D}\right)$;

$\operatorname{dBetai}(k)=\operatorname{atand}\left(\left(\operatorname{tand}(\operatorname{TANBIC}(k)) * \mathrm{w}^{*} \mathrm{RC}(\mathrm{k}) * \mathrm{R}+\mathrm{dV}\right) /(\mathrm{w} * \mathrm{RC}(\mathrm{k}) * \mathrm{R})\right) \ldots$

-atand $\left(\left(\right.\right.$ tand $\left.\left.(\operatorname{TANBIC}(\mathrm{k})){ }^{*} \mathrm{w} * \mathrm{RC}(\mathrm{k}) * \mathrm{R}-\mathrm{dV}\right) /(\mathrm{w} * \mathrm{RC}(\mathrm{k}) * \mathrm{R})\right)$;

end

Sigma $(k)=(101000+r h o * 9.81 *(H-R C(k) * R)-2500) /(r h o * \operatorname{Vstar}(k) \wedge 2 / 2)$; Cavitation Number

$\mathrm{KCl}=[\mathrm{Cl} \mathrm{Cl}(\mathrm{k})]$;

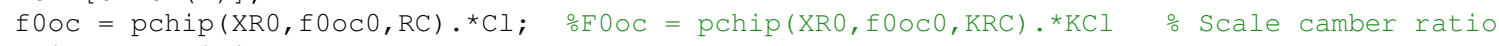

with lift coefficient

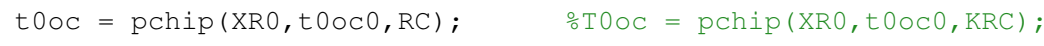




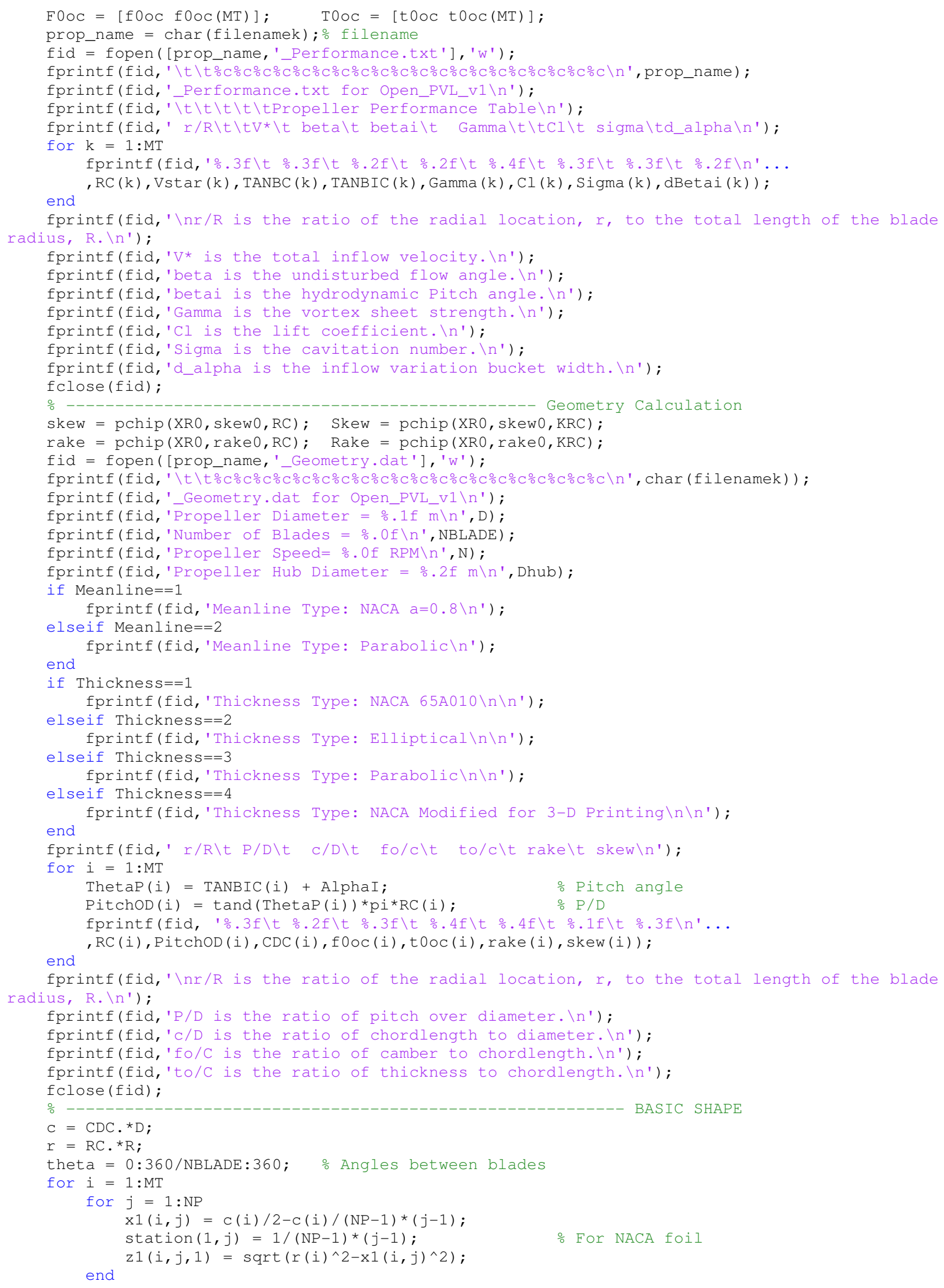




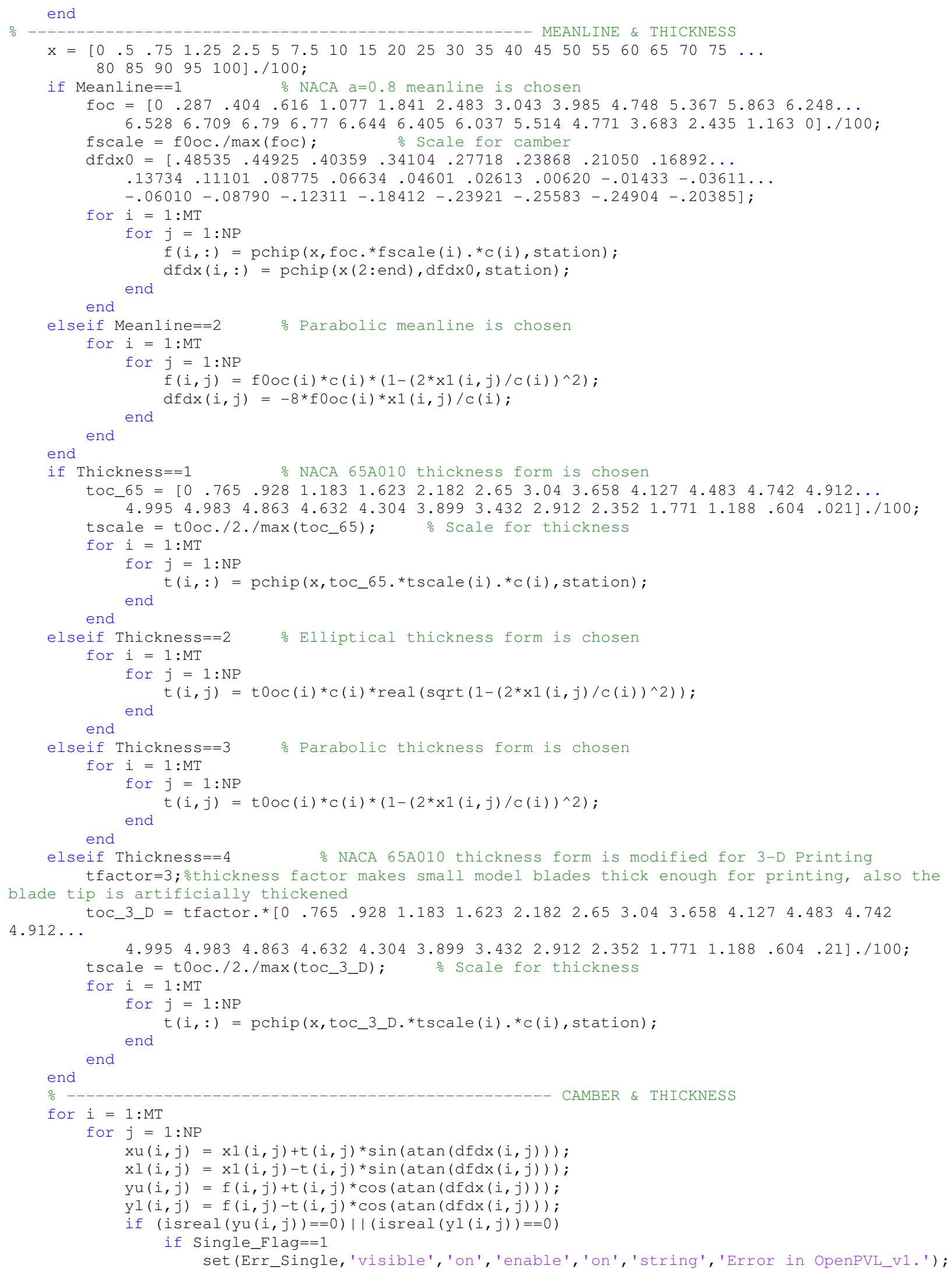




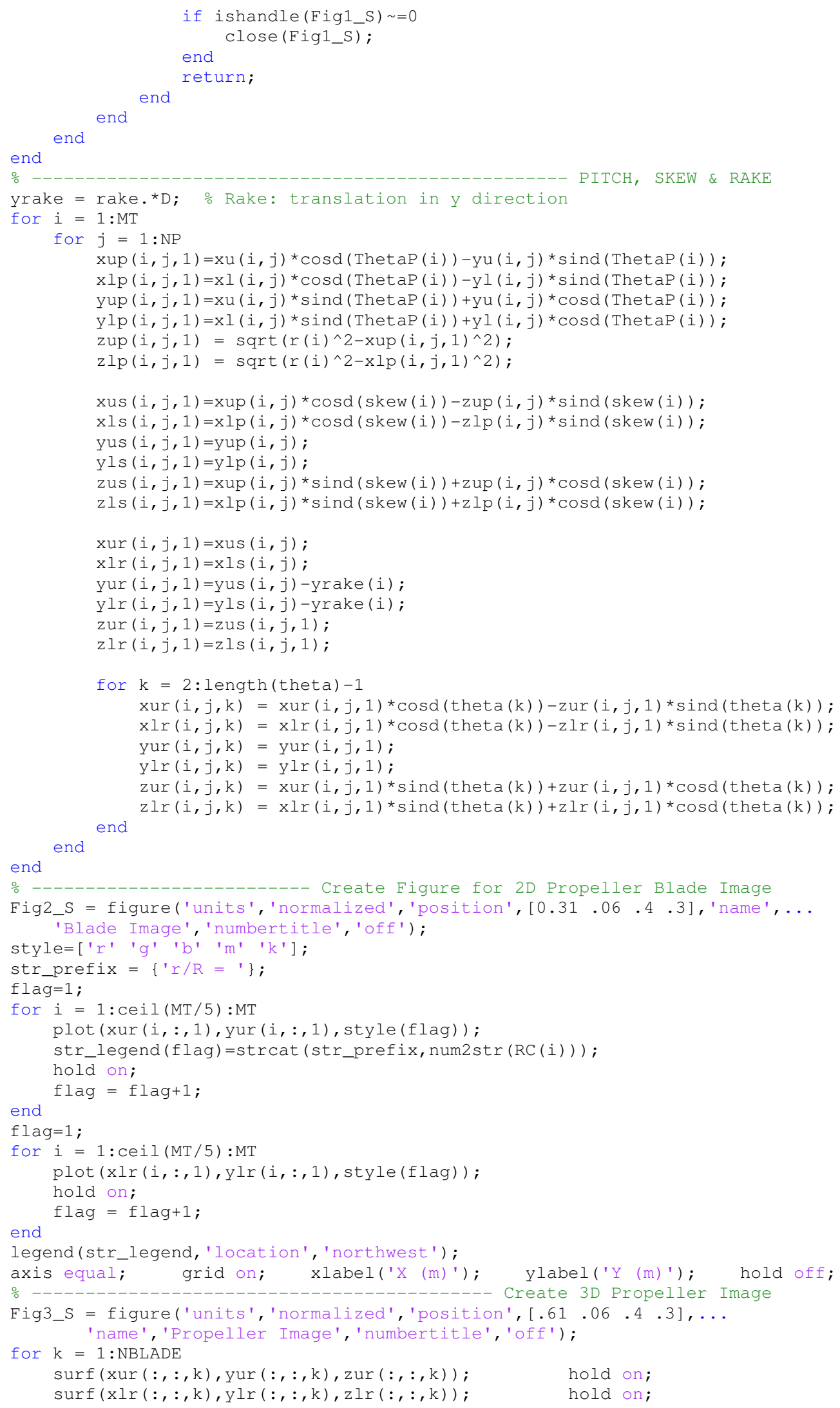




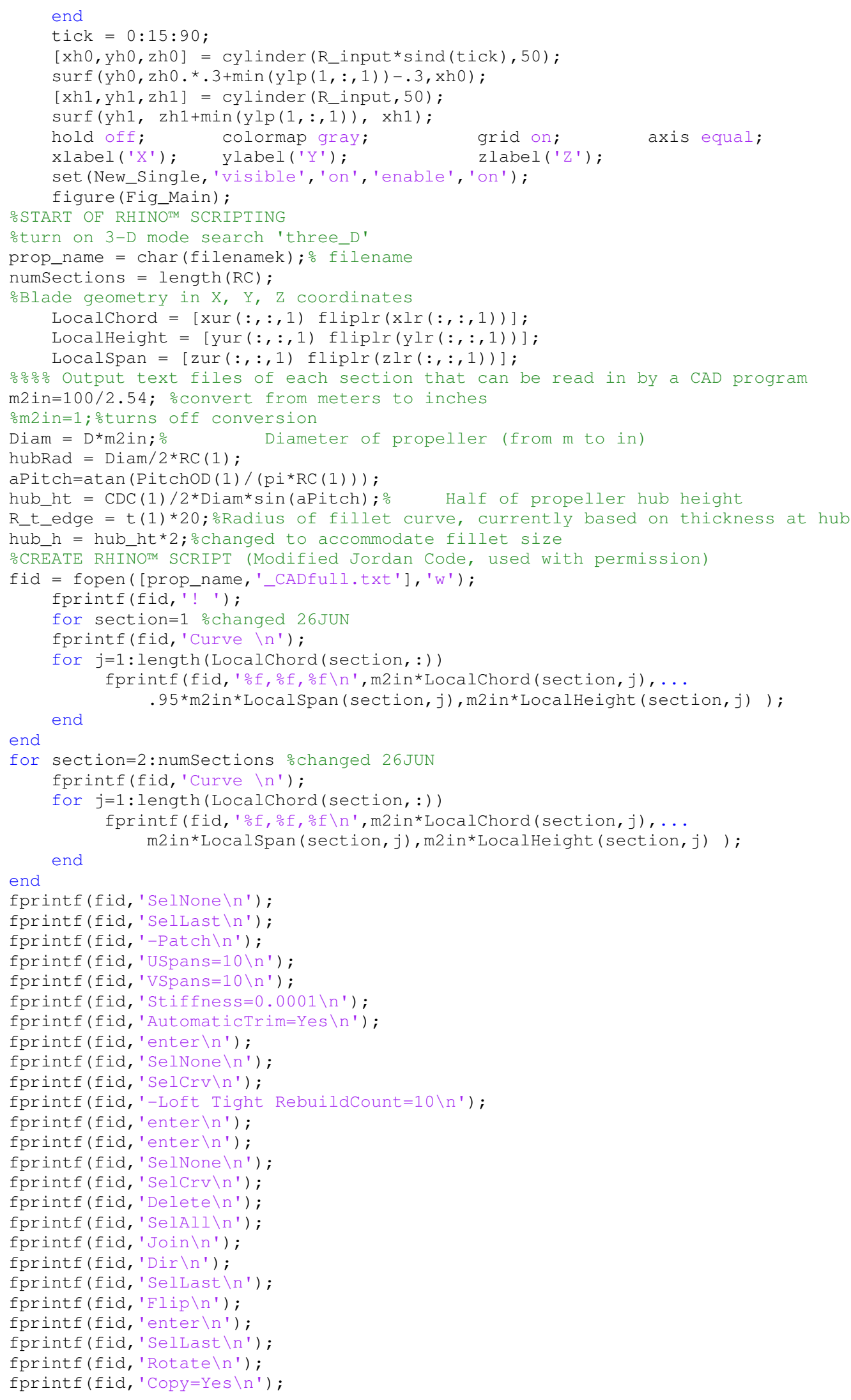




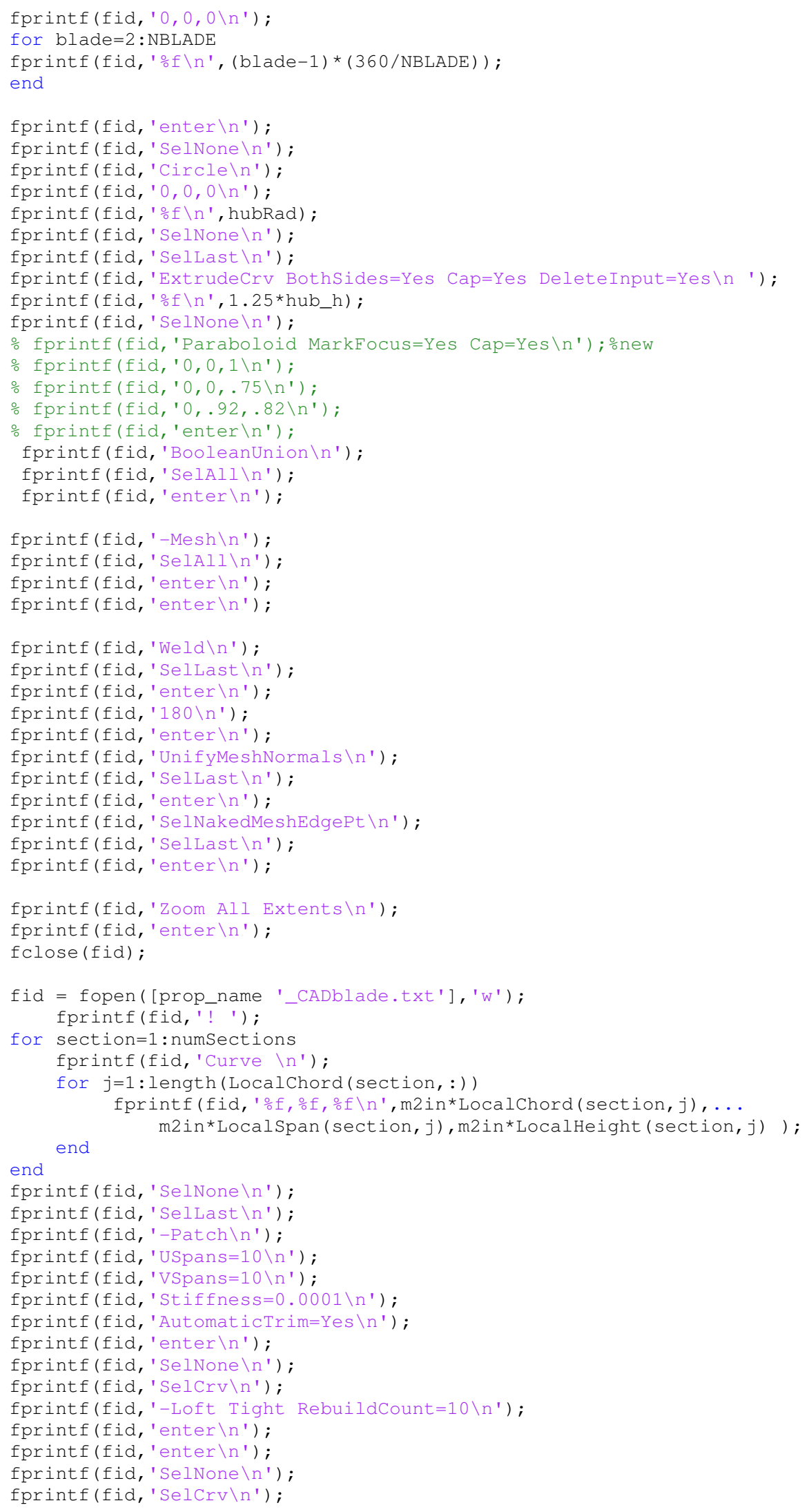




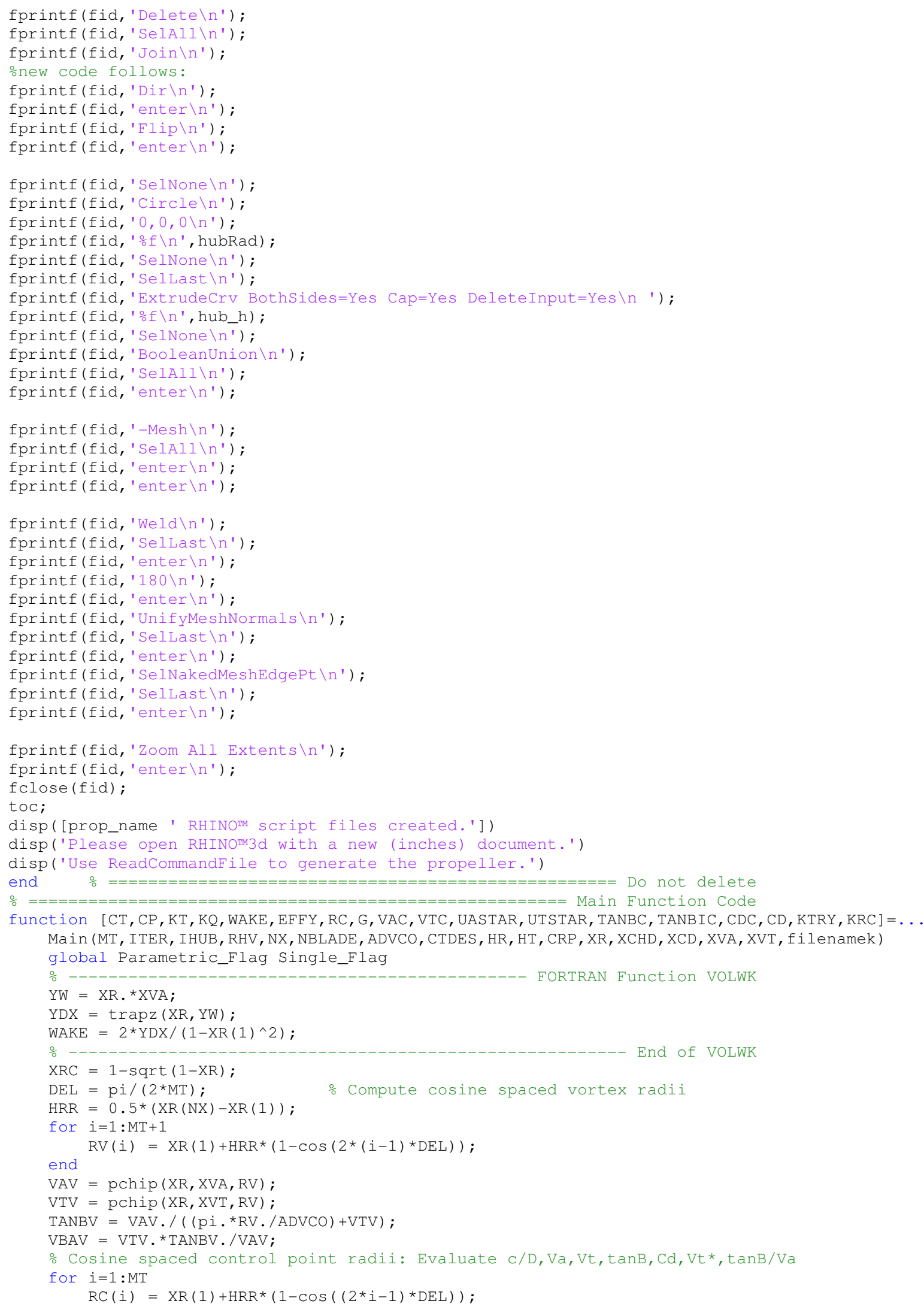




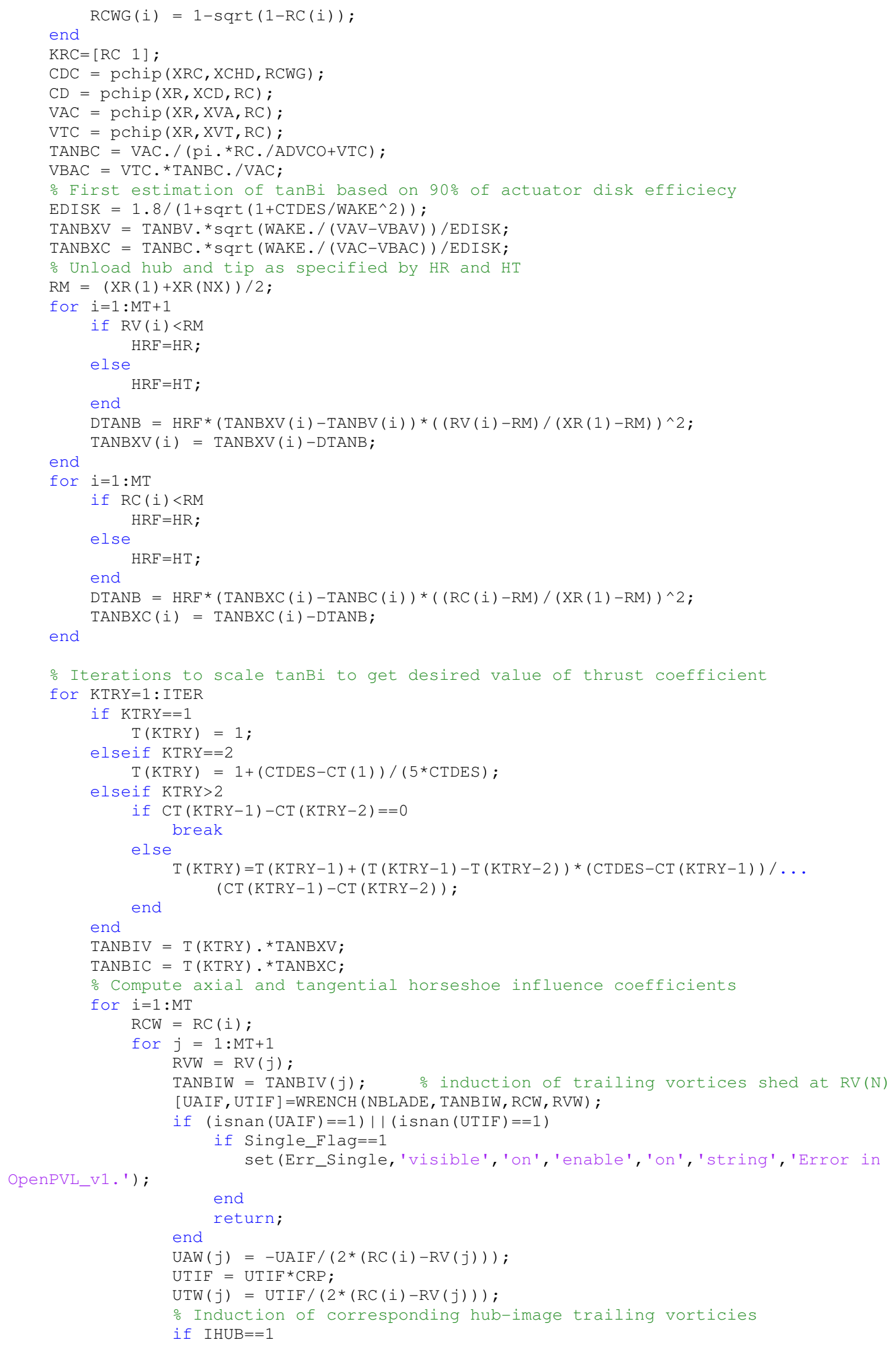




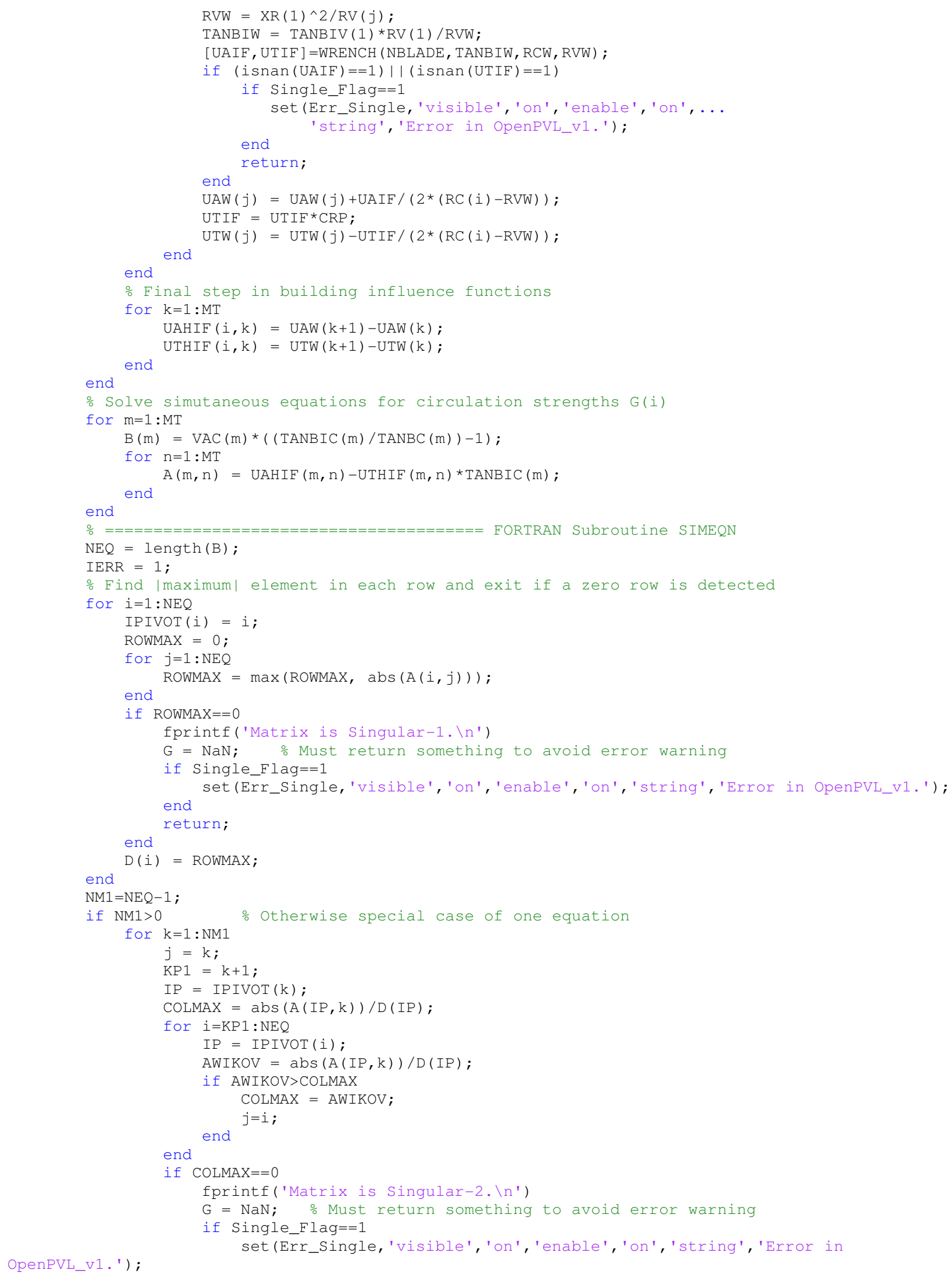




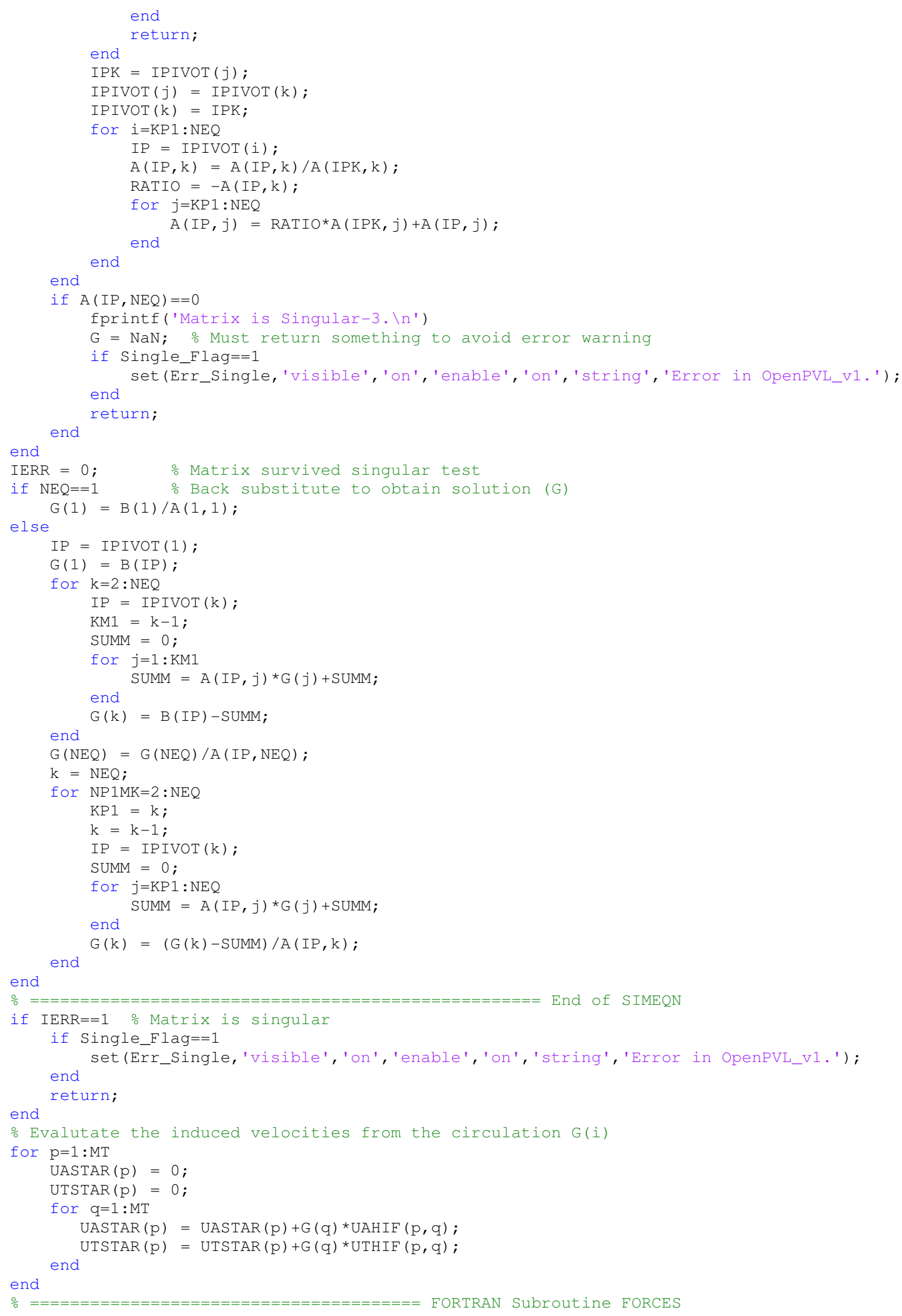




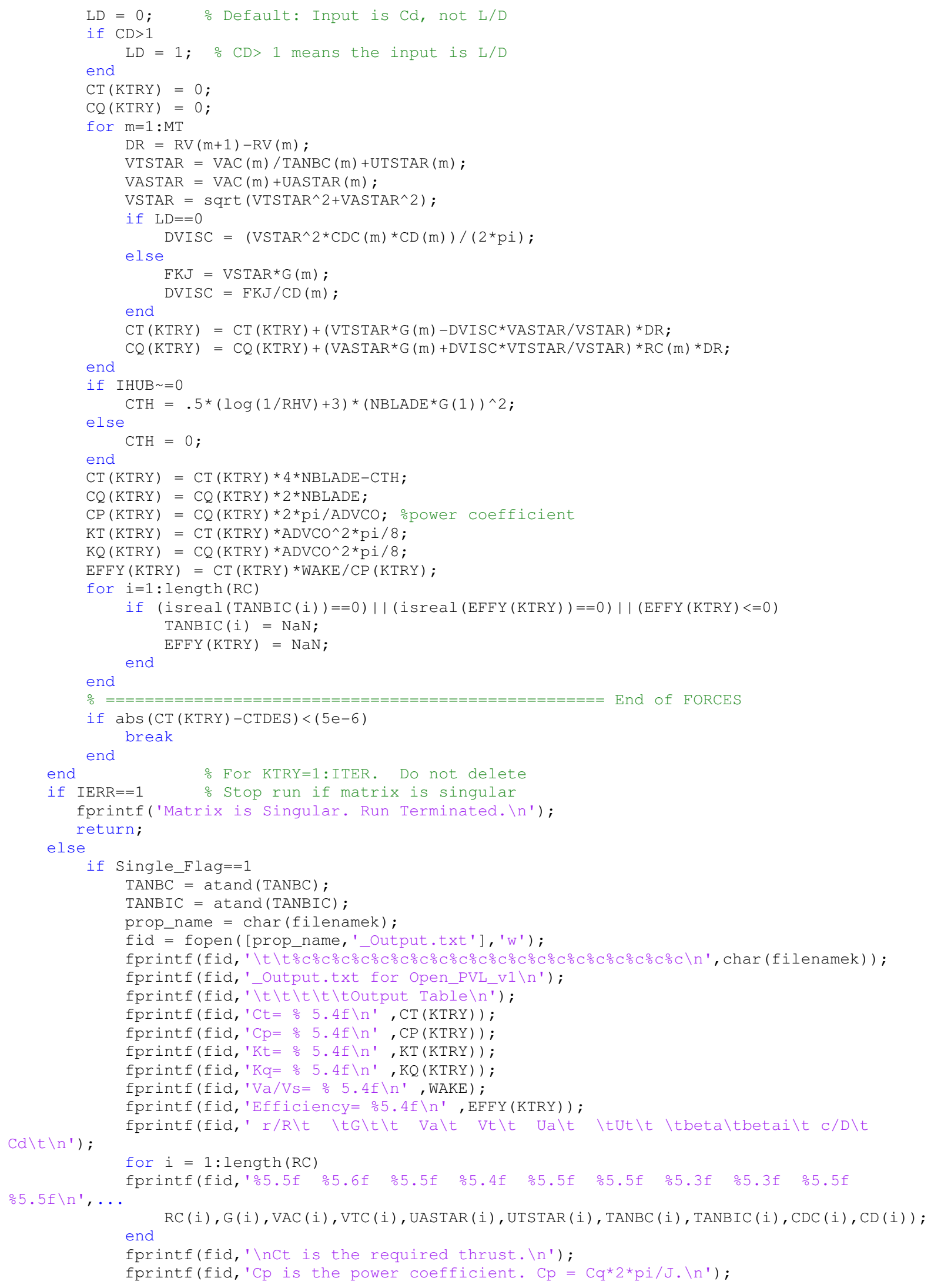


fprintf(fid, 'Kt is the thrust coefficient. Kt $=\mathrm{Ct}^{\star} \mathrm{J}^{\wedge} 2^{\star} \mathrm{pi} / 8$, where $\mathrm{J}$ is the advance coefficient. (n');

fprintf(fid,' Kq is the torque coefficient. $\mathrm{Kq}=\mathrm{Cq}^{*} \mathrm{~J}^{\wedge} 2{ }^{*} \mathrm{pi} / 8 . \backslash \mathrm{n}$ ') ;

fprintf(fid, 'Va/Vs is the axial inflow velocity ratio (is 1, assuming uniform inflow in open water). (n' );

fprintf(fid, 'Efficiency is the ratio of required thrust times the wake to the power coefficient. (n');

fprintf(fid,'r/R is the ratio of the radial location, r, to the total length of the

blade radius, R. \n');

fprintf(fid, 'G is the non-dimensional circulation. In');

fprintf(fid,'Va is the axial inflow velocity. \n');

fprintf(fid, 'Vt is the tangential inflow velocity. \n');

fprintf(fid, 'Ua us the induced axial velocity. \n');

fprintf(fid, 'ut is the induced tangential velocity. $\backslash \mathrm{n}^{\prime}$ );

fprintf(fid, 'beta is the flow angle. \n');

fprintf(fid,' betaI is the hydrodynamic Pitch angle. In');

fprintf(fid,'c/D is the ratio of chordlength to diameter. \n');

fprintf(fid, 'Cd is the drag coefficient. In');

end

end fclose (fid);

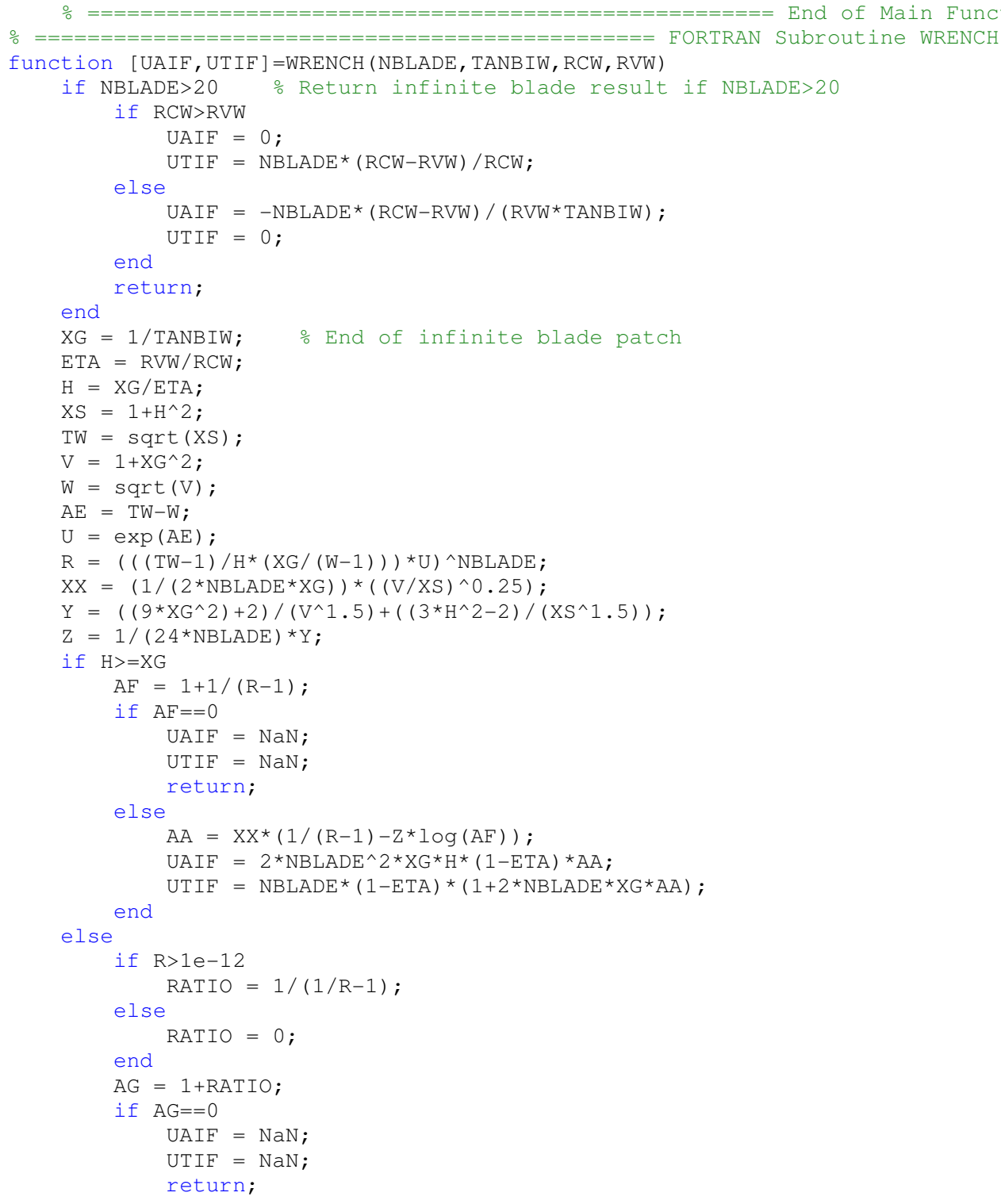


else

$\mathrm{AB}=-\mathrm{XX}$ * $\left(\mathrm{RATIO}+\mathrm{Z}^{\star} \log (\mathrm{AG})\right) ;$

$\mathrm{UAIF}=\mathrm{NBLADE} * \mathrm{XG} *(1-1 / \mathrm{ETA}) *(1-2 * \mathrm{NBLADE} * \mathrm{XG} * \mathrm{AB})$;

$\mathrm{UTIF}=2{ }^{*} \mathrm{NBLADE}^{\wedge} 2{ }^{*} \mathrm{XG}^{\star}(1-\mathrm{ETA}){ }^{*} \mathrm{AB}$;

end

end 


\section{Appendix B: AUV Propeller Design Results}




\section{B.1 AUV Propeller Chordlength Coordinates}

Table B-1: Chordlength distribution of an off-theshelf propeller.

$\begin{array}{cccc}\mathrm{R} & \mathrm{r} / \mathrm{R} & \mathrm{C} & \mathrm{C} / \mathrm{D} \\ 0.0200 & 0.0673 & 0.0488 & 0.0822 \\ 0.0300 & 0.1010 & 0.0459 & 0.0773 \\ 0.0400 & 0.1347 & 0.0445 & 0.0749 \\ 0.0500 & 0.1684 & 0.0440 & 0.0741 \\ 0.0600 & 0.2020 & 0.0443 & 0.0746 \\ 0.0700 & 0.2357 & 0.0452 & 0.0761 \\ 0.0800 & 0.2694 & 0.0469 & 0.0790 \\ 0.0900 & 0.3030 & 0.0488 & 0.0822 \\ 0.1000 & 0.3367 & 0.0506 & 0.0852 \\ 0.1100 & 0.3704 & 0.0507 & 0.0854 \\ 0.1200 & 0.4040 & 0.0498 & 0.0838 \\ 0.1300 & 0.4377 & 0.0485 & 0.0816 \\ 0.1400 & 0.4714 & 0.0472 & 0.0795 \\ 0.1500 & 0.5051 & 0.0461 & 0.0776 \\ 0.1600 & 0.5387 & 0.0445 & 0.0749 \\ 0.1700 & 0.5724 & 0.0430 & 0.0724 \\ 0.1800 & 0.6061 & 0.0414 & 0.0697 \\ 0.1900 & 0.6397 & 0.0398 & 0.0670 \\ 0.2000 & 0.6734 & 0.0379 & 0.0638 \\ 0.2100 & 0.7071 & 0.0359 & 0.0604 \\ 0.2200 & 0.7407 & 0.0338 & 0.0569 \\ 0.2300 & 0.7744 & 0.0318 & 0.0535 \\ 0.2400 & 0.8081 & 0.0294 & 0.0495 \\ 0.2500 & 0.8418 & 0.0270 & 0.0455 \\ 0.2600 & 0.8754 & 0.0240 & 0.0404 \\ 0.2700 & 0.9091 & 0.0212 & 0.0357 \\ 0.2800 & 0.9428 & 0.0180 & 0.0303 \\ 0.2900 & 0.9764 & 0.0125 & 0.0210 \\ 0.2950 & 0.9933 & 0.0062 & 0.0104 \\ 0.2970 & 1.0000 & 0.0010 & 0.0017\end{array}$

Table B-2: Chordlength distribution of an optimized AUV propeller.

$\begin{array}{ll}r / R & c / D \\ 0.2000 & 0.2269 \\ 0.2500 & 0.2193 \\ 0.3000 & 0.2148 \\ 0.4000 & 0.2029 \\ 0.5000 & 0.1885 \\ 0.6000 & 0.1693 \\ 0.7000 & 0.1545 \\ 0.8000 & 0.1275 \\ 0.9000 & 0.0801 \\ 0.9500 & 0.0572 \\ 1.0000 & 0.0032\end{array}$




\section{B.2 Geometry used for the US Navy 4148 Propeller Example}

$\begin{array}{ccccccl}r / R & \mathrm{P} / \mathrm{D} & \mathrm{C} / \mathrm{D} & \text { fo/C } & \text { to/C } & \text { rake } & \text { skew } \\ 0.204 & 1.11 & 0.162 & 0.0310 & 0.2010 & 0.0 & 0.000 \\ 0.236 & 1.11 & 0.176 & 0.0278 & 0.1676 & 0.0 & 0.000 \\ 0.298 & 1.10 & 0.202 & 0.0211 & 0.1191 & 0.0 & 0.000 \\ 0.384 & 1.10 & 0.217 & 0.0159 & 0.0939 & 0.0 & 0.000 \\ 0.487 & 1.11 & 0.230 & 0.0117 & 0.0717 & 0.0 & 0.000 \\ 0.600 & 1.11 & 0.231 & 0.0089 & 0.0541 & 0.0 & 0.000 \\ 0.713 & 1.12 & 0.227 & 0.0071 & 0.0469 & 0.0 & 0.000 \\ 0.816 & 1.13 & 0.214 & 0.0057 & 0.0405 & 0.0 & 0.000 \\ 0.902 & 1.13 & 0.179 & 0.0046 & 0.0331 & 0.0 & 0.000 \\ 0.964 & 1.14 & 0.121 & 0.0033 & 0.0288 & 0.0 & 0.000 \\ 0.996 & 1.14 & 0.045 & 0.0004 & 0.0039 & 0.0 & 0.000\end{array}$

\section{B.3 AUV basicPVL Parametric Analysis}

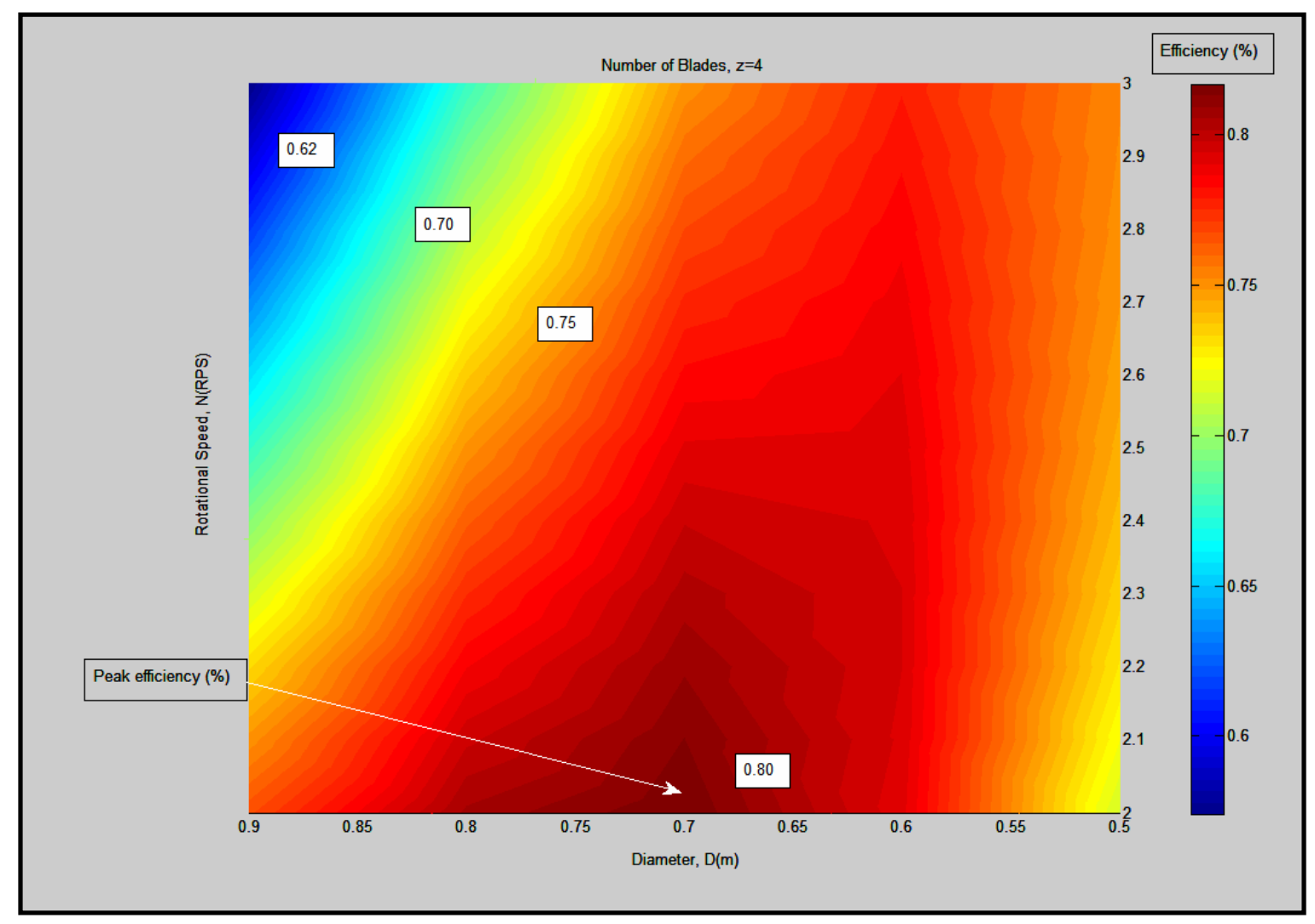

Figure B-1: Efficiency for a 4-bladed propeller. 


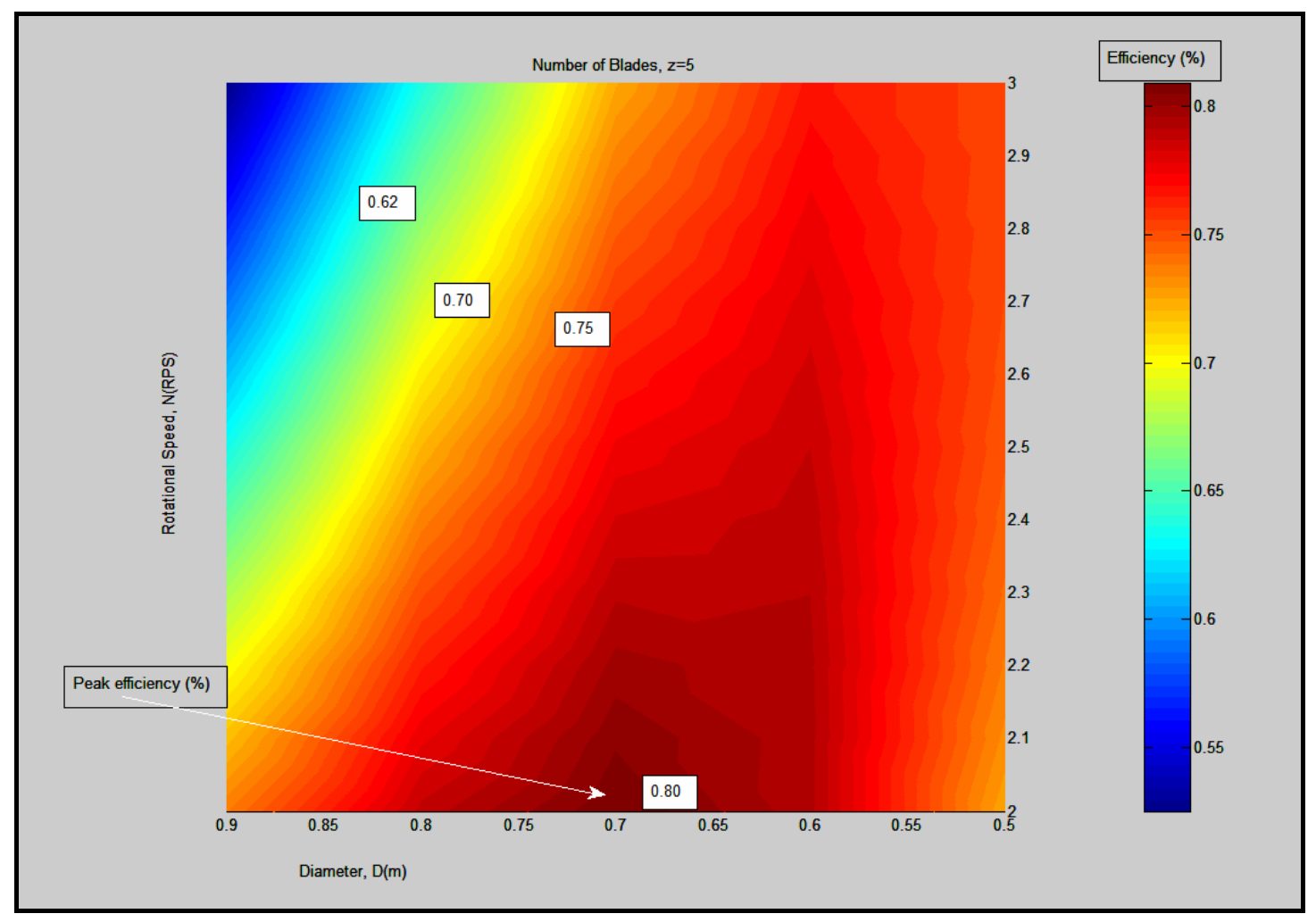

Figure B-2: Efficiency for a 5-bladed propeller.

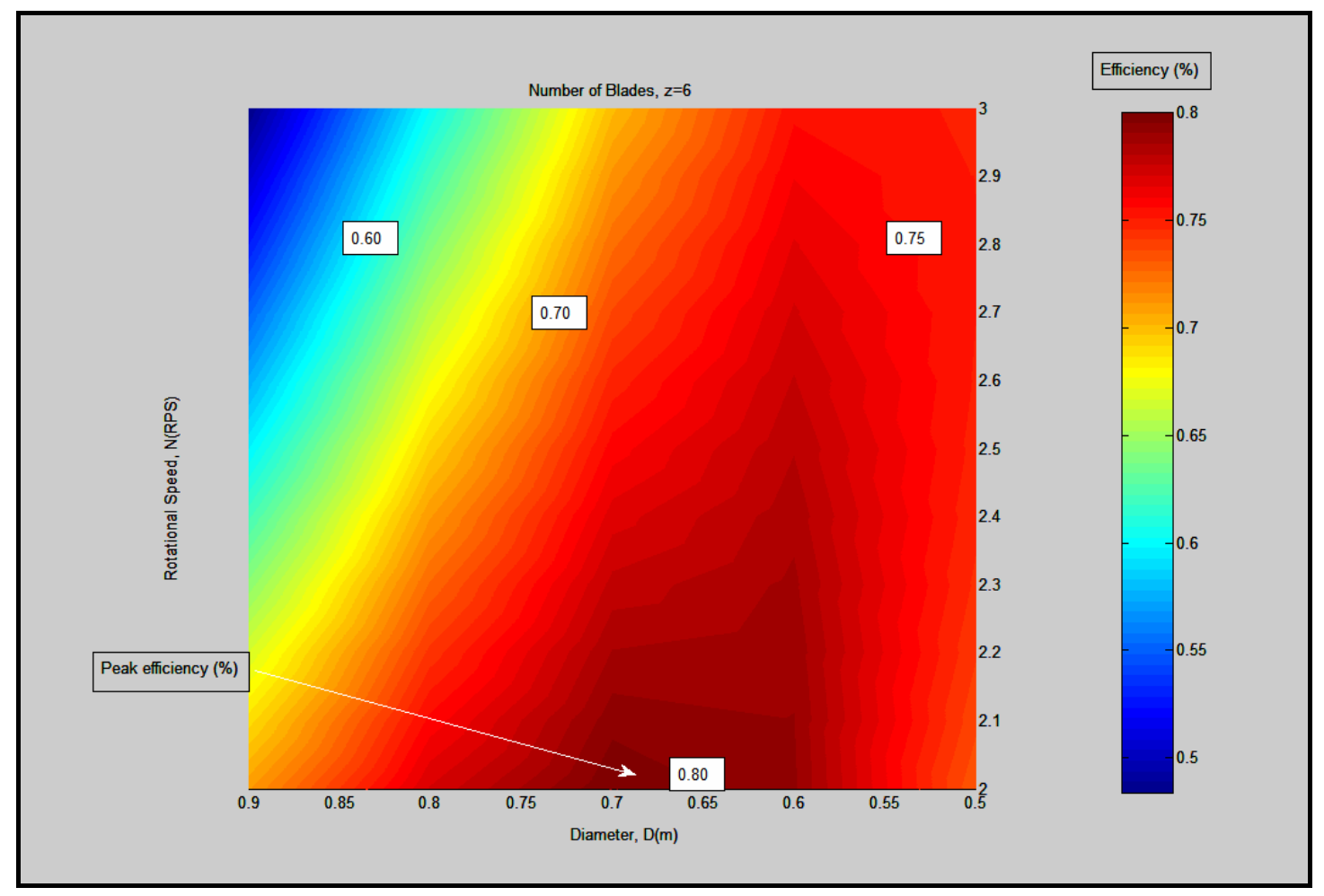

Figure B-3: Efficiency for a 6-bladed propeller. 


\section{B.4 AUV Off-the-Shelf Propellers Tow-Tank Testing}

Table B-3: Forward and reverse numerical values for the Bollard Test.
RUN PropRPM RPM
A
V T Force Force(lbs) Force(N)

fwdbol $=$

$\begin{array}{ccccccccc}1.0000 & 16.6500 & 5.0000 & 0.0152 & 46.6120 & 30.0000 & 1.0000 & 0.1987 & 0.8840 \\ 2.0000 & 33.3000 & 10.0000 & 0.0454 & 46.5900 & 29.0000 & 4.0000 & 0.7950 & 3.5362 \\ 3.0000 & 49.9500 & 15.0000 & 0.1227 & 46.5860 & 28.0400 & 9.0000 & 1.7887 & 7.9564 \\ 4.0000 & 66.6000 & 20.0000 & 0.2573 & 46.5540 & 27.0000 & 17.0000 & 3.3786 & 15.0287 \\ 5.0000 & 83.2500 & 25.0000 & 0.4859 & 46.5214 & 27.5000 & 27.0000 & 5.3660 & 23.8691 \\ 6.0000 & 99.9000 & 30.0000 & 0.8595 & 46.4040 & 28.7300 & 39.0000 & 7.7509 & 34.4775 \\ 7.0000 & 116.5500 & 35.0000 & 1.4248 & 46.2940 & 30.8400 & 53.0000 & 10.5332 & 46.8541 \\ 8.0000 & 133.2000 & 40.0000 & 2.1514 & 46.1220 & 34.7400 & 73.0000 & 14.5080 & 64.5349\end{array}$

revbol $=$

$\begin{array}{ccccccccc}1.0000 & 16.6500 & -5.0000 & 0.2376 & 46.5710 & 33.5800 & 1.0000 & 0.1987 & 0.8840 \\ 2.0000 & 33.3000 & -10.0000 & 0.3615 & 46.5400 & 33.9700 & 3.0000 & 0.5962 & 2.6521 \\ 3.0000 & 49.9500 & -15.0000 & 0.5044 & 46.5120 & 34.2700 & 6.0000 & 1.1924 & 5.3042 \\ 4.0000 & 66.6000 & -20.0000 & 0.7426 & 46.4510 & 34.7200 & 9.5000 & 1.8880 & 8.3984 \\ 5.0000 & 83.2500 & -25.0000 & 1.0988 & 46.3700 & 36.1300 & 15.0000 & 2.9811 & 13.2606 \\ 6.0000 & 99.9000 & -30.0000 & 1.4324 & 46.2960 & 42.0300 & 21.0000 & 4.1735 & 18.5648 \\ 7.0000 & 116.5500 & -35.0000 & 2.1383 & 46.1520 & 46.6800 & 28.0000 & 5.5647 & 24.7531 \\ 8.0000 & 133.2000 & -40.0000 & 3.0395 & 45.9380 & 47.7100 & 37.0000 & 7.3534 & 32.7095\end{array}$


Table B-4: Forward and reverse numerical values for the moving Cart Test.

RUN PropRPM RPM A $\quad$ V $\quad T$ Force Cart Spd(m/s) Force(lbs) Force(N)

fwdcart $=$

\begin{tabular}{|c|c|c|c|c|c|c|c|c|c|}
\hline & & & & & & & & & \\
\hline 2.0000 & & & & & & & & & \\
\hline & & & & & & & & & \\
\hline & & & & & & & & & \\
\hline & & & & & & & & & \\
\hline & & & & & & & & & \\
\hline & & & & & & & & & \\
\hline & & & & & & & & & \\
\hline & & & & & & & & & \\
\hline & & & & & & & & & \\
\hline & & & & & & & & & \\
\hline & & & & & & & & & \\
\hline & & & & & & & & & \\
\hline & & & & & & & & & \\
\hline & & & & & & & & & \\
\hline & & & & & & & & & \\
\hline & & & & & & & & & \\
\hline & & & & & & & & & \\
\hline & & & & & & & & & \\
\hline & & & & & & & & & \\
\hline & & & & & & & & & \\
\hline & & & & & & & & & \\
\hline & & & & & & & & & \\
\hline & & & & & & & & & \\
\hline & & & & & & & & & 51.2743 \\
\hline & & & & & & & & & 44.2020 \\
\hline & & & & & & & & & 35.3616 \\
\hline & & & & & & & & & \\
\hline & & & & & & & & & \\
\hline & & & & & & & & & 0 \\
\hline & & & & & & & & & 44200 \\
\hline
\end{tabular}

revcart $=$

$\begin{array}{cccccccccl}1.0000 & 83.2500 & 25.0000 & 0.9600 & 46.3940 & 32.2900 & 11.5000 & 0.2500 & 2.2855 & 10.1665 \\ 2.0000 & 83.2500 & 25.0000 & 0.9197 & 46.4170 & 42.1800 & 6.5000 & 0.3500 & 1.2918 & 5.7463 \\ 3.0000 & 83.2500 & 25.0000 & 0.8764 & 46.4350 & 42.6800 & -0.5000 & 0.4500 & 0.0994 & 0.4420 \\ 4.0000 & 99.9000 & 30.0000 & 1.4181 & 46.2980 & 36.9400 & 19.0000 & 0.2500 & 3.7761 & 16.7968 \\ 5.0000 & 99.9000 & 30.0000 & 1.5307 & 46.2880 & 43.5500 & 13.5000 & 0.3500 & 2.6830 & 11.9345 \\ 6.0000 & 99.9000 & 30.0000 & 1.7507 & 46.2190 & 47.3700 & 6.5000 & 0.4500 & 1.2918 & 5.7463 \\ 7.0000 & 99.9000 & 30.0000 & 1.2024 & 46.3470 & 47.5800 & -2.5000 & 0.5500 & 0.4968 & 2.2101 \\ 8.0000 & 99.9000 & 30.0000 & 1.3644 & 46.3310 & 48.0000 & 3.0000 & 0.4900 & 0.5962 & 2.6521 \\ 9.0000 & 116.5500 & 35.0000 & 2.3352 & 46.1090 & 47.5100 & 22.5000 & 0.3500 & 4.4716 & 19.8909 \\ 10.0000 & 116.5500 & 35.0000 & 2.3075 & 46.1220 & 35.5800 & 37.5000 & 0.2500 & 7.4527 & 33.1515 \\ 11.0000 & 116.5500 & 35.0000 & 2.0097 & 46.1860 & 42.8800 & 15.0000 & 0.4500 & 2.9811 & 13.2606 \\ 12.0000 & 116.5500 & 35.0000 & 1.9280 & 46.2030 & 47.8500 & 6.0000 & 0.5500 & 1.1924 & 5.3042 \\ 13.0000 & 116.5500 & 35.0000 & 1.9105 & 46.2120 & 50.0200 & -5.0000 & 0.6500 & 0.9937 & 4.4202\end{array}$




\section{B.5 Actual Measurements for the 3D Printed Propeller Blade}

\begin{tabular}{llllll}
\multicolumn{2}{l}{ Point $x$} & \multicolumn{3}{l}{ y $z_{f}$ fore $z_{\text {aft }}$} \\
1 & 2.5707 & 1.5464 & -0.0309 & -0.0398 \\
2 & 2.8907 & 0.8025 & -0.5636 & -0.6551 \\
3 & 3 & 0 & -1.1199 & -1.2514 & \\
4 & 2.8907 & -0.8025 & -1.778 & -1.8506 \\
5 & 2.5707 & -1.5464 & -1.9488 & -2.5876 \\
6 & 8.2315 & -1.6739 & -1.5567 & -1.9734 \\
7 & 8.3578 & -0.8412 & -1.2415 & -1.6567 \\
8 & 8.40 & -0.9632 & -1.3577 & \\
9 & 8.3578 & 0.8412 & -0.7938 & -1.0573 \\
10 & 8.2315 & 1.6739 & -0.8866 & -0.7805 \\
11 & 9.4948 & 1.4172 & -1.0506 & -0.9654 \\
12 & 9.5737 & 0.7105 & -1.2571 & -1.1636 \\
13 & 9.60 & -1.4902 & -1.3854 & \\
14 & 9.5737 & -0.7105 & -1.742 & -1.6094 \\
15 & 9.4948 & -1.4172 & -2.5339 & -1.8508
\end{tabular}

Table B-5: Actual Measurements of the 3D Printed Propeller. 


\section{Appendix C: MATLAB ${ }^{T M}$ Scripting for RHINOTM : OpenPVL_CADblade.txt}


! Curve

$0.551081,0.779187,0.970368$

$0.408407,0.854798,0.947187$ $0.307938,0.891982,0.871223$ $0.217712,0.915661,0.786151$ $0.134360,0.930011,0.695530$ $0.056403,0.937199,0.600818$ $-0.016790,0.938594,0.502699$ $-0.085518,0.935207,0.401566$ $-0.149205,0.927966,0.297235$ $-0.208660,0.917562,0.190392$ $-0.261703,0.905208,0.079957$ $-0.309369,0.891535,-0.033194$ $-0.352123,0.877104,-0.148589$ $-0.390713,0.862230,-0.265673$ $-0.425415,0.847278,-0.384213$ $-0.456820,0.832391,-0.505476$ $-0.483360,0.818753,-0.625879$ $-0.506512,0.806023,-0.741774$ $-0.528599,0.793120,-0.856066$ $-0.560211,0.773289,-0.967389$ $-0.541951,0.784945,-0.973347$ $-0.473148,0.824119,-0.871522$ $-0.396264,0.859941,-0.771845$ $-0.319915,0.888170,-0.674073$ $-0.244203,0.909611,-0.582370$ $-0.168731,0.924943,-0.494154$ $-0.093756,0.934495,-0.404805$ $-0.020636,0.938525,-0.315155$ $0.050020,0.937526,-0.224782$ $0.117458,0.932074,-0.133126$ $0.180721,0.922896,-0.039438$ $0.239116,0.910830,0.056967$ $0.294675,0.896017,0.155090$ $0.346629,0.879077,0.255596$ $0.395648,0.860197,0.358246$ $0.441581,0.839780,0.463350$ $0.484074,0.818372,0.571437$ $0.522135,0.796975,0.683684$ $0.553411,0.777696,0.803076$ $0.551081,0.779187,0.970368$ Curve

$0.561595,0.850421,0.957941$ $0.421788,0.927739,0.934312$ $0.321968,0.966924,0.859339$ $0.231925,0.992379,0.775449$ $0.148453,1.008249,0.686115$ $0.070141,1.016703,0.592759$ $-0.003612,1.019113,0.496051$ $-0.073089,1.016495,0.396375$ $-0.137742,1.009768,0.293545$ $-0.198340,0.999633,0.188234$ $-0.252801,0.987267,0.079372$ $-0.302112,0.973310,-0.032182$ $-0.346706,0.958332,-0.145974$ $-0.387294,0.942660,-0.261459$ $-0.424132,0.926670,-0.378415$ $-0.457792,0.910512,-0.498057$ $-0.486688,0.895399,-0.616984$ $-0.512225,0.881039,-0.731654$ $-0.536708,0.866343,-0.844802$ $-0.570325,0.844591,-0.954960$ $-0.552865,0.856122,-0.960923$ $-0.483651,0.897043,-0.860381$ $-0.406729,0.934439,-0.761993$ $-0.330337,0.964096,-0.665434$ $-0.254591,0.986807,-0.574723$ $-0.179041,1.003269,-0.487405$ $-0.103907,1.013809,-0.399020$ $-0.030516,1.018663,-0.310341$ $0.040547,1.018313,-0.220952$
$0.108566,1.013320,-0.130306$ $0.172621,1.004394,-0.037663$ $0.232063,0.992346,0.057636$ $0.288827,0.977335,0.154610$ $0.342184,0.959955,0.253907$ $0.392778,0.940388,0.355286$ $0.440468,0.919017,0.459046$ $0.484926,0.896355,0.565697$ $0.525225,0.873352,0.676378$ $0.559159,0.852025,0.793977$ $0.561595,0.850421,0.957941$ Curve

$0.581413,0.910851,0.933857$ $0.446833,0.983886,0.909289$ $0.348179,1.022967,0.836183$ $0.258442,1.049237,0.754535$ $0.174718,1.066379,0.667651$ $0.095722,1.076349,0.576889$ $0.020907,1.080395,0.482887$ $-0.049984,1.079441,0.386009$ $-0.116453,1.074304,0.286072$ $-0.179194,1.065636,0.183716$ $-0.236307,1.054443,0.077902$ $-0.288695,1.041319,-0.030549$ $-0.336727,1.026794,-0.141216$ $-0.381048,1.011184,-0.253579$ $-0.421876,0.994842,-0.367434$ $-0.459743,0.977920,-0.483905$ $-0.493042,0.961561,-0.599929$ $-0.523044,0.945577,-0.712166$ $-0.552018,0.928960,-0.823036$ $-0.589395,0.905706,-0.930891$ $-0.573431,0.915897,-0.936823$ $-0.503443,0.956157,-0.838714$ $-0.426444,0.992893,-0.742753$ $-0.349961,1.022359,-0.648478$ $-0.274125,1.045249,-0.559640$ $-0.198398,1.062228,-0.474025$ $-0.122934,1.073582,-0.387474$ $-0.049004,1.079486,-0.300652$ $0.022853,1.080356,-0.213153$ $0.091989,1.076675,-0.124454$ $0.157557,1.069049,-0.033841$ $0.218986,1.058176,0.059304$ $0.278031,1.044217,0.154032$ $0.334039,1.027672,0.250956$ $0.387598,1.008691,0.349837$ $0.438594,0.987586,0.450952$ $0.486744,0.964765,0.554773$ $0.531237,0.940998,0.662363$ $0.570143,0.917948,0.776421$ $0.581413,0.910851,0.933857$ Curve

$0.608651,1.001095,0.900030$ $0.480790,1.068405,0.873992$ $0.383599,1.107023,0.803361$ $0.294197,1.134061,0.724739$ $0.210069,1.152614,0.641190$ $0.130097,1.164355,0.553978$ $0.053806,1.170364,0.463697$ $-0.019029,1.171446,0.370684$ $-0.087974,1.168293,0.274762$ $-0.153626,1.161484,0.176524$ $-0.214327,1.151829,0.074977$ $-0.270864,1.139860,-0.029105$ $-0.323531,1.126044,-0.135353$ $-0.372883,1.110678,-0.243284$ $-0.419093,1.094079,-0.352716$ $-0.462637,1.076389,-0.464656$ $-0.501906,1.058649,-0.576487$ $-0.537997,1.040772,-0.685145$
$-0.573106,1.021859,-0.792637$ $-0.615645,0.996809,-0.897127$ $-0.601657,1.005314,-0.902934$ $-0.530466,1.044631,-0.808200$ $-0.453179,1.080406,-0.715568$ $-0.376384,1.109496,-0.624432$ $-0.300227,1.132480,-0.538188$ $-0.224074,1.149973,-0.454939$ $-0.148001,1.162215,-0.370933$ $-0.073193,1.169312,-0.286693$ $-0.000131,1.171600,-0.201833$ $0.070626,1.169470,-0.115866$ $0.138330,1.163405,-0.028112$ $0.202507,1.153966,0.061985$ $0.264681,1.141311,0.153527$ $0.324290,1.125825,0.247080$ $0.381857,1.107625,0.342411$ $0.437293,1.086932,0.439762$ $0.490376,1.064039,0.539557$ $0.540443,1.039504,0.642751$ $0.585912,1.014571,0.751780$ $0.608651,1.001095,0.900030$ Curve

$0.641804,1.119811,0.860044$ $0.521186,1.180785,0.831856$ $0.425496,1.218541,0.763840$ $0.336318,1.246105,0.688540$ $0.251574,1.265938,0.608721$ $0.170334,1.279404,0.525528$ $0.092201,1.287396,0.439501$ $0.016992,1.290581,0.350941$ $-0.054933,1.289524,0.259682$ $-0.124065,1.284717,0.166259$ $-0.189010,1.276779,0.069748$ $-0.250429,1.266165,-0.029146$ $-0.308541,1.253272,-0.130105$ $-0.363803,1.238360,-0.232694$ $-0.416344,1.221698,-0.336759$ $-0.466584,1.203407,-0.443168$ $-0.512963,1.184381,-0.549789$ $-0.556376,1.164618,-0.653887$ $-0.598877,1.143344,-0.757038$ $-0.647703,1.116409,-0.857267$ $-0.635906,1.123171,-0.862821$ $-0.562827,1.161514,-0.772129$ $-0.484646,1.196247,-0.683446$ $-0.406928,1.224866,-0.596045$ $-0.329848,1.247834,-0.512905$ $-0.252710,1.265712,-0.432476$ $-0.175503,1.278705,-0.351473$ $-0.099296,1.286868,-0.270278$ $-0.024500,1.290461,-0.188528$ $0.048425,1.289784,-0.105783$ $0.118841,1.285210,-0.021415$ $0.186379,1.277165,0.065066$ $0.252304,1.265793,0.152828$ $0.316162,1.251371,0.242382$ $0.378392,1.233981,0.333502$ $0.438936,1.213764,0.426396$ $0.497631,1.190904,0.521429$ $0.553961,1.165768,0.619436$ $0.606705,1.139209,0.722544$ $0.641804,1.119811,0.860044$ Curve

$0.678170,1.265770,0.815817$ $0.564253,1.320495,0.785121$ $0.469745,1.356992,0.719793$ $0.380492,1.384671,0.647983$ $0.294839,1.405404,0.572126$ $0.212041,1.420256,0.493234$ $0.131779,1.429938,0.411784$ 
$0.053907,1.434985,0.328041$ $-0.021284,1.435840,0.241854$ $-0.094183,1.432906,0.153692$ $-0.163660,1.426641,0.062717$ $-0.230250,1.417418,-0.030441$ $-0.294103,1.405558,-0.125524$ $-0.355580,1.391277,-0.222148$ $-0.414770,1.374793,-0.320191$ $-0.472032,1.356199,-0.420382$ $-0.525898,1.336233,-0.521070$ $-0.577069,1.314945,-0.619870$ $-0.627416,1.291680,-0.717937$ $-0.682975,1.263184,-0.813239$ $-0.673365,1.268333,-0.818394$ $-0.597971,1.305572,-0.732119$ $-0.518461,1.339137,-0.647699$ $-0.439372,1.367129,-0.564347$ $-0.360895,1.389908,-0.484614$ $-0.282309,1.407974,-0.407299$ $-0.203530,1.421501,-0.329597$ $-0.125498,1.430503,-0.251757$ $-0.048553,1.435176,-0.173448$ $0.026938,1.435745,-0.094280$ $0.100452,1.432480,-0.013686$ $0.171715,1.425694,0.068748$ $0.241742,1.415503,0.152278$ $0.310177,1.402098,0.237348$ $0.377382,1.385522,0.323748$ $0.443322,1.365853,0.411648$ $0.507886,1.343183,0.501353$ $0.570703,1.317720,0.593565$ $0.630883,1.289991,0.690083$ $0.678170,1.265770,0.815817$ Curve

$0.710457,1.439441,0.764090$ $0.600831,1.488536,0.734224$ $0.506600,1.523186,0.673090$ $0.416699,1.550194,0.605993$ $0.329784,1.570981,0.535154$ $0.245247,1.586377,0.461498$ $0.162827,1.596943,0.385461$ $0.082407,1.603106,0.307283$ $0.004231,1.605217,0.226821$ $-0.072017,1.603606,0.144503$ $-0.145390,1.598624,0.059542$ $-0.216332,1.590578,-0.027483$ $-0.284949,1.579729,-0.116347$ $-0.351540,1.566256,-0.206699$ $-0.416168,1.550336,-0.298432$ $-0.479169,1.532036,-0.392179$ $-0.539148,1.511972,-0.486604$ $-0.596636,1.490223,-0.579562$ $-0.653360,1.466240,-0.671930$ $-0.714519,1.437429,-0.761611$ $-0.706395,1.441438,-0.766569$ $-0.628389,1.477114,-0.685715$ $-0.546912,1.509180,-0.606651$ $-0.465845,1.536140,-0.528506$ $-0.385434,1.558261,-0.453534$ $-0.304960,1.575988,-0.380753$ $-0.224247,1.589482,-0.307693$ $-0.144141,1.598738,-0.234512$ $-0.064927,1.603909,-0.160899$ $0.013093,1.605169,-0.086496$ $0.089482,1.602726,-0.010776$ $0.164027,1.596820,0.066630$ $0.237574,1.587544,0.145027$ $0.309835,1.575037,0.224818$ $0.381117,1.559323,0.305800$ $0.451400,1.540447,0.388123$ $0.520609,1.518455,0.472051$
$0.588462,1.493470,0.558206$ $0.654289,1.465825,0.648181$ $0.710457,1.439441,0.764090$ Curve

$0.735751,1.638048,0.707402$ $0.629829,1.681621,0.679556$ $0.536032,1.713827,0.623236$ $0.445792,1.739483,0.561403$ $0.358025,1.759645,0.496094$ $0.272233,1.774943,0.428162$ $0.188209,1.785808,0.358008$ $0.105857,1.792575,0.285850$ $0.025387,1.795519,0.211551$ $-0.053460,1.794902,0.135504$ $-0.129887,1.790995,0.056967$ $-0.204256,1.784044,-0.023525$ $-0.276650,1.774260,-0.105771$ $-0.347312,1.761791,-0.189445$ $-0.416294,1.746777,-0.274452$ $-0.483917,1.729265,-0.361343$ $-0.548868,1.709759,-0.449016$ $-0.611525,1.688363,-0.535536$ $-0.673473,1.664622,-0.621575$ $-0.739220,1.636486,-0.705008$ $-0.732282,1.639602,-0.709795$ $-0.652076,1.673120,-0.635002$ $-0.568899,1.703199,-0.561954$ $-0.486126,1.728645,-0.489702$ $-0.404072,1.749645,-0.420242$ $-0.322038,1.766585,-0.352758$ $-0.239758,1.779620,-0.285060$ $-0.157995,1.788734,-0.217239$ $-0.076988,1.794047,-0.149004$ $0.003015,1.795696,-0.080025$ $0.081650,1.793841,-0.009809$ $0.158748,1.788668,0.061979$ $0.235023,1.780252,0.134682$ $0.310240,1.768695,0.208670$ $0.384661,1.754015,0.283750$ $0.458277,1.736236,0.360052$ $0.531037,1.715381,0.437811$ $0.602725,1.691525,0.517590$ $0.672836,1.664880,0.600822$ $0.735751,1.638048,0.707402$ Curve

$0.752770,1.857699,0.648862$ $0.650655,1.895879,0.622932$ $0.557858,1.925228,0.571419$ $0.467937,1.949036,0.514882$ $0.380031,1.968066,0.455165$ $0.293745,1.982781,0.393042$ $0.208910,1.993505,0.328875$ $0.125455,2.000492,0.262862$ $0.043556,2.003949,0.194873$ $-0.036997,2.004080,0.125265$ $-0.115537,2.001089,0.053349$ $-0.192361,1.995170,-0.020386$ $-0.267528,1.986488,-0.095764$ $-0.341235,1.975162,-0.172488$ $-0.413521,1.961303,-0.250476$ $-0.484687,1.944938,-0.330199$ $-0.553521,1.926479,-0.410770$ $-0.620273,1.906035,-0.490460$ $-0.686378,1.883240,-0.569767$ $-0.755694,1.856511,-0.646600$ $-0.749846,1.858881,-0.651124$ $-0.668276,1.889739,-0.582547$ $-0.584197,1.917400,-0.515630$ $-0.500506,1.940928,-0.449395$ $-0.417561,1.960446,-0.385592$ $-0.334696,1.976281,-0.323554$
$-0.251589,1.988570,-0.261360$ $-0.168914,1.997292,-0.199050$ $-0.086871,2.002539,-0.136354$ $-0.005661,2.004414,-0.072971$ $0.074417,2.003040,-0.008448$ $0.153234,1.998556,0.057512$ $0.231383,1.991022,0.124303$ $0.308676,1.980512,0.192260$ $0.385334,1.967035,0.261198$ $0.461356,1.950604,0.331233$ $0.536710,1.931230,0.402570$ $0.611237,1.908952,0.475710$ $0.684570,1.883898,0.551922$ $0.752770,1.857699,0.648862$ Curve

$0.760832,2.094175,0.590743$ $0.662764,2.127247,0.566203$ $0.571642,2.153523,0.519200$ $0.482798,2.175165,0.467719$ $0.395565,2.192707,0.413393$ $0.309632,2.206482,0.356910$ $0.224870,2.216725,0.298589$ $0.141224,2.223621,0.238607$ $0.058839,2.227324,0.176844$ $-0.022449,2.227988,0.113617$ $-0.102099,2.225761,0.048304$ $-0.180345,2.220791,-0.018660$ $-0.257225,2.213204,-0.087126$ $-0.332899,2.203092,-0.156830$ $-0.407389,2.190541,-0.227703$ $-0.480972,2.175569,-0.300146$ $-0.552550,2.158500,-0.373471$ $-0.622278,2.139440,-0.446162$ $-0.691430,2.118103,-0.518558$ $-0.763243,2.093298,-0.588674$ $-0.758422,2.095049,-0.592813$ $-0.676455,2.122933,-0.530323$ $-0.592421,2.147900,-0.469352$ $-0.508745,2.169243,-0.408955$ $-0.425792,2.187038,-0.350643$ $-0.342946,2.201550,-0.293893$ $-0.259861,2.212896,-0.237052$ $-0.177125,2.221050,-0.180115$ $-0.094900,2.226079,-0.122837$ $-0.013345,2.228061,-0.064951$ $0.067299,2.227085,-0.006051$ $0.146938,2.223251,0.054122$ $0.226051,2.216605,0.115021$ $0.304494,2.207197,0.176943$ $0.382447,2.195033,0.239718$ $0.459917,2.180117,0.303444$ $0.536888,2.162449,0.368295$ $0.613250,2.142046,0.434703$ $0.688751,2.118975,0.503762$ $0.760832,2.094175,0.590743$ Curve

$0.758333,2.343572,0.533562$ $0.664551,2.371871,0.510363$ $0.575839,2.394955,0.467709$ $0.488908,2.414201,0.421123$ $0.403251,2.429977,0.372028$ $0.318628,2.442514,0.321027$ $0.234939,2.451979,0.268400$ $0.152144,2.458506,0.214299$ $0.070360,2.462204,0.158620$ $-0.010541,2.463186,0.101638$ $-0.090121,2.461560,0.042800$ $-0.168565,2.457434,-0.017509$ $-0.245896,2.450905,-0.079167$ $-0.322239,2.442040,-0.141944$ $-0.397607,2.430907,-0.205785$ 
$-0.472245,2.417516,-0.271026$ $-0.545180,2.402119,-0.337147$ $-0.616496,2.384813,-0.402835$ $-0.687307,2.365377,-0.468301$ $-0.760298,2.342935,-0.531708$ $-0.756368,2.344207,-0.535417$ $-0.675057,2.368902,-0.478895$ $-0.592062,2.390996,-0.423728$ $-0.509390,2.409963,-0.369042$ $-0.427394,2.425847,-0.316128$ $-0.345515,2.438856,-0.264588$ $-0.263408,2.449084,-0.213011$ $-0.181577,2.456507,-0.161361$ $-0.100153,2.461172,-0.109420$ $-0.019261,2.463134,-0.056955$ $0.060907,2.462456,-0.003605$ $0.140284,2.459211,0.050849$ $0.219255,2.453431,0.105925$ $0.297707,2.445152,0.161882$ $0.375791,2.434374,0.218565$ $0.453516,2.421099,0.276057$ $0.530876,2.405321,0.334504$ $0.607801,2.387043,0.394269$ $0.684127,2.366298,0.456283$ $0.758333,2.343572,0.533562$ Curve

$0.738257,2.603385,0.473866$ $0.649941,2.626826,0.452099$ $0.565210,2.646351,0.413929$ $0.481847,2.662792,0.372394$ $0.399471,2.676389,0.328704$ $0.317900,2.687299,0.283373$ $0.237061,2.695633,0.236640$ $0.156922,2.701483,0.188636$ $0.077577,2.704925,0.139269$ $-0.001070,2.706037,0.088774$ $-0.078675,2.704893,0.036675$ $-0.155382,2.701572,-0.016698$ $-0.231200,2.696142,-0.071250$ $-0.306223,2.688655,-0.126787$ $-0.380461,2.679158,-0.183265$ $-0.454118,2.667660,-0.240966$ $-0.526357,2.654352,-0.299518$ $-0.597217,2.639312,-0.357805$ $-0.667645,2.622382,-0.415934$ $-0.739819,2.602941,-0.472261$ $-0.736696,2.603827,-0.475471$ $-0.657875,2.624850,-0.425137$ $-0.577720,2.643648,-0.375966$ $-0.497845,2.659847,-0.327188$ $-0.418568,2.673469,-0.279892$ $-0.339398,2.684669,-0.233790$ $-0.260012,2.693516,-0.187704$ $-0.180836,2.699988,-0.141574$ $-0.101971,2.704115,-0.095212$ $-0.023514,2.705935,-0.048420$ $0.054385,2.705490,-0.000886$ $0.131681,2.702831,0.047570$ $0.208675,2.697979,0.096536$ $0.285283,2.690957,0.146230$ $0.361625,2.681765,0.196519$ $0.437712,2.670402,0.247467$ $0.513548,2.656860,0.299193$ $0.589090,2.641138,0.351993$ $0.664246,2.623245,0.406630$ $0.738257,2.603385,0.473866$ Curve

$0.717675,2.864212,0.423219$ $0.633930,2.883904,0.403005$ $0.552740,2.900560,0.368727$ $0.472617,2.914687,0.331528$
$0.393273,2.926449,0.292453$ $0.314568,2.935952,0.251946$ $0.236445,2.943274,0.210215$ $0.158883,2.948478,0.167372$ $0.081955,2.951618,0.123339$ $0.005590,2.952751,0.078317$ $-0.069938,2.951928,0.031890$ $-0.144739,2.949206,-0.015653$ $-0.218819,2.944637,-0.064237$ $-0.292248,2.938258,-0.113695$ $-0.365032,2.930106,-0.163992$ $-0.437350,2.920187,-0.215368$ $-0.508470,2.908647,-0.267551$ $-0.578401,2.895552,-0.319579$ $-0.647960,2.880784,-0.371492$ $-0.718944,2.863894,-0.421811$ $-0.716406,2.864530,-0.424626$ $-0.639992,2.882564,-0.379587$ $-0.562494,2.898684,-0.335562$ $-0.485243,2.912612,-0.291864$ $-0.408531,2.924358,-0.249425$ $-0.331922,2.934041,-0.208035$ $-0.255117,2.941714,-0.166691$ $-0.178476,2.947357,-0.125321$ $-0.102083,2.950991,-0.083761$ $-0.026011,2.952641,-0.041839$ $0.049619,2.952339,0.000717$ $0.124777,2.950118,0.044058$ $0.199703,2.945995,0.087828$ $0.274334,2.939984,0.132212$ $0.348766,2.932086,0.177095$ $0.423013,2.922298,0.222527$ $0.497084,2.910614,0.268608$ $0.570953,2.897029,0.315584$ $0.644574,2.881543,0.364094$ $0.717675,2.864212,0.423219$ Curve

$0.714271,3.118727,0.389780$ $0.632399,3.136353,0.371044$ $0.552413,3.151425,0.339482$ $0.473304,3.164273,0.305242$ $0.394843,3.175018,0.269280$ $0.316918,3.183740,0.232003$ $0.239482,3.190500,0.193600$ $0.162520,3.195345,0.154173$ $0.086098,3.198316,0.113649$ $0.010154,3.199459,0.072212$ $-0.065074,3.198813,0.029478$ $-0.139677,3.196425,-0.014289$ $-0.213658,3.192333,-0.059020$ $-0.287077,3.186570,-0.104561$ $-0.359937,3.179164,-0.150881$ $-0.432409,3.170120,-0.198189$ $-0.503823,3.159557,-0.246258$ $-0.574154,3.147537,-0.294218$ $-0.644145,3.133962,-0.342084$ $-0.715380,3.118472,-0.388464$ $-0.713162,3.118981,-0.391095$ $-0.637159,3.135390,-0.349671$ $-0.560202,3.150050,-0.309202$ $-0.483480,3.162734,-0.269022$ $-0.407286,3.173446,-0.229964$ $-0.331222,3.182284,-0.191847$ $-0.254983,3.189298,-0.153774$ $-0.178895,3.194470,-0.115669$ $-0.103027,3.197816,-0.077382$ $-0.027441,3.199357,-0.038754$ $0.047763,3.199118,0.000465$ $0.122560,3.197127,0.040412$ $0.197159,3.193394,0.080758$ $0.271507,3.187934,0.121672$
$0.345689,3.180745,0.163047$ $0.419719,3.171825,0.204929$ $0.493605,3.161170,0.247407$ $0.567335,3.148773,0.290708$ $0.640882,3.134631,0.335414$ $0.714271,3.118727,0.389780$ Curve

$0.711613,3.367945,0.361948$ $0.631170,3.383944,0.344581$ $0.552107,3.397739,0.315311$ $0.473777,3.409543,0.283551$ $0.395996,3.419450,0.250188$ $0.318670,3.427521,0.215602$ $0.241764,3.433803,0.179967$ $0.165266,3.438333,0.143377$ $0.089235,3.441146,0.105762$ $0.013619,3.442276,0.067296$ $-0.061371,3.441756,0.027615$ $-0.135813,3.439623,-0.013035$ $-0.209707,3.435909,-0.054588$ $-0.283107,3.430641,-0.096897$ $-0.356015,3.423843,-0.139934$ $-0.428597,3.415517,-0.183883$ $-0.500230,3.405763,-0.228543$ $-0.570864,3.394638,-0.273117$ $-0.641180,3.382061,-0.317610$ $-0.712607,3.367735,-0.360698$ $-0.710620,3.368155,-0.363198$ $-0.634905,3.383245,-0.324835$ $-0.558326,3.396722,-0.287390$ $-0.481974,3.408394,-0.250204$ $-0.406151,3.418258,-0.214031$ $-0.330484,3.426402,-0.178704$ $-0.254665,3.432870,-0.143406$ $-0.178989,3.437646,-0.108066$ $-0.103517,3.440746,-0.072541$ $-0.028302,3.442187,-0.036684$ $0.046571,3.441988,-0.000263$ $0.121083,3.440173,0.036853$ $0.195418,3.436752,0.074351$ $0.269532,3.431735,0.112391$ $0.343500,3.425121,0.150870$ $0.417336,3.416911,0.189832$ $0.491050,3.407098,0.229361$ $0.564634,3.395679,0.269670$ $0.638078,3.382648,0.311305$ $0.711613,3.367945,0.361948$ Curve

$0.706412,3.608924,0.337282$ $0.627458,3.623485,0.321047$ $0.549485,3.636126,0.293757$ $0.472125,3.646978,0.264156$ $0.395231,3.656110,0.233067$ $0.318726,3.663572,0.200839$ $0.242583,3.669401,0.167637$ $0.166792,3.673626,0.133547$ $0.091407,3.676274,0.098501$ $0.016386,3.677374,0.062661$ $-0.058087,3.676952,0.025687$ $-0.132076,3.675038,-0.012195$ $-0.205582,3.671660,-0.050918$ $-0.278651,3.666838,-0.090346$ $-0.351284,3.660594,-0.130450$ $-0.423644,3.652927,-0.171393$ $-0.495151,3.643923,-0.212991$ $-0.565735,3.633633,-0.254515$ $-0.636023,3.621992,-0.295967$ $-0.707308,3.608748,-0.336096$ $-0.705517,3.609099,-0.338468$ $-0.630353,3.622983,-0.302836$ $-0.554403,3.635380,-0.268088$ 
$-0.478671,3.646124,-0.233572$ $-0.403463,3.655211,-0.199968$ $-0.328434,3.662715,-0.167120$ $-0.253272,3.668678,-0.134285$ $-0.178248,3.673088,-0.101394$ $-0.103415,3.675956,-0.068316$ $-0.028819,3.677298,-0.034912$ $0.045469,3.677129,-0.000968$ $0.119433,3.675471,0.033640$ $0.193236,3.672330,0.068618$ $0.266842,3.667716,0.104113$ $0.340319,3.661630,0.140029$ $0.413678,3.654069,0.176410$ $0.486932,3.645030,0.213332$ $0.560076,3.634510,0.250999$ $0.633109,3.622502,0.289924$ $0.706412,3.608924,0.337282$ Curve

$0.696795,3.838356,0.314484$ $0.619673,3.851559,0.299011$ $0.543213,3.863085,0.273427$ $0.467260,3.873005,0.245729$ $0.391699,3.881375,0.216670$ $0.316469,3.888232,0.186571$ $0.241546,3.893605,0.155579$ $0.166925,3.897517,0.123775$ $0.092655,3.899989,0.091093$ $0.018698,3.901045,0.057684$ $-0.054781,3.900705,0.023232$ $-0.127838,3.898995,-0.012055$ $-0.200472,3.895936,-0.048115$ $-0.272723,3.891545,-0.084820$ $-0.344593,3.885841,-0.122143$ $-0.416236,3.878821,-0.160226$ $-0.487121,3.870557,-0.198900$ $-0.557165,3.861097,-0.237510$ $-0.626937,3.850383,-0.276057$ $-0.697593,3.838211,-0.313378$ $-0.695998,3.838501,-0.315589$ $-0.621871,3.851205,-0.282469$ $-0.547037,3.862545,-0.250183$ $-0.472410,3.872381,-0.218104$ $-0.398287,3.880705,-0.186835$ $-0.324356,3.887582,-0.156230$ $-0.250309,3.893051,-0.125625$ $-0.176394,3.897100,-0.094955$ $-0.102654,3.899739,-0.064100$ $-0.029128,3.900981,-0.032932$ $0.044122,3.900840,-0.001256$ $0.117083,3.899333,0.031045$ $0.189902,3.896465,0.063694$ $0.262546,3.892245,0.096831$ $0.335076,3.886673,0.130365$ $0.407504,3.879748,0.164336$ $0.479840,3.871467,0.198817$ $0.552084,3.861827,0.233996$ $0.624244,3.850821,0.270351$ $0.696795,3.838356,0.314484$ Curve

$0.672534,4.054413,0.288880$ $0.598742,4.065965,0.273860$ $0.525362,4.076096,0.250036$ $0.452390,4.084839,0.224366$ $0.379737,4.092232,0.197507$ $0.307354,4.098305,0.169738$ $0.235225,4.103076,0.141189$ $0.163345,4.106566,0.111929$ $0.091756,4.108789,0.081901$ $0.020428,4.109763,0.051235$ $-0.050502,4.109503,0.019656$ $-0.121077,4.108030,-0.012657$
$-0.191297,4.105359,-0.045647$ $-0.261195,4.101505,-0.079205$ $-0.330772,4.096481,-0.113305$ $-0.400163,4.090286,-0.148068$ $-0.468906,4.082976,-0.183356$ $-0.536915,4.074591,-0.218599$ $-0.604688,4.065085,-0.253792$ $-0.673211,4.054301,-0.287905$ $-0.671858,4.054525,-0.289855$ $-0.600383,4.065724,-0.259452$ $-0.528305,4.075716,-0.229787$ $-0.456411,4.084392,-0.200298$ $-0.384969,4.091744,-0.171505$ $-0.313705,4.097823,-0.143285$ $-0.242341,4.102662,-0.115064$ $-0.171092,4.106251,-0.086784$ $-0.099991,4.108597,-0.058340$ $-0.029074,4.109711,-0.029619$ $0.041612,4.109603,-0.000450$ $0.112057,4.108286,0.029266$ $0.182383,4.105765,0.059290$ $0.252564,4.102046,0.089740$ $0.322652,4.097129,0.120539$ $0.392657,4.091013,0.151721$ $0.462591,4.083696,0.183349$ $0.532455,4.075176,0.215587$ $0.602263,4.065445,0.248851$ $0.672534,4.054413,0.288880$ Curve

$0.628739,4.254078,0.258738$ $0.560231,4.263641,0.244431$ $0.491962,4.272056,0.222751$ $0.424012,4.279335,0.199521$ $0.356315,4.285502,0.175289$ $0.288833,4.290579,0.150290$ $0.221554,4.294578,0.124635$ $0.154475,4.297514,0.098379$ $0.087630,4.299397,0.071476$ $0.020996,4.300238,0.044036$ $-0.045316,4.300051,0.015824$ $-0.111341,4.298848,-0.013006$ $-0.177078,4.296642,-0.042409$ $-0.242550,4.293444,-0.072293$ $-0.307761,4.289263,-0.102637$ $-0.372823,4.284098,-0.133541$ $-0.437347,4.277992,-0.164900$ $-0.501256,4.270976,-0.196235$ $-0.564967,4.263016,-0.227532$ $-0.629291,4.253996,-0.257916$ $-0.628186,4.254159,-0.259561$ $-0.561444,4.263481,-0.232314$ $-0.494209,4.271797,-0.205688$ $-0.427129,4.279025,-0.179208$ $-0.360434,4.285158,-0.153307$ $-0.293892,4.290235,-0.127890$ $-0.227265,4.294280,-0.102479$ $-0.160730,4.297285,-0.077019$ $-0.094318,4.299255,-0.051423$ $-0.028053,4.300198,-0.025597$ $0.038025,4.300121,0.000604$ $0.103910,4.299034,0.027264$ $0.169700,4.296940,0.054178$ $0.235375,4.293843,0.081450$ $0.300978,4.289744,0.109010$ $0.366519,4.284642,0.136889$ $0.432006,4.278535,0.165138$ $0.497445,4.271421,0.193894$ $0.562852,4.263295,0.223501$ $0.628739,4.254078,0.258738$ Curve

$0.559166,4.434399,0.221884$
$0.498505,4.441627,0.209402$ $0.437958,4.448005,0.190721$ $0.377662,4.453530,0.170735$ $0.317565,4.458218,0.149907$ $0.257641,4.462082,0.128435$ $0.197879,4.465132,0.106410$ $0.138278,4.467375,0.083880$ $0.078866,4.468818,0.060805$ $0.019626,4.469471,0.037277$ $-0.039353,4.469341,0.013099$ $-0.098097,4.468438,-0.011602$ $-0.156605,4.466770,-0.036785$ $-0.214897,4.464345,-0.062373$ $-0.272975,4.461170,-0.088347$ $-0.330936,4.457246,-0.114789$ $-0.388452,4.452602,-0.141611$ $-0.445452,4.447261,-0.168414$ $-0.502285,4.441201,-0.195186$ $-0.559623,4.434341,-0.221184$ $-0.558709,4.434456,-0.222583$ $-0.499365,4.441530,-0.199255$ $-0.439612,4.447842,-0.176459$ $-0.379990,4.453332,-0.153783$ $-0.320702,4.457994,-0.131586$ $-0.261552,4.461855,-0.109786$ $-0.202332,4.464932,-0.087985$ $-0.143192,4.467220,-0.066139$ $-0.084154,4.468722,-0.044173$ $-0.025239,4.469443,-0.022008$ $0.033520,4.469389,0.000478$ $0.092121,4.468565,0.023355$ $0.150643,4.466975,0.046452$ $0.209071,4.464622,0.069853$ $0.267440,4.461506,0.093502$ $0.325759,4.457627,0.117423$ $0.384035,4.452985,0.141661$ $0.442273,4.447578,0.166332$ $0.500491,4.441404,0.191728$ $0.559166,4.434399,0.221884$ Curve

$0.413872,4.596223,0.159362$ $0.369029,4.600040,0.150646$ $0.324239,4.603414,0.137367$ $0.279629,4.606339,0.123119$ $0.235162,4.608823,0.108243$ $0.190820,4.610872,0.092887$ $0.146595,4.612490,0.077119$ $0.102487,4.613681,0.060974$ $0.058515,4.614448,0.044423$ $0.014667,4.614795,0.027535$ $-0.028992,4.614728,0.010162$ $-0.072481,4.614250,-0.007598$ $-0.115799,4.613366,-0.025720$ $-0.158960,4.612080,-0.044147$ $-0.201963,4.610397,-0.062867$ $-0.244880,4.608317,-0.081943$ $-0.287466,4.605857,-0.101320$ $-0.329671,4.603028,-0.120701$ $-0.371752,4.599821,-0.140062$ $-0.414200,4.596193,-0.158849$ $-0.413543,4.596252,-0.159876$ $-0.369653,4.599990,-0.143052$ $-0.325471,4.603327,-0.126612$ $-0.281385,4.606232,-0.110261$ $-0.237541,4.608701,-0.094270$ $-0.193791,4.610748,-0.078588$ $-0.149986,4.612381,-0.062921$ $-0.106235,4.613596,-0.047229$ $-0.062554,4.614395,-0.031457$ $-0.018960,4.614780,-0.015547$ $0.024527,4.614754,0.000593$ 
$0.067903,4.614319,0.017012$ $0.111226,4.613478,0.033585$ $0.154486,4.612232,0.050374$ $0.197707,4.610582,0.067337$ $0.240896,4.608527,0.084491$ $0.284061,4.606068,0.101867$ $0.327205,4.603204,0.119548$ $0.370346,4.599934,0.137741$ $0.413872,4.596223,0.159362$ Curve

$0.345491,4.721287,0.129890$ $0.308256,4.723865,0.122178$ $0.271017,4.726147,0.111069$ $0.233900,4.728130,0.099245$ $0.196883,4.729816,0.086960$ $0.159953,4.731208,0.074322$ $0.123106,4.732311,0.061380$ $0.086342,4.733124,0.048162$ $0.049676,4.733651,0.034645$ $0.013098,4.733893,0.020878$ $-0.023343,4.733854,0.006754$ $-0.059661,4.733536,-0.007657$ $-0.095855,4.732941,-0.022334$ $-0.131935,4.732073,-0.037233$ $-0.167903,4.730933,-0.052344$ $-0.203813,4.729522,-0.067708$ $-0.239484,4.727850,-0.083279$ $-0.274873,4.725925,-0.098840$ $-0.310169,4.723739,-0.114385$ $-0.345736,4.721269,-0.129501$ $-0.345247,4.721305,-0.130279$ $-0.308604,4.723842,-0.116652$ $-0.271740,4.726106,-0.103323$ $-0.234951,4.728077,-0.090059$ $-0.198351,4.729754,-0.077050$ $-0.161833,4.731144,-0.064250$ $-0.125279,4.732254,-0.051447$ $-0.088770,4.733079,-0.038615$ $-0.052317,4.733622,-0.025713$ $-0.015930,4.733885,-0.012697$ $0.020373,4.733868,0.000498$ $0.056592,4.733573,0.013913$ $0.092770,4.733002,0.027451$ $0.128898,4.732156,0.041160$ $0.164995,4.731035,0.055009$ $0.201065,4.729640,0.069010$ $0.237115,4.727969,0.083189$ $0.273146,4.726025,0.097611$ $0.309172,4.723805,0.112437$ $0.345491,4.721287,0.129890$ Curve

$0.308007,4.815073,0.113751$ $0.275182,4.817061,0.104857$ $0.242329,4.818825,0.094183$ $0.209534,4.820362,0.083143$ $0.176786,4.821675,0.071867$ $0.144080,4.822763,0.060411$ $0.111415,4.823628,0.048800$ $0.078791,4.824271,0.037049$ $0.046215,4.824693,0.025143$ $0.013683,4.824895,0.013111$ $-0.018779,4.824878,0.000894$ $-0.051178,4.824643,-0.011472$ $-0.083514,4.824192,-0.023972$ $-0.115794,4.823525,-0.036583$ $-0.148017,4.822643,-0.049298$ $-0.180215,4.821548,-0.062136$ $-0.212295,4.820242,-0.075062$ $-0.244227,4.818729,-0.087970$ $-0.276112,4.817008,-0.100867$ $-0.308137,4.815065,-0.113541$
$-0.307877,4.815082,-0.113960$ $-0.275279,4.817055,-0.102088$ $-0.242562,4.818813,-0.090384$ $-0.209885,4.820347,-0.078711$ $-0.177316,4.821655,-0.067161$ $-0.144800,4.822741,-0.055701$ $-0.112270,4.823608,-0.044225$ $-0.079767,4.824255,-0.032723$ $-0.047297,4.824683,-0.021175$ $-0.014865,4.824892,-0.009559$ $0.017520,4.824883,0.002161$ $0.049858,4.824657,0.014006$ $0.082172,4.824215,0.025924$ $0.114457,4.823557,0.037941$ $0.146723,4.822683,0.050040$ $0.178973,4.821594,0.062230$ $0.211208,4.820289,0.074524$ $0.243429,4.818770,0.086961$ $0.275643,4.817034,0.099631$ $0.308007,4.815073,0.113751$ Curve

$0.280976,4.878307,0.102547$ $0.251285,4.879927,0.092762$ $0.221574,4.881366,0.082382$ $0.191880,4.882623,0.071883$ $0.162200,4.883699,0.061308$ $0.132532,4.884595,0.050675$ $0.102877,4.885309,0.039993$ $0.073235,4.885843,0.029265$ $0.043608,4.886198,0.018487$ $0.013995,4.886372,0.007669$ $-0.015596,4.886367,-0.003210$ $-0.045165,4.886183,-0.014138$ $-0.074715,4.885821,-0.025109$ $-0.104247,4.885280,-0.036114$ $-0.133761,4.884561,-0.047150$ $-0.163269,4.883664,-0.058223$ $-0.192739,4.882590,-0.069316$ $-0.222162,4.881339,-0.080397$ $-0.251568,4.879912,-0.091472$ $-0.281021,4.878305,-0.102474$ $-0.280932,4.878310,-0.102619$ $-0.251282,4.879927,-0.091895$ $-0.221589,4.881365,-0.081233$ $-0.191912,4.882622,-0.070580$ $-0.162274,4.883697,-0.059963$ $-0.132658,4.884591,-0.049367$ $-0.103039,4.885306,-0.038759$ $-0.073432,4.885840,-0.028138$ $-0.043837,4.886196,-0.017496$ $-0.014257,4.886371,-0.006827$ $0.015306,4.886368,0.003880$ $0.044852,4.886186,0.014634$ $0.074388,4.885826,0.025417$ $0.103914,4.885287,0.036236$ $0.133432,4.884570,0.047088$ $0.162942,4.883675,0.057975$ $0.192447,4.882601,0.068903$ $0.221944,4.881349,0.079885$ $0.251437,4.879919,0.090956$ $0.280976,4.878307,0.102547$ Curve

$0.266531,4.910147,0.096702$ $0.238466,4.911590,0.086623$ $0.210397,4.912872,0.076483$ $0.182330,4.913994,0.066332$ $0.154264,4.914955,0.056173$ $0.126200,4.915756,0.046009$ $0.098136,4.916396,0.035840$ $0.070074,4.916876,0.025666$ $0.042013,4.917196,0.015488$
$0.013953,4.917356,0.005306$ $-0.014104,4.917355,-0.004883$ $-0.042159,4.917195,-0.015076$ $-0.070213,4.916874,-0.025273$ $-0.098264,4.916394,-0.035473$ $-0.126314,4.915753,-0.045676$ $-0.154364,4.914952,-0.055882$ $-0.182410,4.913991,-0.066089$ $-0.210451,4.912870,-0.076293$ $-0.238491,4.911589,-0.086497$ $-0.266536,4.910147,-0.096694$ $-0.266526,4.910147,-0.096709$ $-0.238461,4.911590,-0.086543$ $-0.210391,4.912873,-0.076383$ $-0.182322,4.913994,-0.066224$ $-0.154258,4.914955,-0.056068$ $-0.126197,4.915756,-0.045912$ $-0.098136,4.916396,-0.035755$ $-0.070077,4.916876,-0.025596$ $-0.042019,4.917196,-0.015434$ $-0.013962,4.917356,-0.005268$ $0.014092,4.917355,0.004902$ $0.042145,4.917195,0.015077$ $0.070196,4.916874,0.025256$ $0.098246,4.916394,0.035439$ $0.126295,4.915753,0.045627$ $0.154343,4.914953,0.055818$ $0.182390,4.913992,0.066014$ $0.210436,4.912871,0.076217$ $0.238481,4.911589,0.086430$ $0.266531,4.910147,0.096702$ SelNone

Sellast

-Patch

USpans $=10$

VSpans $=10$

Stiffness $=0.0001$

AutomaticTrim=Yes

enter

SelNone

SelCrv

-Loft Tight RebuildCount $=10$ enter

enter

SelNone

Selcrv

Delete

SelAll

Join

Dir

SelLast

Flip

enter

SelLast

Rotate

Copy $=$ Yes

$0,0,0$

120.000000

240.000000

enter

SelNone

Circle

$0,0,0$

0.988136

SelNone

SelLast

ExtrudeCrv BothSides=Yes

Cap=Yes DeleteInput=Yes

2.425920

SelNone

BooleanUnion 
SelAll

enter

-Mesh

SelAll

enter

enter

Weld

SelLast

enter

180

enter

UnifyMeshNormals

SelLast

enter

SelNakedMeshEdgePt

SelLast

enter

Zoom All Extents

enter 